\title{
A NEW CHECKLIST OF ORCHID SPECIES FROM CUBA*
}

\author{
ERnesto Múuica ${ }^{1,3} \&$ Elaine GonZÁlez ${ }^{2}$ \\ ${ }^{1}$ Centro de Investigaciones y Servicios Ambientales ECOVIDA. Carretera a Luis Lazo, $\mathrm{km} 2.5$, \\ Pinar del Río. C.P. 20200, Cuba \\ ${ }^{2}$ Jardín Botánico Orquideario Soroa. Universidad de Pinar del Río. Carretera a Soroa, km 8, Candelaria, \\ Artemisa, Cuba \\ ${ }^{3}$ Author for correspondence:egh75@upr.edu.cu
}

\begin{abstract}
AвSTRACT. In 2014, James D. Ackerman and collaborators published the last checklist of Cuban orchid species in the book, "Orchid Flora of the Greater Antilles". The aim of our study was to determine the current number of orchid species in Cuba and adjacent islands culminating in an updated checklist. We consulted numerous publications and reports, visited four herbaria, and refer to ongoing collaboration with leading specialists. A total of 91 genera and 312 species are documented. We provide comments on taxonomic status on noteworthy species.
\end{abstract}

KEY WORDS: taxonomy, checklist, orchids, Cuba

Introduction. Since the $18^{\text {th }}$ century, the orchids of Cuba have been in the crosshairs of scholars given the island's unique geographic position and large size among the Antilles, and the fact that Cuba became a major regional trade and communications center. Already in 1799 Baltasar Boldo and José Estévez in their Cubensis Prima Flora had described 10 species of Cuban orchids. This Flora remained unpublished in Madrid until 1990. In 1835, another orchid species was described from Cuba, Oncidium lemonianum Lindl., [=Tolumnia lemoniana (Lind1.) Braem]. Following its description, the species was then exported by Captain Sutton and subsequently cultivated by Charles Lemon at Carclew, England. The work by other specialists ensued leading to Ramon de La Sagra's "Historia Física, Politica y Natural de la Isla de Cuba" published in volume XI, between 1850 and 1852, recognized 51 Cuban orchid species. The chapter on orchids in this work was a contribution of Achille Richard. By the end of that century, a total of 188 orchid species had been described from Cuba.

Studies in the 20th century revealed additional orchid taxa in Cuba. In 1938, Julián Acuña Galé published "Catálogo Descriptivo de las orquídeas cubanas" reporting 278 species of Orchidaceae. Later, in 1946, the Frenchman Joseph Silvestre Sauget
(Hermano León) published within "Flora de Cuba" (volume 1) a new expose with few changes compared to earlier catalogues. After an updated treatment in 1951 by Alex D. Hawkes, little new information was reported for the next 33 years. Finally in 1984, a new checklist was published based on the studies conducted by Dr. Helga Dietrich of the University of Jena in Germany, which recorded 88 genera and 300 species. During the final years of the 1980s and the decade of the 1990's, important reports and contributions were made regarding new species by Marta A. Díaz Dumas, James D. Ackerman, Robert Dressler, Carlyle Luer and Juan Llamacho Olmos. In late 1998, after intensive work, a list of documented orchid genera and species in Cuba was submitted by a group of authors from the Orquideario de Soroa, Cuba, which was published in early 2000 as part of the book "Los Géneros de Orquideas Cubanas". The work of Mark A. Nir, "Orchidaceae Antillanae", was also published in the same year.

In the current century, other specialists continued this line of work including the German Hagen Stenzel, the Italo-Costa Rican Franco Pupulin and the Cuban Juan Olmos Llamacho, the latter having authored "The Orchids of Cuba", wherein 305 species are reported within the island. Similarly, an excellent contribution to the knowledge of the orchid flora of

* This text was prepared with substantial contributions by James D. Ackerman, Eric Hágsater, Alec M.Pridgeon, Franco Pupulin, Gerardo A. Salazar and Lawrence W. Zettler. 
Cuba and the Caribbean was advanced by James D. Ackerman and collaborators "Orchid Flora of the Greater Antilles", published in 2014, where 315 species are recorded for the island.

The objective of this study is to present a new checklist of Cuban orchids, taking into account the newest reports, trends and results of current taxonomic studies conducted by leading Cuban national and foreign specialists who for years have devoted their efforts to this task.

Materials and methods. For this study we followed the classification system of Chase et al. (2015). Each species is presented with its basionym and type. For taxa with multiple synonyms, only those whose types are derived from islands near Cuba or Florida are cited. The authors acknowledge that there was no opportunity to examine types in foreign herbaria, for which they trust in those cited by authors with vast experience in the study of the orchid flora within Cuba.

With regard to Cuban herbaria, the authors reviewed the specimens deposited in the Herbarium of the Botanical Garden of Pinar del Río (HUJB), Herbarium Johannes Bisse (HAJB) of the National Botanical Garden, Herbarium of the Universidad Central de Las Villas (HUCLV), and the Herbarium of the Institute of Ecology and Systematics (HAC) that contains the collections of the old Herbarium Sauvalle, Herbarium of the Agronomic Station of Santiago de las Vegas, and those of the Herbarium of the Colegio La Salle. We have also reviewed the specimens deposited in the Herbarium of the Botanical Garden Orquideario Soroa (HOS). When possible, all species consultations are cited in order to facilitate their location to those interested in further research and to obtain direct local consultations in Cuba if necessary.

For those species reported from the island of which the authors have seen no records in the reviewed herbaria, or for those analyzed which have yielded doubts about their true identity, we have relied on information gathered from authors and foreign herbaria which we have not been able to visit. The only exceptions made was regarding those species of which we have not been able to obtain information from national or foreign herbaria, but which have been or are currently being grown at the Botanical Garden Orquideario Soroa, each being duly verified by the authors after seeing the garden's specimens, photographs and drawings there of which are noted in the listing.

As will be noted, we have used data and reports that appear in both historic as well as recent bibliographies, with the aim of arriving at criteria that correspond as closely as possible to the trends observed today in the field of orchidology in the region. In all instances the authors have sought to designate species according to the most recent nomenclature (Chase et. al. 2015). Comments are also made in cases of nomenclatural changes, and for the additions or elimination of genera and species recorded for the country. Endemic species for Cuba are indicated with asterisk $(*)$.

Results and discussion. In comparison to the most recent list published by Ackerman (2014) and following the system of classification proposed by Chase and collaborators (2015), 42 nomenclatural changes have been made, almost all of which have to do with segregations from Pleurothallis towards other genera. One species is synonymized (Encyclia brevifolia $=$ Encyclia pyriformis), six are excluded from the Island of Cuba (see excluded species) and three new species and three natural hybrids have been added (Bletia $\times$ ekmanii, Broughtonia $\times$ guanahacabibensis, Encyclia $\times$ camagueyensis, Encyclia sabanensis, Encyclia viñalensis and Lepanthes caluffii). Only in one case we don't follow Chase et al. (2015) because we prefer to move Pteroglossapis ecristata to the genus Orthochilus and not to Eulophia.

This study has resulted in 91 genera and 312 species for the flora of Cuba. A total of 97 species are endemic, representing $31 \%$ of those registered. The best represented genera are Lepanthes with 28 especies, Epidendrum 26, Encyclia 24, Specklinia 15, Tolumnia and Pleurothallis 10, Habenaria and Vanilla with 9. Finally, the authors hope that the present work will serve as an up-to-date reference for those who engage in the daily struggle to study and conserve species of this family of plants in the country and the Caribbean region as a whole. 


\section{CHECKLIST}

\section{ACinnthera Scheidw.}

Acianthera angustifolia (Lindl.) Luer, Monogr. Syst. Bot. Missouri Bot. Gard. 112: 118. 2007.

Basionym: Pleurothallis angustifolia Lindl., Edward's Bot. Reg. 21: sub. t. 1797. 1835.

Type: Mexico: Jalapa, Deppe s.n. (holotype K).

Pleurothallis confusa Fawc. \& Rendle, J. Bot. 47: 129. 1909.

Type: Jamaica: Bethebara, Wilson \& Wullschlägel 1079 (lectotype, Fawcett \& Rendle, 1909, p. 129, K-L).

Pleurothallis wilsonii Lindl., Ann. Mag. Nat. Hist., ser. 3, 1: 326. 1858; Humboltia angustifolia (Lindl.) Kuntze, Revis. Gen. P1. 2: 667. 1891.

Humboltia wilsonii (Lindl.) Kuntze, Revis. Gen. Pl. 2: 667. 1891.

Acianthera wilsonii (Lindl.) Pridgeon \& M.W.Chase, Lindleyana 16: 247. 2001.

Type: Cuba: Oriente, 1856-57, C.Wright 668 (lectotype K-L).

SPeCIMENS STUdIED: HAJB!: Bisse et al. 45193; HAC!: Alain et al. 7466; HOS! : Pérez \& Bocourt 101; flowers in spirit: Pérez \& Bocourt 088.

Acianthera bissei (Luer) Luer, Monogr. Syst. Bot. Missouri Bot. Gard. 95: 253. 2004.*

BAsionym: Pleurothallis bissei Luer, Lindleyana 14: 108. 1999.

Type: Cuba: Prov. Holguín, Mayarí Abajo, Sierra de Nipe, Loma de la Mensura, J. Bisse et al. HFC 35789 (holotype HAJB!).

Specimen STUdied: HAJB!: Berazain et al. 63388.

Acianthera murex (Rchb.f.) Luer, Monogr. Syst. Bot. Missouri Bot. Gard. 115: 257. 2009.*

BASIONYM: Pleurothallis murex Rchb.f., Flora 48: 276. 1865.

Specklinia murex (Rchb.f.) Luer, Monogr. Syst. Bot. Missouri Bot. Gard. 95: 263. 2004.

Type: Cuba: Prov. Guantánamo, near Monte Verde, 1860-1864, C. Wright 3348 (holotype $\mathrm{K})$.

Pleurothallis trigonifolia Wright ex Lindl., according to Cogn., Fl. Cuba 288. 1866.

Type: Cuba: Guantánamo, near Monte Verde, $C$. Wright s.n. (holotype K).
Pleurothallis papulifolia Luer, Lindleyana 14: 116. 1999.

Acianthera papulifolia (Luer) Luer, Monogr. Syst. Bot. Missouri Bot. Gard. 95: 254. 2004.

Type: Cuba: Holguín: Moa, between Alto de la Calinga y El Toldo, C. Paufet \& I. Silva s.n. (holotype HAJB!).

Specimens STUdied: HAC!: Alain 3811; HUJB!: Urquiola et al. 6310.

Acianthera odontotepala (Rchb.f.) Luer, Monogr. Syst. Bot. Missouri Bot. Gard. 95: 254. 2004.

Basionym: Pleurothallis odontotepala Rchb.f., Flora 48: 275. 1865.

Acianthera odontotepala (Rchb.f.) Luer, Monogr. Syst. Bot. Missouri Bot. Gard. 95: 254. 2004.

Type: Cuba: Oriente: 1860-1864, C. Wright 3349 (holotype K).

Pleurothallis brachypetala Griseb., Cat. Pl. Cub. 257. 1866.

Type: Cuba: "721. Cuba occ.” 1863, Wright 3349b (holotype GOET).

Specimens STUdied: HAJB!: Alvarez et al. 64894; HAC!: Acuña 22991.

Acianthera stenzelii Luer, Mem. New York Bot. Gard. (Orchid Fl. Greater Antilles) 109: 575. 2014.*

Type: Cuba: prov. Sancti Spiritus, Municipio Yaguajay, Martínez-Falcón $s / n$. (Holotype HAC 41200).

Acianthera rubroviridis (Lindl.) Pridgeon \& M.W.Chase, Lindleyana 16: 246. 2001.

Basionym: Pleurothallis rubroviridis Lindl., Ann. Mag. Nat. Hist., ser. 3, 1: 327. 1858.

Humboltia rubroviridis (Lindl.) Kuntze, Revis. Gen P1. 2: 668. 1891.

Type: Cuba: Oriente, C. Wright s.n. (holotype K-L).

Pleurothallis cubensis Lindl., Ann. Mag. Nat. Hist., ser. 3, 1: 328. 1858.

Humboltia cubensis (Lindl.) Kuntze, Revis. Gen. P1. 2: 667. 1891.

Acianthera cubensis (Lindl.) Pridgeon \& M.W.Chase, Lindleyana 16: 243. 2001.

Type: Cuba: Guantánamo, near Monte Verde, $C$. Wright 653 (holotype K-L). 
SPecimens StUdied: HAJB!: Bisse et al. 14686; HOS!: Pérez \& Bocourt 108; flowers in spirit: Pérez \& Bocourt 018.

Acianthera testifolia (Sw.) Solano, Acta Bot. Mex. 97: 50. 2011.

Basionym: Epidendrum testifolium Sw., Prodr. Veg. Ind. Occ. 122. 1788.

Cymbidium testifolium (Sw.) Sw., Nova Acta Regiae Soc. Sci. Upsal. 71. 1799.

Pleurothallis testifolia (Sw.) Lindl., Ann. Mag. Nat. Hist., ser. 3(1): 328. 1858.

Humboltia testifolia (Sw.) Kuntze, Revis. Gen. Pl. 2: 668. 1891.

Apoda-prorepentia testifolia (Sw.) Luer, Monogr. Syst. Bot. Missouri Bot. Gard. 95: 255. 2004.

Type: Jamaica: O. Swartz s.n. (lectotype S, Stenzel, 2007 , p. 80).

Specimens StUdied: HAJB!: Bisse et al. 62259; HOS!: collector unknown 107; flowers in spirit: collector unknown 006.

\section{Anathallis Barbosa Rodrigues}

Anathallis obovata (Lindl.) Pridgeon \& M.W.Chase, Lindleyana 16: 250. 2001.

Basionym: Pleurothallis obovata (Lindl.) Lindl., Edward's Bot. Reg. 28 (Misc.): 75. 1842.

Specklinia obovata Lindl., Edward's Bot. Reg. 25 (Misc.): 137. 1839.

Humboltia obovata (Lindl.) Kuntze, Revis. Gen. Pl. 2: 668. 1891.

Type: Brazil: without collection data (lectotype [Luer, 1999, p. 115] K-L).

Pleurothallis albida Lindl., in Ann Mag. Nat. Hist., ser. 3,1: 327.1858.

Type: Cuba: Monteverde, Wright 655 (K; NY).

SPECIMENS STUDIED: HAJB!: Gutiérrez et al. 67987; HAC!: Casas 17432; HOS!: collector unknown 232; flowers in spirit: collector unknown 143.

Anathallis sertularioides (Sw.) Pridgeon \& M.W.Chase, Lindleyana 16: 250; 2001.

Basionym: Epidendrum sertularioides Sw., Prodr. Veg. Ind. Occ. 122. 1788.

Dendrobium sertularioides (Sw.) Sw., Nova Acta Regiae Soc. Sci. Upsal. 6: 83. 1799.

Pleurothallis sertularioides (Sw.) Spreng., Syst. Veg., ed. 16, 3: 721. 1826.

Specklinia sertularioides (Sw.) Lindl., Gen. Sp.
Orchid. P1. 8. 1830.

Humboltia sertularioides (Sw.) Kuntze, Revis. Gen. Pl. 2: 668. 1891.

Panmorphia sertularioides (Sw.) Luer, Monogr. Syst. Bot. Missouri Bot. Gard. 105: 174. 2006. Type: Jamaica: O. Swartz (lectotype BM 82293). SPECIMENS STUdied: HUJB!: Luis et al. 1937; HOS!: Bocourt et al. 149; flowers in spirit: collector unknown 028.

Anathallis cf. yucatanensis (Ames \& C.Schweinf.) Solano \& Soto Arenas, Icon. Orchid. 5-6: 10. 2002 (publ. 2003).

Basionym: Pleurothallis yucatanensis Ames \& C.Schweinf., Bot. Mus. Leafl. 1(2): 4. 1932.

Specklinia yucatanensis (Ames \& C.Schwienf.) ridgeon \& M.W.Chase, Lindleyana 16: 260. 2001.

Type: Mexico: Yucatan, Campeche, Tuxpeña, 8

Nov 1931, C. L. Lundell 912 (holotype AMES). SPECIMENS STUDIED: We did not find any specimens in the revised Cuban herbaria. Reported by Ackerman (2014) for Peninsula de Guanahacabibes, Pinar del Río province. Epiphytic in dry sclerophyllous forests over limestone. These small, shortly repent plants may be related to Anathallis yucatanensis. The Cuban plants and P. caymanensis are unusual in that they occur in hot, relatively dry, lowland forests. Ackerman suspect (pers. comm. 2010) that the Cuban plants represent an undescribed species but we await better material for positive identification.

\section{Barbosella Schlechter}

Barbosella prorepens (Rchb.f.) Schltr., Repert. Spec. Nov. Regni Veg. 15: 263. 1918.

Basionym: Restrepia prorepens Rchb.f., Gard. Chron., new series 7: 810. 1877.

Pleurothallis prorepens (Rchb.f.) Ames, F.T.Hubb. \& C.Schweinf., Bot. Mus. Leafl. 3: 39. 1934. Type: Costa Rica, Endres 2618 (holotype W).

Pleurothallis dussii Cogn., in Urban, Symb. Antill. 6: 413. 1909.

Barbosella dussii (Cogn.) Dod, Moscosoa 4: 192. 1986.

Type: Guadaloupe, Grande Decouverte d'chemin des Bains á la Savane a Mulet, 1893, R. A. Duss 3339 (holotype, BR). 
Specimens studied: The specimen at K: Wright 7.2.11 is the only one record for Cuba (Loma del Gato), reported by Stenzel \& Llamacho (2002).

Hagen Stenzel (2007) treats Barbosella dussii as a separate species. However, Luer and Ackerman both treat $B$. dussii as a synonym of $B$. prorepens and Pridgeon agrees too. It is widespread throughout the Antilles, Central America and South America (Pridgeon, pers. comm. 2015).

\section{Basiphyllaea Schlechter}

Basiphyllaea carabiaiana (L.O.Williams) Sosa \& M.A.Díaz, Harvard Pap. Bot. 5: 488. 2001.*

BASIONYM: Bletia carabiaiana L.O.Williams, Caldasia 5: 14. 1942.

Type: Cuba: Holguín, Cerro del Fraile, Ekman 3286 (holotype NY).

Specimen Studied: HAC!: León et al. 22472.

Basiphyllaea corallicola (Small) Ames, Schedul. Orchid. 7: 1. 1924.

Basionym: Carteria corallicola Small, Torreya 10: 188. 1910.

Type: United States of America: Florida, Everglade Keys, Smal et al. (holotype NY).

Basiphyllaea angustifolia Schltr., Repert. Spec.

Nov. Regni Veg. 21: 338. 1925.

Type: Cuba: Ekman 17166 (holotype S).

SPECIMEN STUdied: HAJB!: Bisse et al. 35000.

Basiphyllaea hoffmannii M.A.Díaz \& Llamacho, Harvard Pap. Bot. 5: 487. 2001.*

Type: Cuba: Holguín, Moa, Monte La Breña, $J$. A. Llamacho \& A. Chaviano s.n. (holotype HAC!: Acuña 13035).

Basiphyllaea sarcophylla (Rchb.f.) Schltr., Repert. Spec. Nov. Regni Veg. 17: 78. 1921.*

BAsionym: Bletia sarcophylla Rchb.f., Flora 48: 278. 1865.

Tetramicra sarcophylla (Rchb.f.) Cogn., in I.Urban, Symb. Antill. 6: 552. 1910.

Type: Cuba: Pinar del Río, C. Wright 3315 (holotype K).

Specimens Studied: HAJB!: Bisse 19824; HAC!: Alvarez 72258; HAC!: León y Roca 8023; HOS!: Bocourt et al. 222; flowers in spirit: collector unknown 115.

Basiphyllaea volubilis (M.A.Díaz) Sosa \& M.A.Díaz,
Harvard Pap. Bot. 5: 488. 2001.*

BAsionym: Bletia volubilis M.A.Díaz, Revista Jard. Bot. Nac. Univ. Habana 9(2): 32.1988 [publ. 1989].

Type: Cuba: Prov. Holguín: Loma de la Mensura, Bisse et al. (holotype HAJB!).

Basiphyllaea wrightii (Acuña) Nir, Orchid. Antill. 35. 2000. *

BAsionym: Bletia wrightii Acuña, Bol. Estación Exp. Agron. Santiago de las Vegas 60: 157. 1938 [1939].

Type: Cuba: Prov. Pinar del Río, Bahía Honda, $C$. Wright 3316 (holotype GOET).

Specimens studied: HAJB!: Padilla et al. 69301; HOS!: Bocourt \& Mújica 116.

\section{BLetia Ruíz \& Pavón}

Bletia antillana M.A.Díaz \& Sosa, Brittonia 49: 80. 1997.*

Type: Cuba: Prov. Holguín, Cerro Miraflores, López Figueiras 1244 (holotype HAJB!).

SpeCimens StUdied: HUJB!: Urquiola et al. 6353;

HOS!: flowers in spirit: collector unknown 123.

Bletia × ekmanii Serguera \& Sánchez Los., Willdenovia 41: 107-111. 2011.

Type: Cuba: Guantánamo Province, Yateras, Loma "El Cilindro", M. Serguera \& M. Sánchez s.n. (holotype BSC 6430; isotype: B).

Bletia florida (Salisb.) R.Br., in W.T.Aiton, Hortus Kew. ed. 2, 5: 206. 1813.

Basionym: Limodorum floridum Salisb., Prodr. Stirp. Chap. Allerton 9. 1796.

Type: Jamaica: without specific location, (holotype $\mathrm{K})$.

Specimen studied: HUJB!: Urquiola et al. 6449.

Bletia patula Graham, Edinburgh New Philos. 21: 155. 1836.

Type: Haiti: collected by Dr. Fischer and cultivated at the Royal Botanic Garden Edinburgh (holotype FI-W photograph).

Bletia patula var. alba A.D.Hawkes, Phytologia 3: 260. 1950.

Type: United States of America: South Florida, between Black Creek and Gould, Woodbury s.n. (holotype FTG).

SPecimens StUdied: HAJB!: Genes et al. 58342; HOS!: flowers in spirit: Pérez \& Bocourt 022. 
Bletia purpurea (Lam.) A.Dc., Rapp. (Not.) Pl. Rar. Géneve 8: 23. 1840.

BASIONYM: Limodorum purpureum Lam., Encycl. 3: 515. 1791.

Type: Antilles: cultivated at Jardin du Roi, Paris (holotype P).

Bletia verecunda (Salisb.) R.Br., in W.T.Aiton, Hortus Kew. ed. 2. 5: 206. 1813.

Type: Original illustration of Salisbury, based on a plant collected by Catesby from Jamaica (holotype K).

Bletia acutipetala Hook., Bot. Mag. 61: t. 3217. 1833.

Type: USA: South Carolina (holotype K).

Bletia havanensis Lindl., Edward's Bot. Reg. 24 (Misc.): 28. 1838.

Type: Illustration by Mr. Booth based in a plant from Havana collected by Captain Sutton and cultivated by C. Lemon (holotype K).

Specimens studied: HUJB!: Urquiola et al. 557; HOS!: Elaine et al. 248; flowers in spirit: Bocourt \& Martínez 008.

\section{Brachionidium Lindley}

Brachionidium parvum Cogn., Repert. Spec. Nov. Regni Veg. 6: 307. 1909.

Synonyms: Brachionidium sherringii var. parvum (Cogn.) Stehlé, Fl. Descr. Antilles 1: 219. 1939.

Type: Grenada: inter Soulier and Azimar, W. E. Broadway 268 (lectotype K).

Brachionidium dussii Cogn., in I.Urban, Symb. Antill. 6: 451. 1910.

Type: Guadeloupe: Bains-Jaunes, pied de la Soufrière, P. A. Duss 4149 (Holotype B, destroyed); Guadeloupe: La Soufriêre chemin des Dames, C. Sastre 7475 (neotype, Sastre, 1991, p. 207).

Specimen StUdied: HAC!: Alain et al. 5610.

\section{Brassia R. Brown}

Brassia cf. caudata (L.) Lindl., Bot. Reg. 10: t. 832. 1824.

Basionym: Epidendrum caudatum L., Syst. Nat., ed. 10, 1246. 1759.

Malaxis caudata (L.) Willd., Sp. Pl. 4: 93. 1805.

Oncidium caudatum (L.) Rchb.f., in W.G.Walpers,
Ann. Bot. Syst. 6: 766. 1863.

Lectotype: Plumier, Plantarum Americanarum t.

177, (McLeish et al. 1995, p. 120).

Brassia caudata var. hieroglyphica Rchb.f., III

Hort. 27: 20. 1881.

Type: Unknown.

Specimens STUdied: HUJB!: Luis et al. 3490, 3490A y 3490-B; HOS!: Pérez et al. 036, flowers in spirit: Mújica \& Bocourt 011.

Only one species of Brassia is reported for Cuba, B. caudata. Some authors have reported B. lawrenceana Lindl. (Acuña, 1938; Hno. León, 1946), but this is unlikely as the species is a native of northern South America and should therefore also be expected in the Lesser Antilles, Puerto Rico, Hispaniola and Jamaica, the most probable route the species would have followed to reach Cuba. Nir (2000) excludes this species from our area, adducing that A. Richard's report has not been proven so far, since evidences in the reviewed herbaria have not been found. Also $B$. maculata has been reported for Cuba. Acuña (1938) and León (1946) are guided in their report by Fawcett \& Rendle (1910), who cite this species for our country in "Flora of Jamaica". Gloudon \& Tobisch (1995) also mention it for Cuba, apparently led by the same reference. On the other hand, Nir (2000) reports it for Jamaica and Central America. Manuel Caluff (pers. comm. 2008) mentions that he observed a "strange species of Brassia" in Salto de Caburní, Topes de Collantes, Sancti Spíritus province, with flowers having purple sport, very different to $B$. caudata. There may indeed be a second species of Brassia in Cuba.

\section{Broughtonia R. Brown}

Broughtonia cubensis (Lindl.) Cogn., in I.Urban, Symb. Antill. 6: 542. 1910.*

Basionym: Epidendrum cubense Lindl., Edward's Bot. Reg. 29 (Misc.): 17. 1843.

Laeliopsis cubensis (Lindl.) Lindl., Paxton's Fl. Gard. 3: 156. 1853.

Cattleyopsis cubensis (Lindl.) Sauleda \& Adams, Rhodora 86 (848): 455.1984.

Type: Cuba: without specific location, Loddiges 1210 (holotype K).

Specimens studied: HUJB!: Ferro et al. 4310; HOS!: Pérez \& Padilla 028. 
Broughtonia $\times$ guanahacabibensis Múj.Benítez, E.González \& J.M.Díaz, Lankesteriana 15(3): 183185. 2015.

Type: Cuba: Pinar del Río province: Guanahacabibes peninsula, Mújica \& Elaine 241 (holotype HOS!).

In 2004, while carrying out the annual studies on the spatial and temporal dynamics of $B$. cubensis, the authors found an individual of $B$. ortgiesiana in flower, the first report of this species for Cabo San Antonio, Guanahacabibes peninsula. Years later it continued flowering and in 2007 and 2010 fruits observed on that plant. This success confirms that both species are sympatrics and they can be in flower at the same time in the same site. The study area was visited in January 2015 and the authors observed both species in flower simultaneously. Several individuals with intermediate characters were found on site.

Other SPECimen StUdied: Mújica et al. 179 (HOS!, flowers in spirit).

Broughtonia lindenii (Lindl.) Dressler, Taxon 15: 241. 1966.

Basionym: Laelia lindenii Lindl., Orchid. Linden. 10. 1846.

Laeliopsis lindenii (Lindl.) Lindl., Paxton's Fl. Gard. 3: 156. 1853.

Bletia lindenii (Lindl.) Rchb.f., in W.G.Walpers, Ann. Bot. Syst. 6: 431. 1861.

Cattleyopsis lindenii (Lindl.) Cogn., in I.Urban, Symb. Antill. 6: 544. 1910.

Type: Cuba: without specific location, Linden 1805 (holotype K).

?Cattleyopsis guanensis Acuña, Bol. Est. Exper. Agron. Santiago de las Vegas 60, nom. illeg.

Original material: Cuba: Pinar del Río, Guane, Fors 4837 (HAC).

SPECIMENS STUDIED: HUJB!: Urquiola et al. 488; HOS!: Mújica \& Elaine 208; flowers in spirit: Mújica 093.

The authors have observed morphological differences between the plants and flowers of this species at different locations of the island. In the province of Pinar del Río, there are two populations, clearly different in their floral morphology, so Acuña, could have been right when describing Cattleyopsis guanensis.
Broughtonia ortgiesiana (Rchb.f.) Dressler, Taxon 15: 241. 1966.*

Basionym: Bletia ortgiesiana Rchb.f., Hamb. Gartenz. 420. 1860.

Cattleyopsis ortgiesiana (Rchb.f.) Cogn., in I.Urban, Symb. Antill. 6: 546. 1910.

Type: Locality unknown, cultivated in Botanical Garden in Zurich, E. Ortges s.n. (holotype W). Lectotype: Cuba: Guantánamo, Wright 3313, (BM).

SPECIMENS STUdied: HUJB!: Ferro et al. 4304 y 4304-A; HUCLV!: Castañeda 6069; HOS!: Bocourt 096; flowers in spirit: Mújica et al. 106.

\section{Bulbophyllum Thouars}

Bulbophyllum aristatum (Rchb.f.) Hemsl., Biol. Cent.-Amer. Bot. 3: 213. 1884.

BAsionym: Bolbophyllaria aristata Rchb.f., Beitr. Orch. Centr. Amer. 60. 1866.

Phyllorchis aristata (Rchb.f.) Kuntze, Revis. Gen. P1. 2: 677. 1891.

Type: Cultivated in Hort. Schiller s.n. (holotype W).

SPECIMEN STUdied: HAC!: Alain 6722.

Bulbophyllum pachyrachis (A.Rich.) Griseb., Fl. Brit. W. I. 613. 1864.

BAsionym: Pleurothallis pachyrachis A.Rich., in R.de la Sagra, Hist. Fis. Cuba, Bot. 11: 234. 1850; 12: t. 74.1855.

Bolbophyllaria pachyrachis (A.Rich.) Rchb.f., in W.G.Walpers, Ann. Bot. Syst. 6: 24. 1861.

Type: Cuba: 1836, Sagra s.n. (holotype P).

Specimens STUdied: HUJB!: Ferro et al. 4318, 4318-A y 4318-B; HOS!: collector unknown 228; flowers in spirit: collector unknown 161.

\section{Calanthe R. Brown}

Calanthe calanthoides (A.Rich. \& Galeotti) Hamer \& Garay in F.Hamer, Orquid. El Salvador 1: 90. 1974.

Basionym: Ghiesbreghtia calanthoides A.Rich. \& Galeotti, Ann. Sci. Nat. Bot. ser. 3, 3: 28. Jan 1845. Type: Mexico: Prov. Oaxaca, 1842, M. Ghiesbreght s.n. (holotype P).

Calanthe mexicana Rchb.f., Linnaea 18: 406. 1845.

Type: Mexico: Leibold s.n. (holotype W). 
Calanthe cubensis Linden \& Rchb.f., Bonplandia 4: 322.1856.

Type: Cuba: Santiago de Cuba, Sierra Maestra, Monte Libano, Linden s.n. (holotype W).

Calanthe granatensis Rchb.f., Bonplandia 4: 322. 1856.

Type: Colombia: New Grenada, Purdie s.n. (holotype K).

Specimen studied: HAC!: Acuña \& Roig 20009.

\section{Calopogon R. Brown}

Calopogon tuberosus (L.) Britton, Sterns \& Poggenb., var. simpsonii (Small) Magrath, Sida 13: 371. 1989.

Basionym: Limodorum simpsonii Small, Fl. S.E. U.S. 322. 1903.

Calopogon pulchellus var. simpsonii (Chapman ex Small) Ames, Contr. of the Ames Bot. Lab. 1: 18. 1904.

Type: United States of America: Florida, 1892, J. H. Simpson s.n. (holotype NY).

Specimen studied: HAC!: León et al. 20646; Clemente 630.

\section{Campylocentrum Bentham}

Campylocentrum fasciola (Lindl.) Cogn., in Martius, Fl. Bras. 3(6): 520, t. 106. 1906.

Basionym: Angraecum fasciola Lindl., Edward's Bot. Reg. 26: t. 68. 1840.

Aeranthes fasciola (Lindl.) Rchb.f., in W.G.Walpers, Ann. Bot. Syst. 6: 902. 1864.

Type: Guyana: Demerara, Schomburgk s.n. (holotype K).

Aeranthes filiformis Griseb., Fl. Brit. W. I. 625. 1864.

Dendrophylax filiformis (Griseb.) Benth. ex Fawc., A Prov. List of Flow. Pl. of Jamaica 40. 1898. Type: Jamaica: McNab s.n (lectotype K).

Campylocentrum sullivanii Fawc. \& Rendle, J. Bot. 47: 128. 1909.

Type: Jamaica: Hanover, Belvedere, W. Harris 7523 (lectotype BM).

Specimens studied: HAJB!: Díaz et al. 58846; HOS!: Pérez et al. 082.

Campylocentrum jamaicense (Rchb.f. \& Wullsch.) Benth. ex Fawc., Prov. List P1. Jamaica 40. 1893.

BAsionym: Aeranthes jamaicensis Rchb.f. \&
Wullsch., Ann. Bot. Syst. 6: 901. 1864.

Angraecum jamaicense Rchb.f. \& Wullsch., in W.G.Walpers, Ann. Bot. Syst. 6: 901. 1864.

Type: Jamaica: Fairfield, Wullschaegel s.n. (holotype W).

Campylocentrum barrettiae Fawc. \& Rendle, J. Bot. 47: 127. 1909.

Type: Jamaica: Charlemont near Ewarton, $W$. Harris 6580 (BM).

Specimens studied: HUJB!: Urquiola et al. 6352; HOS!: Mújica 137; flowers in spirit: Félix \& Callao 063.

Previously this species was erroneously identified as C. micranthum. Campylocentrum jamaicense is quite distinct as it has bilobed leaves and smooth, fusiform fruits whereas C. micranthum has acute leaves and ribbed fruits (Ackerman, 2014).

Campylocentrum pachyrrhizum (Rchb.f.) Rolfe, Orchid Rev. 11: 246. 1903.

Basionym: Aeranthes pachyrrhiza Rchb.f., Flora 48: 279. 1865

Type: Cuba: C. Wright 3207 (holotype W).

Aeranthes spathaceus Griseb., Cat. Pl. Cub. 264. 1866.

Type: Cuba: Guantánamo, "Retiro”, 1860-1864, C. Wright 3299 (holotype K).

The authors have not seen any specimens in the reviewed Cuban herbaria, but they know it was collected in flower by Pérez \& Bocourt in November 2001, in the proximities of La Bajada, Guanahacabibes peninsula, Sandino municipality, in Pinar del Río province. The authors saw the plant in cultivation with flowers and also saw another sterile individual in the area mentioned.

Campylocentrum poeppigii (Rchb.f.) Rolfe, Orchid Rev. 11: 246. 1903.

Basionym: Angraecum poeppigii Rchb.f., Linnaea 22: 858. 1849.

Type: Cuba: Sabana de Macurijes, Poeppig s.n. (holotype W).

SPeCIMENS STUdied: HUJB!: Urquiola et al. 1127 y 1127-A; HOS!: Pérez et al. 011.

Catasetum Richard ex Kunth

Catasetum integerrimum Hook., Bot. Mag. 67: t. 3823. 1840. 
Type: Guatemala, Skinner ex Hort. Duke of Bedford (Holotype K).

Specimen Studied: HOS!: flowers in spirit: Pérez et al. 025 .

\section{Cochleanthes Rafinesque}

Cochleanthes flabelliformis (Sw.) R.E.Schult. \& Garay, Bot. Mus. Leafl.18: 324. 1959.

Basionym: Epidendrum flabelliforme Sw., Prodr. Veg. Ind. Occ. 123. 1788.

Cymbidium flabelliforme (Sw.) Sw., Nova Acta

Regiae Soc. Sci. Upsal. 6: 73. 1799.

Zygopetalum flabelliforme (Sw.) Rchb.f., in

W.G.Walpers, Ann. Bot. Syst. 4: 652. 1863.

Warcewiczella flabelliformis (Sw.) Cogn., in

I.Urban, Symb. Antill. 4: 182. 1903.

Type: Jamaica: Swartz s.n. (holotype BM).

Specimens studied: HUJB!: Urquiola et al. 6307, 6307-A y 6307-B; HOS!: Bocourt et al. 155; flowers in spirit: Bocourt et al. 095.

\section{Coelia Lindley}

Coelia triptera (Sm.) G.Don ex Steud., Nomencl. Bot. ed. 2, 1: 394. 1840.

Basionym: Epidendrum tripterum Sm., Icon. Pict. Pl. Rar. t. 14. 1793.

Cymbidium tripterum (Sm.) Sw., J. Bot (Schrader) 1799(2): 214. 1800.

Type: No locality, date or collector, Icones Pictae

Plantarum Rariorum t. 14. 1793 (lectotype, Pridgeon 1978, p. 65).

Coelia baueriana Lindl., Gen. Sp. Orchid. Pl. 36. 1833.

Type: Jamaica: flowered in cultivation (holotype K-L).

SPecimens STUdied: HUJB!: Luis et al. 4062; HOS!: flowers in spirit: collector unknown 174.

Comparettia Poeppig \& Endlicher

Comparettia falcata Poepp. \& Endl., Nov. Gen. Sp. P1.1: 42, t. 73. 1836.

Type: Peru: near Cuchero, between Cassapi and Pampayacu, Poeppig 1646 (holotype W).

Comparettia rosea Lindl., Edward's Bot. Reg. 26 (Misc.): 78. 1840.

Type: Spanish Main, Loddiges s.n. (illustration at K-L).
Comparettia cryptocera Morren, La Belgique Horticole 2: 310, t. 53, f. 1. 1852.

Type: unknown.

Comparettia venezuelana Schltr., Repert. Spec. Nov. Regni Veg. 6: 44. 1919.

Type: Venezuela: Federal District: Caracas, von K.

W. John s.n. (holotype B, destroyed).

Comparettia erecta Schltr., Repert. Spec. Nov.

Regni Veg. 7: 185. 1920.

Type: Colombia: Cauca, M. Madero s.n. (holotype B, destroyed).

Comparettia pulchella Schltr., Repert. Spec. Nov. Regni Veg. 7: 185. 1920.

Type: Colombia: Antioquia, M. Madero s.n. (holotype B, destroyed).

SPecimens STUdied: HAJB!: Bisse et al. 46629; HOS!: Pérez et al. 141.

\section{CORYMBorkis Thouars}

Corymborkis flava (Sw.) Kuntze, Revis. Gen. Pl. 2: 658. 1891.

Basionym: Serapias flava Sw., Prodr. Veg. Ind. Occ. 119. 1788.

Neottia flava (Sw.) Sw., Fl. Ind. Occid. 3: 1417. 1806.

Stenorrhynchos flavum (Sw.) Spreng., Syst. Veg. 3: 710.1826.

Tomotris flava (Sw.) Raf., Fl. Tellur 2: 89. 1837.

Chloidia flava (Sw.) Rchb.f., in W.G.Walpers, Ann. Bot. Syst. 6: 644. 1863.

Corymbis flava (Sw.) Hemsl., Biol. Cent.-Amer., Bot. 3: 297. 1884.

Type: Jamaica, O. Swartz s.n. (UPS).

SPeCimens StUdied: We did not find any specimens in the revised Cuban herbaria.

This species has many times been reported for Cuba by different authors. Ackerman (2014) reported it for Granma (Sierra Maestra), Villa Clara (Alturas de Trinidad) and Pinar del Río (Guaniguanico), where we have not found it. The authors have not seen herbarium specimens from the localities above mentioned. However, in February 2015, the authors saw photos of the flowers and living plants collected by Omar Alomá at Pico San Juan, Cienfuegos province. The plants are in cultivation in the Macradenia Botanical Garden, municipality of Palmira, Cienfuegos province, and in Soroa Orchid Botanical Garden, Artemisa province. 
Corymborkis forcipigera (Rchb.f. \& Warsz.) L.O.Williams, Bot. Mus. Leafl. 12: 237. 1946.

BAsIONym: Macrostylis forcipigera Rchb.f. \& Warsz., Bonplandia 2: 96. 1854; Type: Haiti: W. J. Eyerdam 497 (W).

Corymborkis cubensis Acuña, Boletín Bol. Estación Exp. Agron. Santiago de las Vegas 60: 51. 1939.

Type: Cuba: Guantánamo, El Yunque, J. A. Shafer 8001 (holotype NY).

SPecimens STUdied: HUJB!: Luis et al. 3551; HOS!:

Pérez et al. 079.

\section{Cranichis Swartz}

Cranichis diphylla Sw., Prodr. Veg. Ind. Occ. 120. 1788.

Type: Jamaica, Blue Mountains, Swartz s.n. (BM).

Cranichis monophylla Lindl., Orchid. Linden. 27. 1846.

Type: Venezuela, Mérida, Linden s.n. (holotype $\mathrm{K}-\mathrm{L}$ ).

Cranichis tenuiflora Griseb., Cat. Pl. Cub. 268. 1866.

Type: Cuba: C. Wright 3292 (holotype BR).

Cranichis guatemalensis Schltr., Repert. Spec. Nov. Regni Veg. 2: 129. 1906.

Type: Guatemala, auf Felsen bei Chiacam, H. von Türckheim 1379 (holotype B, destroyed).

Specimen Studied: HAC!: Alain et al. 6454.

Cranichis muscosa Sw., Prodr. Veg. Ind. Occ. 120. 1788.

Type: Jamaica, Swartz s.n., (lectotype BM, designated by Garay \& Sweet, 1974, p. 61).

Specimens StUdied: HUJB!: Luis et al. 3121; HOS!:

Bocourt et al. 152; flowers in spirit: Bocourt et al. 084.

Cranichis ovata Wickstr., Kongl. Vetensk. Acad. Handl. 48: 73. 1827.

Type: Guadeloupe, Forsström s.n. (holotype S).

SPECIMENS Studied: We did not find any specimens in the reviewed Cuban herbaria, but we include it here on the basis of report by Ackerman (2014): Shafer 3235 (NY).

Cranichis ricartii Ackerman, Lindleyana 4: 43. 1989.

Type: Puerto Rico: Mun. Maricao, Maricao Forest Reserve, Ackerman et al. 2418 (holotype SEL). SPECIMENS STUdied: Reported for Campamento San
Benito (¿?), AMES: Shafer 4040, Cuba. We did not find any specimens in the revised Cuban herbaria.

Cranichis tenuis Rchb.f., Flora 48: 274. 1865.

Type: Cuba: Oriente, Jan-Jul 1859, C. Wright 1478 (W).

Cranichis pulla Griseb., Cat. Pl. Cub. 268. 1866; pro syn.

Specimens STUdied: HAJB!: Bisse et al. 30352; HAC!: Alain 6456.

\section{Cyclopogon Presley}

Cyclopogon cranichoides (Griseb.) Schltr., Beih. Bot. Centralbl., 37(2): 387. 1920.

Basionym: Pelexia cranichoides Griseb., Cat. Pl. Cub. 269. 1866.

Sauroglossum cranichoides (Griseb.) Ames, Proc. Biol. Soc. Wash. 17: 117. 1904.

Spiranthes cranichoides (Griseb.) Cogn., in I.Urban, Symb. Antill. 4: 338. 1909.

Beadlea cranichoides (Griseb.) Small, Fl. S.E. U.S. ed. 2, 320. 1913.

Type. Cuba: C. Wright 3293 (holotype GOET).

Specimen STUdied: HOS!: flowers in spirit: Pérez et al. 104.

Cyclopogon elatus (Sw.) Schltr., Repert. Spec. Nov. Regni Veg. 6: 53. 1919.

Basionym: Satyrium elatum Sw., Prodr. Veg. Ind. Occ. 119. 1788.

Neottia elata (Sw.) Sw., Kongl. Vetensk. Acad. Handl. 21: 226. 1800.

Ibidium elatum (Sw.) Salisb., Trans. Hort. Soc. London 1: 291. 1812.

Spiranthes elata (Sw.) Rich., De Orchid. Eur. 37. 1817.

Beadlea elata (Sw.) Small ex Britton, Brooklyn Bot. Gard. Mem. 1: 38. 1918.

Type: Jamaica, Swartz s.n. (lectotype BM).

Specimens studied: HAJB!: Bisse et al. 41673; HOS!: Mújica et al. 183; flowers in spirit: Pérez et al. 101.

Cyclopogon laxiflorus Ekman \& Mansf., Ark. Bot. 22A (8): 11. 1929.

BASIONYM: Spiranthes laxiflora (Ekman \& Mansf.,) Jiménez, Phytologia 8: 326. 1962.

Beadlea laxiflora (Ekman \& Mansf.,) Garay, Bot. Mus. Leafl. 28: 300. 1982. 
Type: Haiti: Massif de la Selle, near Marigot, Morne la Visite, E. L. Ekman H7985 (holotype S).

Specimen STUdied: HAJB!: Bisse et al. 41673.

Cyclopogon miradorensis Schltr., Repert. Spec. Nov. Regni Veg. 21: 332. 1925.

Beadlea miradorensis (Schltr.) Garay \& Dunst., Orchids Venezuela 26. 1979.

Type: Mexico: Veracruz, Mirador, Purpus 92 (holotype B, destroyed).

SPECIMENS STUDiED: We did not find any specimens in the revised Cuban herbaria. Its presence is known from the report of Ackerman (2014): UPRRP: Ackerman et al. 3231.

Cyclopogon obliquus (J.J.Smith) Szlach., Fragm. Flor. et Geobot. 39: 417-438. 1994.

BAsionym: Spiranthes obliqua J.J.Smith, Bull. Dep. de 1'Agric. aux Indes Neerland. 43: 74. 1910. Pelexia obliqua (J.J.Smith) Garay, Bot. Mus. Leafl. Harv. Univ. 28: 345. 1982.

Type: Java: Buitenzorg, J. J. Smith s.n. (holotype L).

Manniella hongkongensis S.Y.Hu \& G.Barretto, Chung Chi J. 13(2): 6. 1976.

Type: China, Hong Kong, S. Y. Hu 13266 (K).

Pelexia hameri Garay, Bot. Mus. Leafl. Harv. Univ. 26(1): 22. 1978.

Type: El Salvador, F. Hamer 613 (AMES).

SPECIMEN STUdied: HAJB: Ackerman et al. 3033.

This species has a complex nomenclatural history, which has been discussed by Garay (1982) and Blanco (2002). DNA sequence data corroborate the placement of this species in Cyclopogon (G. A. Salazar, unpubl. data). It is worthy of note that this species, which is a member of an exclusively Neotropical lineage, has naturalized itself in the Old World tropics (see Cribb and Ormerod 1999; Blanco 2002).

\section{Cyrtopodium R. Brown}

Cyrtopodium punctatum (L.) Lindl., Gen. Sp. Orchid. Pl. 188. 1833.

Basionym: Epidendrum punctatum L., Syst. Nat., ed. 10, 2: 1246. 1759; Type: Plumier s.n. (holotype original illustration at $\mathrm{P}$ ).

Specimens Studied: HUJB!: Urquiola et al. 4341; HOS!: flowers in spirit: Pérez \& Bocourt 001.
Dendrophylax Reichenbach f.

Dendrophylax alcoa Dod, Moscosoa 2: 5. 1983.

Type: Dominican Republic: Prov. Pedernales, Cabo Rojo, Las Abejas, Sierra Baoruco, D. Dod 871 (holotype JBSD).

SPECIMEN STUDIED: HOS!: flowers in spirit: collector unknown 142.

In November 2013, the authors observed individuals of this species in flower in the Soroa Orchid Botanical Garden, erroneously identified as D. barrettiae, collected in the north of Las Tunas province, which agrees with Ackerman's report (2014).

Dendrophylax barrettiae Fawc. \& Rendle, J. Bot. 47: 266. 1909.

Type: Jamaica: near Browns Town, T. MoultonBarrett s.n. (K).

Campylocentrum arizajuliae Ames, Bot. Mus. Leafl. 6: 23. 1938.

Type: Dominican Republic: Prov. La Romana, Ariza Julia s.n. (holotype AMES).

SPECIMEN STUdied: HOS!: Padilla et al. 038.

In November 2013, a joint expedition of the authors and specialists from Flora and Fauna reported a population of more than 200 individuals of this species in the protected area Los Pretiles, Mantua municipality, in Pinar del Río province. Many mature individuals were in flower, which facilitated the identification of this species. Ackerman (2014) reports it for Cuba on the basis of a picture published by Marta A. Díaz (1988) in her book "The Native Orchids of Cuba", and erroneously identified as Polyradicion lindenii (Lindl.) Garay. The specimen that we cite here is the first record of this species for Cuba.

Dendrophylax gracilis (Cogn.) Garay, J. Arnold Arbor. 50: 467. 1969.*

Basionym: Polyrrhiza gracilis Cogn., in I.Urban, Symb. Antill. 6: 679. 1910.

Polyradicion gracilis (Cogn.) H.Dietr., Wiss.

Z. Friedrich-Schiller-Univ. Jena. MathNaturwiss. Reihe 32: 61. 1983.

Type: Cuba: Los Hondones, Valparaiso, C. Wright 3300 (holotype GOET).

Specimen Studied: HAJB!: Bisse et al. 49384.

Dendrophylax lindenii (Lindl.) Benth. ex Rolfe, Gard. Chron., ser. 3, 4: 533. 1888. 
Basionym: Angraecum lindenii Lindl., Gard. Chron. 1846: 135. 1846.

Type: Cuba: Santiago de Cuba, 1844, J. Linden s.n. (holotype K-L).

Aeranthus lindenii (Lindl.) Rchb.f., Ann. Bot. Syst. 6: 902. 1864.

Polyrrhiza lindenii (Lindl.) Cogn. in Urban, Symb. Antill. 6: 680. 1910.

Polyradicion lindenii (Lindl.) Garay, J. Arnold Arbor. 50: 467. 1969.

SPeCiMENS StUdied: HUJB!: Ferro et al. 4305; HOS!: Pérez et al. 024; flowers in spirit: Bocourt et al. 062 .

The differences in the flowering season of this species among the Cuban populations (OctoberDecember) and those from Florida (July) deserve to be studied further.

Dendrophylax monteverdi (Rchb.f.) Ackerman \& Nir, Lankesteriana 4: 53. 2004.

Basionym: Aeranthes monteverdi Rchb.f., Flora 48: 279. 1865.

Campylocentrum monteverdi (Rchb.f.) Rolfe, Orchid Rev. 11: 247. 1903.

Harrisella monteverdi (Rchb.f.) Cogn., in I.Urban, Symb. Antill. 6: 687. 1910.

Type: Cuba: C. Wright 1497 (holotype K).

Epidendrum filiforme Sw., Prodr. Veg. Ind. Occ. 126. 1788.

Limodorum filiforme (Sw.) Sw., Nova Acta Regiae Soc. Sci. Upsal. 6: 80. 1799.

Campylocentrum filiforme (Sw.) Cogn. ex Kuntze, Revis. Gen. Pl. 3(2): 298. 1898.

Harrisella filiformis (Sw.) Cogn., in I.Urban, Symb. Antill. 6: 687. 1910.

Dendrophylax filiformis (Sw.) Carlsward \& Whitten, Int. J. Plant. Sci. 164: 50. 2003, non Dendrophylax filiformis (Griseb.) Benth. ex Fawc.

Type: Hispaniola: Swartz s.n. (BM).

SPecimens STUDied: HAJB!: Alvarez et al. 4554-A; HUJB!: Urquiola 7884.

Dendrophylax porrectus (Rchb.f.) Carlsward \& Whitten, Int. J. P1. Sci. 164: 51. 2003.

BAsIONYM: Aeranthes porrecta Rchb.f., Flora 48: 279. 1865.

Campylocentrum porrectum (Rchb.f.) Rolfe,
Orchid Rev. 11: 247. 1903.

Harrisella porrecta (Rchb.f.) Fawc. \& Rendle, J. Bot. 47: 266. 1909.

Type: Cuba: C. Wright 3302 (holotype K).

Harrisella uniflora H.Dietr., Orchidee 33: 18. 1982.

Type: Cuba: Prov. Granma, Bartolomé Masó, Sierra Maestra, Loma de la Sabina, Bisse et al. (holotype HAJB!).

Specimen studied: HOS!: Mújica 166.

Dendrophylax varius (J.F.Gmel.) Urb., Repert. Spec. Nov. Regni Veg. 15: 306. 1918.

Basionym: Orchis varia J.F.Gmel., Syst. Plant, ed. 13: 53. 1791.

Type: Original Plumier drawing (holotype P).

Limodorum flexuosum Willd., Sp. Pl. 4: 128. 1805.

Dendrophylax flexuosus (Willd.) Urban, Repert.

Spec. Nov. Regni Veg. 15: 108. 1917.

Dendrophylax hymenanthus Rchb.f., in

W.G.Walpers, Ann. Bot. Syst. 6: 903. 1864. Aeranthes hymenantha (Rchb.f.) Griseb., Cat. P1. Cub. 264. 1866.

Type: Cuba: Oriente, 1860, C. Wright 1692 (holotype W).

Specimens studied: HAJB!: Bisse et al. 30859; HOS!: Pérez \& Bocourt 136; flowers in spirit: Pérez \& Bocourt 058.

\section{Dichaea Lindley}

Dichaea glauca (Sw.) Lindl., Gen. Sp. Orchid. Pl. 209. 1833.

Basionym: Epidendrum glaucum Sw., Prodr. Veg. Ind. Occ. 124. 1788.

Cymbidium glaucum (Sw.) Sw., Nova Acta Regiae Soc. Sci. Upsal. 6: 71. 1799.

Epithecia glauca (Sw.) Schltr., Orchis 9: 26. 1915. Dichaeopsis glauca (Sw.) Schltr., Beih. Bot. Centralbl., 36(2): 519. 1918.

Type: Jamaica, Swartz s.n. (isolectotypes G, M, S, UPS, W).

SPeCiMENS StUdied: HAJB!: Bisse et al. 40428; HOS!: Pérez et al. 066.

Dichaea graminoides (Sw.) Lindl., Gen. Sp. Orchid. P1. 209. 1833.

Basionym: Epidendrum graminoides Sw., Prodr. Veg. Ind. Occ. 125. 1788. 
Cymbidium graminoides (Sw.) Sw., Nova Acta Regiae Soc. Sci. Upsal. 6: 71. 1799.

Dichaea graminea Griseb., Fl. Brit. W. I. 625. 1864.

Epithecia graminoides (Sw.) Schltr., Orchideen Beschreib. Kult. Zucht. 534. 1914.

Dichaeopsis graminoides (Sw.) Schltr., Beih. Bot. Centralbl., 36(2): 519. 1918.

Type: Jamaica: Swartz s.n. (BM).

Specimen Studied: HAJB!: Dietrich et al. 67110.

Dichaea hystricina Rchb.f., Flora 48: 279. 1865.

Type: Cuba: Monteverde, C. Wright 1487 (holotype K).

SPECIMENS STUdied: HAJB!: Bisse et al. 44579; HOS!: Pérez et al. 077.

Dichaea latifolia Lindl., Gen. Sp. Orchid. P1. 208. 1833.

Dichaea muricata var. latifolia (Lindl.) Griseb., Fl. Brit. W. I. 624.1864

Type: St. Vincent: Guilding s.n (holotype K, reported by Garay \& Sweet, 1974).

Specimens StUdied: HAJB!: Bisse et al. 40310; HOS!: Pérez et al. 081.

Dichaea morrisii Fawc. \& Rendle, J. Bot. 48: 107. 1910.

Epithecia morrisii (Fawc. \& Rendle) Schltr., Orchis 9: 26. 1915.

Dichaeopsis morrisii (Fawc. \& Rendle) Schltr., in I.Urban, Symb. Antill. 8: 146. 1920.

Type: Jamaica: Mt. Moses, J.P. (Jamaica Plants) 2269, Morris s.n. (holotype BM).

Cymbidium muricatum Sw., Nova Acta Regiae Soc. Sci. Upsal. 6: 71. 1799.

Dichaea muricata (Sw.) Lindl., Gen. Sp. Orchid. Pl. 209. 1833.

Type: Jamaica: Swartz s.n. (lectotype W-R 2529).

SPECIMEN STUdied: HAC!: Hioram 4906.

Dichaea pendula (Aubl.) Cogn., in I.Urban, Symb. Antill. 4: 182. 1903.

Basionym: Limodorum pendulum Aubl., Hist. Pl. Guiane 2: 819, 1775.

Type: French Guiana, "in sylvis Comitatus de Gene", Aublet s.n. (Histoire des Plantes de la Guiane Françoise 2: 819, t. 322. 1775).

Epidendrum echinocarpum Sw., Prodr. Veg. Ind. Occ. 124. 1788.

Cymbidium echinocarpon (Sw.) Sw., Nova Acta
Regiae Soc. Sci. Upsal. 6: 71. 1799.

Pachyphyllum echinocarpon (Sw.) Spreng., Syst. Veg. 3: 731. 1826.

Dichaea echinocarpa (Sw.) Lindl., Gen. Sp. Orchid. P1. 208. 1833.

Type: Jamaica: Swartz s.n. (lectotype designated by Pupulin 2007, p. 113).

Dichaea echinocarpa var. lobata Ames \& Correll,

Bot. Mus. Leafl.11: 71. 1955.

Specimen Studied: HAJB!: Bisse et al. 37581.

Dichaea trichocarpa (Sw.) Lindl., Gen. Sp. Orchid. P1. 209. 1833.

Basionym: Epidendrum trichocarpum Sw., Prodr. Veg. Ind. Occ. 124. 1788.

Cymbidium trichocarpon (Sw.) Sw., Nova Acta Regiae Soc. Sci. Upsal. 6: 71. 1799.

Type: Jamaica, Swartz s.n. (lectotype W-R-25293, designated by Pupulin, 2007, p. 133).

Specimen STUdiEd: HAJB!: Bisse \& Dietrich 46227.

\section{Dilomilis Rafinesque}

Dilomilis bissei H.Dietr., Orchidee 35: 201. 1984.*

Type: Cuba: Prov. Guantánamo: Municipio Baracoa, Santa María, Altiplano de la Mina de Iberia, $700 \mathrm{~m}$, Areces et al. (holotype HAJB 25769!).

Dilomilis elata (Benth.) Summerh., Taxon 10: 253. 1961.

Basionym: Octadesmia elata Benth., in G.Bentham \& J.D.Hooker, Gen. Plant. 3: 525. 1883.

Type: Jamaica: Jamaica Plants 2382, Syme s.n. (holotype K).

Specimen StUdied: HUJB!: Urquiola et al. 6348.

Dilomilis montana (Sw.) Summerh., Taxon 10: 253. 1961.

Basionym: Epidendrum montanum Sw., Prodr. Veg. Ind. Occ. 121. 1788.

Cymbidium montanum (Sw.) Sw., Nova Acta Regiae Soc. Sci. Upsal. 6: 72. 1799.

Bletia montana (Sw.) Rchb.f., in W.G.Walpers, Ann. Bot. Syst. 6: 445. 1862.

Tetramicra montana (Sw.) Griseb., Fl. Brit. W. I. 622.1864.

Octadesmia montana (Sw.) Benth. \& Hook.f., Gen. P1. 3: 525. 1883.

Type: Jamaica: Swartz s.n. (BM).

SPecimen STUdied: HAJB!: Bassler et al. 53408. 
Dilomilis oligophylla (Schltr.) Summerh., Taxon 10: 253. 1961.*

Basionym: Octadesmia oligophylla Schltr., Repert. Spec. Nov. Regni Veg. 21: 336.1925.

Type: Cuba: Sierra de Nipe, Ekman 15275 (holotype S).

Specimen Studied: HAJB!: Bisse et al. 40295.

\section{Dinema Lindley}

Dinema cubincola (Borhidi) H.Dietr., Wiss. Z. Friedrich-Schiller-Univ. Jena. Math-Naturwiss. Reihe 29(4): 524. 1980.*

Basionym: Epidendrum cubincola Borhidi, Acta Bot. Acad. Sci. Hung. 22(1): 295. 1976.

Type: Cuba: Santiago de Cuba, Sierra Maestra, Gran Piedra, Acuña 21104 (holotype originally deposited at SV, now at HAC).

Specimens studied: HAJB!: Bisse et al. 40293; HOS!: Agapito et al. 010; flowers in spirit: collector unknown 086.

\section{Domingoa Schlechter}

Domingoa haematochila (Rchb.f.) Carabia, Mem. Soc. Cub. Hist. Nat. "Felipe Poey" 17: 143. 1943.

BASIONYM: Epidendrum haematochilum Rchb.f., Flora 48: 277. 1865.

Type: Cuba, C. Wright 3338 (holotype K).

Epidendrum hymenodes Rchb.f., Flora 48: 277.

1865, non Lindl., Fol. Orchid. Epidendrum 58. 1853.

Domingoa hymenodes Schltr., in I.Urban, Symb. Antill. 7: 497. 1913.

Type: Cuba: C. Wright 3321 (holotype K).

Epidendrum broughtonioides Griseb., Cat. Pl. Cub. 261. 1866.

Type: Cuba: C. Wright 3321 (MO).

SPecimens STUdied: HUJB!: Luis et al. 3493; HOS!: Mújica \& Elaine 207; flowers in spirit: Pérez et al. 020 .

\section{Elleanthus Presley}

Elleanthus cephalotus Garay \& H.R.Sweet, J. Arnold Arbor. 53: 390. 1972, (avowed substitute for Elleanthus capitatus (R.Br.) Rchb. f., ex Cogn. in Urban, Symb. Antill. 6: 561. 1910; non Elleanthus capitatus (Poepp. \& Endl.) Rchb.f., Walp. Ann. Bot. Syst. 6: 475. 1862).
BASIONYM: Bletia capitata R.Br., in W.T.Aiton, Hort. Kew., ed. 2, 5: 206. 1813.

Type: West Indies (holotype BM).

Specimens studied: HAJB!: Bisse et al. 40555; HOS!: Pérez et al. 043.

Elleanthus cordidactylus Ackerman, Lindleyana 2: 122. 1987.

Type: Puerto Rico: Municipality of Río Grande: Luquillo Mts., Quebrada Grande, S of El Verde, Ackerman 1843 (holotype SEL).

Specimen Studied: HAJB!: Bisse et al. 52445.

\section{Eltroplectris Rafinesque}

Eltroplectris calcarata (Sw.) Garay \& H.R.Sweet, J. Arnold Arbor. 53: 390. 1972.

Basionym: Neottia calcarata Sw., Fl. Ind. Occid. 3: 1413, t. 28. 1806.

Stenorrhynchos calcaratum (Sw.) L.C.Rich., De Orchid. Eur. 37. 1817.

Pelexia calcarata (Sw.) Cogn., in I.Urban, Symb. Antill. 6: 328. 1909.

Centrogenium calcaratum (Sw.) Schltr., Beih. Bot. Centralbl. 37(2): 452. 1920.

Spiranthes calcarata (Sw.) J.Jiménez, Phytologia 8: 326. 1962.

Type: Haiti: Swartz s.n. (lectotype S).

Specimens Studied: HAJB!: Bassler et al. 60545; HOS!: Mújica 175.

\section{ENCYClia Hooker}

Encyclia acutifolia Schltr., in I.Urban, Symb. Antill. 9: 66. 1923.

Epidendrum acutifolium (Schltr.) Carabia, Mem. Soc. Cub. Hist. Nat. "Felipe Poey" 17: 146. 1943.

Type: Cuba: Prov. Holguín, Sierra de Nipe, Río

Piloto, Ekman 3374 (holotype S).

Specimens studied: HAC!: Acuña 13485; HAC!: Álvarez et al. 38214; HOS!: flowers in spirit: Mújica \& Bocourt 180.

Encyclia altissima Schltr., Orchideen Beschreib. Kult. Zücht. 201. 1914.

BASIONym: Epidendrum altissimum Bateman ex Lindl., Edward's Bot. Reg. 24 (Misc.): 38. 1838, non Jacq., 1760, non Lehmann \& F. Kränzl. 1899. Epidendrum hodgeanum A.D.Hawkes, Orquídea 
(Río de Janeiro) 18: 176. 1957.

Encyclia hodgeana (A.D.Hawkes) Beckner, Phytologia 20: 217. 1970.

Type: Bahamas, Skinner ex Hort. Bateman s.n. (holotype K).

SPECIMENS STUDIED: BSC: Martinez \& Fagilde 20631; HOS!: flowers in spirit: collector unknown 121.

Encyclia bipapularis (Rchb.f.) Acuña, Bol. Estación Exp. Agron. Santiago de las Vegas 60: 80. 1939.*

BAsionym: Epidendrum bipapulare Rchb.f., Flora 48: 277. 1865; Type: Cuba: 1860-1864, C. Wright 3328 (holotype K).

Epidendrum tampense var. amesianum Correll, Lloydia 10: 211. 1947.

Type: Cuba: Villa Clara, near San Blas, J. G. Jack s.n. (holotype AMES).

Specimens Studied: HAC!: Clement 3613; HOS!: Mújica 180; flowers in spirit: collector unknown 112.

Although Ackerman (2014) reports this species from the eastern provinces of the island, the authors agree with Llamacho and Larramendi (2005) that this species is restricted to the west of Cuba, specifically to Artemisa province and its limits with Pinar del Río province. The reports for the eastern Cuba are likely confused with another species.

Encyclia bocourtii Múj.Benítez \& Pupulin, Harvard Pap. Bot. 10: 228. 2005.*

Type: Cuba: Pinar del Río, Sandino, Guanahacabibes Peninsula, Guanahacabibes National Park, Cabo San Antonio, road between Faro Federico Roncali and Las Tumbas, near El Francés beach, Pupulin et al. 4127 (holotype HOS!, isotype USJ).

Other SPECIMENS STUdied: Mújica et al. 111 (HOS!); Mújica et al. 014 (HOS!, flowers in spirit); collector unknown 177 (HOS!, flowers in spirit).

Encyclia cajalbanensis Múj.Benítez, Bocourt, \& Pupulin, Lankesteriana 4: 211. 2004.*

Type: Cuba: Pinar del Río, Municipio La Palma, ladera sur de la meseta de Cajálbana, a $2.5 \mathrm{~km}$ después de El Burén, en Sendero Interpretativo, Mil Cumbres, Bocourt et al. 117 (holotype HOS!). Other SPECIMEN STUdied: Mújica \& Bocourt 035 (HOS!, flowers in spirit).
Encyclia × camagueyensis Rodríguez, González, Sauleda, Villalobos \& Esperón. Orchids Digest April, May, June 110-113. 2009.

Type: Cuba: Camagüey: Municipality of Minas, Loc. "Los Orientales", HIPC 10462: Rodríguez Seijó 036.

SPECIMEN STUDIED: HOS!, flowers in spirit: collector unknown 177.

This is a natural hybrid between E. altissima and E. phoenicea, discovered in the north of Camagüey province.

Encyclia fucata (Lindl.) Schltr., Orchideen Beschreib. Kult. Zücht. 209. 1914.

BASIONYM: Epidendrum fucatum Lindl., Edward's Bot. Reg. 24 (Misc.): 15. 1838.

Type: Cuba: Havannah, (holotype K-L).

Epidendrum sagreanum A.Rich., in R.de la Sagra,

Hist. Fis. Cuba Bot. 11: 235, t. 75. 1850.

Type: Cuba: Sagra s.n. (holotype P).

Epidendrum hircinum A.Rich., in R.de la Sagra,

Hist. Fis. Cuba Bot. 11: 236, pl. 77. 1850.

Encyclia hircina (A.Rich.) Acuña, Bol. Estación

Exp. Agron. Santiago de las Vegas 60: 74. 1939.

Type: Cuba: Sagra s.n. (holotype P).

Epidendrum obcordatum Jenn., Annuaire Conserv.

Jard. Bot. Genéve 11: 101. 1917.

Type: Cuba: near Nueva Gerona, 12 May 1910, O.

E. Jenn. 651 (holotype CM).

SPecimens STUdied: HUJB!: Luis et al. 3798; HOS!:

Pérez et al. 008; flowers in spirit: Pérez \& Bocourt 015.

Encyclia gravida (Lindl.) Schltr., Beih. Bot. Centralbl. 36(2): 472. 1918.

BASIONYM: Epidendrum gravidum Lindl., J. Hort. Soc. London 4: 114. 1849.

Epidendrum oncidioides var. gravidum (Lindl.) Ames, F.T.Hubb. \& C.Schweinf., Bot. Mus. Leafl. 3: 104. 1935.

Type: Mexico: near Xapatam (Jalapa), Hartweg s.n. (holotype K-L).

Epidendrum sintenisii Rchb.f., Ber. Deutsch. Bot. Ges. 3: 277. 1885.

Encyclia sintenisii (Rchb.f.) Britton, in Britton \& Wilson, Bot. Porto Rico 5: 197. 1924.

Type: Puerto Rico, Indiera Fria, near Maricao, Sintenis 506 (holotype W). 
Epidendrum monticolum Fawc. \& Rendle, J. Bot. 47: 124. 1909.

Encyclia monticola (Fawc. \& Rendle) Acuña, Bol. Estación Exp. Agron. Santiago de las Vegas 60: 72.1939.

Type: Jamaica: Holly Mount, Mt. Diabolo, Harris 10467 (holotype BM).

Specimens studied: HAJB!: Genes et al. 59344; HOS!: Pérez \& Bocourt 125; flowers in spirit: Pérez \& Bocourt 049.

Encyclia grisebachiana (Cogn.) Acuña, Bol. Estación Exp. Agron. Santiago de las Vegas 60: 73. 1939.*

Basionym: Epidendrum grisebachianum Cogn., in I.Urban, Symb. Antill. 6: 495. 1910.

Prosthechea grisebachiana (Cogn.) W.E.Higgins, Phytologia 82: 378. 1997.

Type: Cuba: 1860-1864, C. Wright 3326 (holotype GOET).

Specimens studied: HUJB!: Novo et al. 1242; HOS!: Pérez et al. 030; flowers in spirit: collector unknown 156.

Encyclia howardii (Ames \& Correll) Hoehne, Arq. Bot. Estado Sao Paulo, nova série, 2: 152. 1952.*

Basionym: Epidendrum howardii Ames \& Correll, Bot. Mus. Leafl. 11: 2. 1943.

Encyclia howardii (Ames \& Correll) H.Dietr., Revista Jard. Bot. Nac. Univ. Habana 5(1): 49. 1984.

Type: Cuba: Oriente: Sierra de Moa, R. A. Howard 5939 (holotype AMES).

Specimen studied: HAJB!: Bisse et al. 9292.

Encyclia isochila (Rchb.f.) Dod, Moscosoa 4: 193. 1986.

BASIONYM: Epidendrum isochilum Rchb.f., Bonplandia 4: 326. 1856.

Type: Dominican Republic, Schiller s.n., (holotype W 44).

Epidendrum isochilum var. tridens Rchb.f., Ber. Deutsch. Bot. Ges. 3: 277. 1885.

Encyclia isochila var. tridens (Rchb.f.) Dod, Moscosoa 4: 193. 1986.

Type: Dominican Republic (holotype W 43).

Epidendrum bletioides Griseb., Fl. Brit. W. I. 615. 1864.

Type: Jamaica: Hanover, Purdie s.n. (holotype K). Epidendrum belvederense Fawc. \& Rendle, J. Bot. 47: 123. 1909.
Encyclia belvederense (Fawc. \& Rendle) H.Dietr., Wiss. Z. Friedrich-Schiller-Univ. Jena. MathNaturwiss. Reihe 33: 710. 1984.

Type: Jamaica: Hannover, Belvedere, W. Harris 7620 (holotype BM).

Epidendrum belvederense var. brevifolium Cogn.,

Feddes Repert. Spec. Nov. Regni Veg. 7: 123. 1909.

Type: Jamaica, near Lancaster, W. Harris 7541

(holotype UCWI).

SPecimen Studied: HAJB!: Bisse et al. 44784.

Encyclia moebusii H.Dietr., Feddes Repert. Spec. Nov. Regni Veg. 96: 563. 1985.*

Type: Cuba: Prov. Guantánamo, Küstenregion zwischen Imias und Maisi, H. Dietrich s.n. (holotype JE).

Specimens studied: HAC!: Alain 3389; HOS!: Pérez et al. 074.

Encyclia monteverdensis M.A.Díaz \& Ackerman, Lankesteriana 4: 50. 2004.*

Type: Cuba: Prov. Guantánamo, Monte Verde, $C$. Wright 1489 (holotype AMES 73736).

SPeCimens STUdied: We did not find any specimens in the revised Cuban herbaria.

Encyclia navarroi Vale \& Rojas, Ann. Bot. Fennici 49: 83-86. 2012.*

Type: Cuba: Pinar del Río, Península de Guanahacabibes, María La Gorda, Holotype: HAC (SV 42604).

Specimen studied: HOS!, flowers in spirit: Pepe 164.

Recently this species has been described for Cabo Corrientes, Guanahacabibes Peninsula, Pinar del Río province. We failed to find other individuals ot this taxon when following the geo-coordinates given by the authors in the protologue. After many years of study on this peninsula, it is our opinion that in the area, a process of natural hybridization is taking place among E. plicata-E. bocourtii and E. phoenicea-E. plicata, species that flower simultaneously in the area and attract the same pollinator. This has given rise to a great number of morphs in the area that we have photographed and documented. Before the publication of $E$. navarroi, we knew about a population of a similar morph outside the limits of Guanahacabibes peninsula in Pinar del Río province. José L. Bocourt, botanical illustrator with vast experience in orchids, showed us a 
picture with the dissection of flowers of E. phoenicea - "E. navarroi?"- E. bocourtii, which shows how this taxon could be a natural hybrid of those species.

Encyclia nematocaulon (A.Rich.) Acuña, Bol. Estación Exp. Agron. Santiago de las Vegas 60: 77. 1939.*

Basionym: Epidendrum nematocaulon A.Rich., in R.de la Sagra, Hist. Fis. Cuba Bot. 11: 238, pl. 79. 1850.

Type: Cuba: flowered in cultivation "Fac. Med. Paris" July 1844 (holotype P).

Specimen Studied: HAC!: Osment 20602.

Encyclia ochrantha (A.Rich.) Withner, Cattleyas \& Relatives 4: 64. 1996.*

BAsionym: Epidendrum ochranthum A.Rich., in R. de la Sagra, Hist. Fis. Cuba Bot. 11: 237, t. 78. 1850.

Type: Cuba: Sagra s.n. (holotype P).

SPeCimens STUdied: We did not find any specimens in the revised Cuban herbaria. The locality of the type is ignored.

Encyclia oxypetala (Lindl.) Schltr., Notizbl. Bot. Gart. Berlin-Dahlem 7: 277.1918.*

Basionym: Epidendrum oxypetalum Lindl., Orch. Lindl. 8. 1846.

Type: Cuba: Guantánamo, J. Linden s.n. (holotype $\mathrm{K}-\mathrm{L}$ ).

SPeCimens STUdied: HAC!: León et al. 6657; HOS!: flowers in spirit: Bocourt 130.

Encyclia phoenicea (Lindl.) Neumann, Rev. Hort. sér. 2, 4: 137. 1845-1846. *

BAsionym: Epidendrum phoeniceum Lindl., Edward's Bot. Reg. 27: (Misc.) 57. 1841.

Type: Cuba: Monte Verde, K-L).

Epidendrum duboisianum (Neumann) Brongn. ex A.Rich., in R.de la Sagra, Hist. Fis. Cuba Bot. 11: 239. 1850.

Type: Cuba: A. Brongniart s.n. (holotype P).

Epidendrum oblongatum A.Rich., in R.de la Sagra, Hist. Fis. Cuba Bot. 11: 239, pl. 80. 1850.

Encyclia oblongata (A.Rich.) Acuña, Bol. Estación Exp. Agron. Santiago de las Vegas 60: 80. 1939.

Type: Cuba: R. de la Sagra s.n. (holotype P).

Encyclia havanense Bello, Esperón \& Sauleda,

New World Orchidaceae - Nomenclatural Notes - Issue No. 4, 2013.
Type: Cuba: Pinar del Río, municipality of La Palma, 4 miles from Cajalbanas, (Holotype: Cuba: Havana, M. Quesnel s.n., P).

Encyclia hamiltonii Sauleda \& Esperón, New World Orchidaceae - Nomenclatural Notes Issue No. 5, 2013.

Type: Ex hort., Sauleda, R. P.\& E. Esperon s.n. (holotype FTG).

SPECIMENS STUDIED: HPPR!: Urquiola 242; HOS!: flowers in spirit: from diferent localities, Pérez \& Bocourt 016, 017, 023 and 024.

Some authors also cite it for Cayman Islands. The description was based on Cuban material, it is not clear from the literature whether the specimens were wild collected on the island (Ackerman, 2012). In Cuba it is reported for almost the whole island. However, we have seen differences in the morphology, color and fragrance of their flowers, so it would not be wrong to think that, under the epithet "phoenicea", several taxa are hiding. It would be premature and very risky to begin describing species considering the differences shown in this group. Only molecular studies could give really conclusive and accurate results.

Encyclia plicata (Lindl.) Schltr., Orchideen Beschreib. Kult. Zücht. 211. 1914.

BASIONYM: Epidendrum plicatum Lindl., Edward's Bot. Reg. 33: t. 35. 1847.

Type: Cuba: Loddiges s.n. (holotype K-L).

SPeCimEN STUdied: HOS!: flowers in spirit: Mújica 031.

Encyclia pyriformis (Lindl.) Schltr., Orchideen Beschreib. Kult. Zücht. 211. 1914. *

BASIONYM: Epidendrum pyriforme Lindl., Edward's Bot. Reg. 33: t. 15. 1847.

Encyclia pyriformis (Lindl.) Schltr., Orchideen 208. 1927.

Type: Cuba: Loddiges s.n. (K-L).

Epidendrum brevifolium Jenn., Annuaire Conserv. Jard. Genéve 11: 103. 1917.

Encyclia brevifolia (Jenn.) Ackerman \& Mújica,

Smithsonian Contr. Bot. 98: 631. 2012.

Type: Cuba: Isle of Pines (Isla de la Juventud), near Los Indios, Jenn. 314 (holotype CM).

SPecimens StUdied: HUJB!: Urquiola 242; HOS!: Pérez et al 007; flowers in spirit: Mújica \& Bocourt 030 . 
Encyclia rosariensis Múj.Benítez, R.Pérez, \& Pupulin, Orchids (West Palm Beach) 75: 677. 2006. *

Type: Cuba: Prov. Pinar del Río, Mun. Candelaria, El Salón, Sierra del Rosario Biosphere Reserve, $R$. Pérez 122 (holotype HOS!, isotype, HOS!).

Other SPECIMENS STUdied: HOS! flowers in spirit: Pérez \& Bocourt 040.

Encyclia sabanensis Vale, Pérez-Obregón \& Faife, Syst. Bot. 39. 2014. *

Type: Cuba: Caibarién: Cayo Santa María, holotype: UCLV: Pérez-Obregón 10593.

In the north of Villa Clara province, specifically in Cayo Santa María, there is a population of this orchid, very closely related to E. felhingii. One plant collected by the authors in November 2009 flowered in cultivation in the Soroa Orchid Botanical Garden. Later on, other individuals collected at the same location also flowered in cultivation. Vegetatively, it is indistinguishable from E. bocourtii, E. plicata and E. phoenicea. The flowers are morphologically very similar to those of $E$. bocourtii, but they differ in the green colored sepals and petals and the white labellum grooved in purple. Sauleda (pers. comm. 2011) suspects that it is not E. felhingii, but a natural hybrid between this species and E. plicata, which is to be studied.

Encyclia triangulifera (Rchb.f.) Acuña, Bol. Estación Exp. Agron. Santiago de las Vegas 60: 80. 1939. *

BAsionym: Epidendrum trianguliferum Rchb.f., Flora 48: 277. 1865; Type: Cuba: C. Wright 3328 (holotype K).

SPecimen STUdied: HAJB!: Dietrich 57672.

Encyclia vinalensis Múj.Benítez \& Rivera, ined.*

Cuba: Pinar del Río province, Valle de Viñales, El Sitio, Rivera 251 (HOS!).

Other SPecimens StUdied: Same locality as the type, Rivera 181 (HOS!: flowers in spirit)

The only known populations of E. vinalensis inhabit a fragile ecosystem on the limestone slopes of the "mogotes" in the Viñales National Park. The shape of the flowers, reveals some affinities with E. tampensis var. albolabia (Whitner, 1996, Plate 37). Nevertheless, the morphology of the plants and the flowering season (February and March) are quite distinct. The authors cannot find any close relatives of this taxon in Cuban flora. This species is now in description.
OTHER NOTES ON THIS GENUS:

\section{Encyclia $s p .1$}

Very closely related to E. oxypetala, with which, apparently, it was confused. The colleagues of Soroa Orchid Botanical Garden are working on its description. This species, reported only for Ciénaga de Zapata, Matanzas province, is distinguished from E. oxypetala by the smaller size of its flowers, darker coloration of its sepals and petals and its strong and unmistakable scent of "cockroach". It flowers in July-August.

\section{Encyclia sp. 2}

Present in Cabo San Antonio, Guanahacabibes peninsula, vegetatively very similar to other species of the genus and very closely related to E. fucata, from which it differs in the larger flowers and unguiculate, completely white-colored labellum. As E. fucata, it shows great variation in the floral morphology and color of the flowers. Both are in an area cloistered between the two coasts of the peninsula (not more than eight kilometers wide). We think, given the size of E. fucata, that it is not very likely for it to have been able to cross with other species such as E. bocourtii or E. plicata, so we think we have a new species just as suggested by P. Esperón and J. M. Díaz (pers. comm. 2012).

If the last three taxa were indeed accepted as good species, the number of Encyclia species in Cuba would reach 28 taxa. Nevertheless, the taxonomy of this genus in the island is still debatable, and the segregation of new taxa with limited information on the natural variation beteween and among populations, as well as the possible role of hybridization in the group, could result in gross mistakes.

\section{Epidendrum Linnaeus}

Epidendrum acunae Dressler, Amer. Orchid Soc. Bull. 28: 358. 1959.

Type: Cuba: Charles Wright 3333. (holotype: MO; isotypes: AMES, BM, G, K, MA, P x2, S, W x3).

Spathiger roigii Acuña, Cat. Descr. Orq. Cub. 60: 93. 1939.

Type: Cuba: Prov. Pinar del Río, P. Wilson 9329 (holotype NY).

Specimens Studied: HUJB!: Luis et al. 4054/4059; HOS!: Mújica et al. 191; flowers in spirit: Bocourt et al. 079. 
Epidendrum amphistomum A.Rich., in R.de la Sagra, Hist. Fis. Cuba Bot. 11: 240. 1850.

Type: Cuba: Ile de Cuba, 1836, Ramón de la Sagra s.n. (holotype: P).

Epidendrum secundum subsp. briegeri H.Dietr., Beitr. Phytotax. 15: 123. 1992.

Type: Cuba: Santiago de Cuba, Pico Cristal, cult. in Hort. Bot. Jenenese, H. Dietrich s.n. (holotype HAJB).

Specimens STUDIED: HUJB!: Ferro et al. 4307; HOS!: Mújica \& Elaine 216; flowers in spirit: Pérez \& Bocourt 019.

Epidendrum anceps Jacq., Select. Stirp. Amer. Hist. 224, t. 138. 1763.

Type: Dominica, Miller s.n. (holotype illustration, Botanical Cabinet 19: t. 1867. 1832).

Epidendrum anceps var. virescens (Lodd.) Lindl., in Hemsl., Biol. Centr. Am., Botany 3: 255. 1883.

Amphyglottis anceps (Jacq.) Britton, in Britton \& Wilson, Bot. Porto Rico 5: 200. 1924.

Epidendrum anceps var. typicum Stehlé (= var. anceps), Fl. Descr. Ant. Franc. 1: 135. 1939.

Type: Martinique, Jacquin, the illustration, plate 138 (BM).

SPeCimens STUdied: HUJB!: Ferro et al. 4307; HOS!: Pérez \& Bocourt 001; flowers in spirit: Pérez \& Bocourt 021.

Epidendrum angustilobum Fawc. \& Rendle, J. Bot. 47: 124. 1909.

Type: Jamaica: Rose Hill, Harris 10485 (holotype, cited by Nir, UCWI).

Epidendrum nocturnum var. latifolium Lindl., Bot. Reg. 23: t. 1961. 1837.

Amphyglottis nocturna var. latifolia (Lindl.) Acuña, Bol. Estación Exp. Agron. Santiago de las Vegas 60: 100. 1938.

Epidendrum latifolium (Lind1.) Garay \& Sweet, J. Arnold Arbor. 53: 392. 1972.

Type: West Indies, introduced and cultivated by Chatsworth, Paxton s.n. (K-L).

Specimen StUdied: HAJB!: Bisse et al. 22865.

Epidendrum brachyrepens Hágsater, Icon. Orchid. 3: pl. 315. 1999.

Type: Costa Rica: San José; Desamparados, San Cristóbal Sur, J. García-Cruz et al. 926 (holotype INB).
Specimen STUdied: HAC!: Morton et al. 12312.

Reported by Ackerman (2014) from the Sierra Maestra, Granma and Santiago de Cuba provinces. Eric Hágsater cites other herbaria specimens, including some of the HAC and HAJB, for Pico Turquino and one for Pico La Bayamesa in Santiago de Cuba province.

Epidendrum diffusum Sw., Prodr. Veg. Ind. Occ. 121. 1788 .

Type: Jamaica: O. Swartz s.n. (W 26526).

Seraphyta diffusa (Sw.) Fischer \& C.A.Meyer ex Pfitzer, in Engler \& Prantl, Nat. Pflanzenfam. 2(6): 142. 1889.

Seraphyta multiflora Fischer \& C.A.Meyer, Bul. Sc. Acad. Petersb. 7: 24. 1840.

Type: Mexico: Veracruz, C. Schiede s.n. (holotype LE).

Epidendrum tenuiflorum Hort. ex Lindl., Fol. Orchid. Epidendrum 88. 1853.

Type: México: Oaxaca, Galeotti 5234 (holotype P).

Epidendrum paniculatum Sessé \& Mociño, Flora Mexicana 204. 1894.

Type: Mexico: Veracruz, Montium Cordavae, Sessé et al. 4302 (MA).

Specimen STUdied: HAJB!: Alvarez et al. 55025.

Epidendrum floridense Hágsater, Icon. Orchid. 2: pl. 133. 1993.

Type: United States of America: Florida: Collier Co., Fahkahatchee Swamp, J. Corder sub E. Hágsater 1086 (holotype AMO).

SPECIMENS STUdied: HAJB!: Bisse et al. 26406; HUJB!: Urquiola et al. 6817; HOS!: flowers in spirit: Bocourt \& Pérez 061.

Epidendrum hioramii (Acuña \& Roig) Acuña \& Alain, Mem. Soc. Cub. Hist. Nat. "Felipe Poey" 24: 2. 110. 1960. *

Basionym: Hormidium hiorami Acuña \& Roig, Mem. Soc. Cub. Hist. Nat. "Felipe Poey" 10: 51. 1936.

Lanium hiorami (Acuña \& Alain) H.Dietr., Wiss. Z. Friedrich-Schiller-Univ. Jena. MathNaturwiss. Reihe 29: 522. 1980.

Type: Cuba: Guantánamo, Baracoa, Finca "Iberia", en Nibujón, J. Natenson s.n. (holotype HAC; isotype in the herbarium of Dr. J. T. Roig, No.7156).

Specimen Studied: HAC!: Alain et al. 4541. 
Epidendrum jamaicense Lindl., Fol. Orchid. Epidendrum 82, no.256. 1853.

Type: Jamaica: Dunrobin Castle, Purdie s.n. (holotype K-L).

SPecimen Studied: HAJB!: Bisse et al. 22685.

Epidendrum lacerum Lindl., Edward's Bot. Reg. 24 (Misc.): 18. 1838. *

Amphyglottis lacera (Lindl.) Britton, Ann. New York Acad. Sci. 5: 201. 1924.

Type: Cuba. Havannah, collected by Capt. Sutton, flowered at Charles Lemon, Cerclew, England, (holotype K-L).

Specimens studied: We did not find any specimens in the revised Cuban herbaria.

Introduced in England from "Havannah" (apparently Havana), Cuba, in the spring of 1835 by Captain Sutton. It flowered later at Charles Lemon's collection in November-December 1836. The species is either very rare or extinct.. In Cuba, E. lacerum is vegetative similar to $E$. wrightii Lindl., from which it differs by the pink flowers with the lateral lobes longer than apical (Hágsater, pers. comm. 2012).

Epidendrum miserrimum Rchb.f., Bonplandia 3: 220. 1855.

Type: Guadeloupe: Monte Fulsbunes, Duchassaing s.n. (holotype W 50153).

Jacquiniella miserrima (Rchb.f.) Stehlé, Bull. Soc. Bot. France 84: 425. 1937.

Microepidendrum miserrimum (Rchb.f.) Brieger, Rudolf Schltr.'s Orchideen, $3^{\text {rd }}$ ed., 556. 1974.

Specimens studied: It is known from a single collection of Wright (s.n.) from La Guinea, in eastern Cuba. We did not find any specimens in the reviewed Cuban herbaria.

Epidendrum neoporpax Ames, Bot. Mus. Leafl. 2(9):

112. 1934.

Type: Cuba: C. Wright 3343 (holotype W Isotypes: AMES G ( x2)! K).

BASIONYM: Epidendrum porpax Rchb.f., Flora 48: 278. 1865, non Rchb.f. 1855.

Epidendrum vestitum Ames, Schedul. Orchid. 4: 51. 1923, non Sw., 1788.

Epidendrum porpax var. domingensis Cogn., in

I.Urban, Symb. Antill. 7: 181. 1909.

Specimens Studied: HAC!: Morton 20179; HOS!: Collector unknown 120; flowers in spirit: collector unknown 037.
Epidendrum nocturnum Jacq., Enum. Syst. P1. 29. 1760.

Auliza nocturna (Jacq.) Small, Fl. Miami: 56. 1913.

Amphyglottis nocturna (Jacq.) Britton, Bot. Porto

Rico \& Virgin Islands 1: 200. 1924.

Phaedrosanthus nocturnus (Jacq.) Kuntze in T.

Post \& Kuntze, Lex. gen. phan. 429. 1904.

Type: Martinique, Jacquin s.n. (lectotype, Jacquin's illustration reproduced in Selectarum Stirpium Americanarum Historia 225, t. 139. 1763).

Epidendrum nocturnum var. angustifolium Stehlé, Fl. Descr. Antill. Fr. 1: 143. 1939.

Epidendrum carolinianum Lam., Encycl. 1: 182. 1783.

Lectotype: Gatesby, Nat. Hist. Carol. 2: t. 68 (1743). Epidendrum discolor A. Rich. \& Gal., Ann. Sci. Nat. ser. 3. 3: 22. 1845.

Type: Mexico: Galeotti s.n. t. 18.

SPeCiMENS StUdied: HUJB!: Urquiola et al. 508; HOS!: Mújica \& Elaine 198; flowers in spirit: Trabanco 060.

Epidendrum orientale Hágsater \& M.A.Díaz, Icon. Orchid. 2: pl. 167. 1993.

Type: Cuba: Guantánamo, Laguna del Galano, Sierra del Frijol, La Alegria, Toa, Hno. Alain 3854 (holotype HAC!).

SPECIMENS STUdIED: HPPR!: Ferro et al. s.n.; HOS!: Pérez et al. 085; flowers in spirit: Pérez \& Bocourt 013.

Epidendrum polygonatum Lindl., Ann. Mag. Nat. Hist. series 3, 1: 332. 1858.

Amphyglottis polygonata (Lindl.) Acuña, Cat. Descr. Orq. Cub. 60: 101. 1939.

Physinga polygonata (Lindl.) Dod. Moscosoa 3: 101-102. 1984.

Physinga polygonata (Lindl.) H. Dietrich. Wiss.

Zeitschr. Friedrich-Schiller-Univ. Jena, Mat.

Naturwiss. (Beitr. Phytotax.) 29 (4): 524, 1980. Type: Cuba: Popre villam, Monteverde dictam, $C$. Wright $643 \mathrm{P}$ !.

Epidendrum polygonatum Lindl. var. latifolium Cogn., Symb. Antill. 6: 514. 1910.

Type: Cuba: Pinal de Sta. Anna, 800 m, Eggers 5053, (holotype: BR).

SPECIMENS STUdied: HAJB!: Bisse et al. 27680; HPPR!: Urquiola et al. 6318-A. 
Epidendrum portoricense Hágsater \& Ackerman, Icon. Orchid. 3: 2, pl. 376. 1999.

Type: Puerto Rico: Mun. Río Grande, Luquillo Mts., El Verde, Ackerman 2613 (holotype UPRRP). SPECIMENS STUdied: We did not find any specimens in the revised Cuban herbaria. Reported by Ackerman (2014) for Macizo Sagua-Baracoa, Guantánamo province.

Epidendrum radicans Pav. ex Lindl., Gen. Sp. Orchid. Pl. 104. 1831.

Type: Mexico, Sessé \& Mociño, (holotype BM). Epidendrum rhizophorum Batem. ex Lindl., Edward's Bot. Reg. 24 (Misc.): 8. 1838.

Type: Guatemala, Skinner s.n. (holotype ?).

Epidendrum pratense Rchb.f., Beitr. Orch. Centr. Amer. 84. 1866.

Type: Guatemala, Wendland 271 (holotype W). Epidendrum radicans var. chiriquense Schltr., Repert. Spec. Nov. Regni Veg. 17: 39. 1922. Type: Panama, Powell 61 (holotype B, destroyed). Specimens Studied: We did not find any specimens in the reviewed Cuban herbaria.

This species was introduced and it is in naturalization process in Cuba. It has been collected by specialists of the Soroa Orchid Botanical Garden in several occasions in the proximities of La Gran Piedra, Santiago de Cuba province. We suppose that around there it may have escaped from cultivation.

Epidendrum ramosum Jacq., Enum. Syst. P1. 29. 1760.

Spathiger ramosus (Jacq.) Britton \& Wilson, Sci. Surv. Porto Rico and Virg. Isl. 5: 202. 1924.

Isochilus ramosum (Jacq.) Spreng., Syst. Veg. ed. 16, 3: 734. 1836, non H.Focke, 1851.

Type: Martinique, Jacquin S.n. (holotype LINN).

Epidendrum rigidum Lodd., Bot. Cab. 16: t. 1600. 1829.

Type: Dominica, Miller; non Jacq. 1760.

SPECIMENS STUdiEd: HAJB!: Alvarez et al. 64786; HOS!: Félix \& Sandy 104; flowers in spirit: collector unknown 165.

Epidendrum repens Cogn., Repert. Spec. Nov. Regni Veg. 7: 122. 1909.

Type: Jamaica, H. Eggers 3679 (BR).

Epidendrum bourgeaui Schltr., Beih. Bot. Centralbl. 36: 401. 1918,
Epidendrum solisequum Kraenzl, Ark. Bot. 16: 17, 1920.

Type: Mexico, E. Borgeau 3104, (holotype B, destroyed; isotypes $\mathrm{BR}, \mathrm{GH}, \mathrm{LE}, \mathrm{P}, \mathrm{P}, \mathrm{US}$ ).

SPECIMEN STUdied: HAJB!: Bisse et al. 40518.

Epidendrum rigidum Jacq., Enum. Syst. P1. 29. 1760.

Spathiger rigidus (Jacq.) Small, Fl. Miami 55. 1913.

Type: Martinique, Jacquin s.n. (holotype BM).

SPECIMENS STUdied: HUJB!: Bisse et al. s.n.; HOS!:

Mújica \& Elaine 209.

Epidendrum rivulare Lindl., Ann. Mag. Nat. Hist., ser. 3, 1:330. 1858.

Type: Cuba Orientali, Prope villam, Monte Verde,

C. Wright 644 (holotype K; istoypes AMES, BR, $\mathrm{G}, \mathrm{P}, \mathrm{W})$.

Specimen StUdied: HAJB!: Bisse et al. 19683.

Epidendrum scalpelligerum Rchb.f., Flora 48: 278. 1865.

Pleuranthium scalpelligerum (Rchb.f.) Cogn., in I.Urban, Symb. Antill. 4: 470. 1920.

Type: Cuba: Without locality, C. Wright 3339 (holotype K-illustration; isotypes AMES, G, P,W). SPeCimens studied: We did not find any specimens in the reviewed Cuban herbaria. Reported by Ackerman (2014) from Macizo Sagua-Baracoa, Guantánamo province and by Hágsater (pers. comm. 2012) from La Perla, Santiago de Cuba province.

Epidendrum serrulatum Sw., Prodr. Veg. Ind. Occ. 121. 1788.

Cymbidium serrulatum (Sw.) Sw., Nova Acta Regiae Soc. Sci. Upsal. 6: 72. 1799.

Encyclia serrulata (Sw.) H.Dietr., Rev. Jard. Bot. Nac. La Habana 5(1): 49. 1984.

Prosthechea serrulata (Sw.) W.E.Higgins, Phytologia 82: 380. 1997.

Type: Jamaica: Summit Mont. Coral, Swartz s.n. (holotype BM).

Specimen Studied: HAJB!: Bisse et al. 19065.

Epidendrum strobiliferum Rchb.f., Nederl. Kruidk. Arch. 4: 333. 1858.

Spathiger strobiliferus (Rchb.f.) Small, Man. SE. Fl. 390. 1933.

Type: Surinam, Splitgerber 426 (holotype W).

Isochilus ramosus H.Focke, Tijdschr. Natuurk.

Wetensch. Kunsten 4: 69. 1851. 
Type: Surinam, H.Focke s.n. (holotype ?).

SPecimen Studied: HAJB!: Bisse et al. 45376.

Epidendrum umbelliferum J.F.Gmel, Syst. Nat., ed. 13, 2(1): 65. 1791.

Type: Jacquin, Stirp. Pl. Amer. pl. 136.

Epidendrum umbellatum Sw., Nova Spec. Pl. Prodr. 121. 1788, nom. illeg.

Caularthron umbellatum (Sw.) Rafin., Fl. Tellur. 2: 41.1837.

Epidendrum caribiorum Ackerman \& Acev.Rodr., Smithsonian Contr. Bot. 98: 633. 2012

(Based on Epidendrum umbellatum Sw., non G. Forster).

Type: Jamaica, without locality, Swartz s.n. (holotype BM).

Specimens STUdied: HAJB!: Bisse et al. 48056; HOS!: flowers in spirit: Pérez \& Bocourt 073.

Epidendrum wrightii Lindl., Ann. Mag. Nat. Hist. series 3, 1: 331. 1858.

Type: Cuba: province unknown, Santa $\mathrm{La}$ Madelina, C. Wright s.n. (holotype K-L).

Specimen StUdied: HAJB!: Dietrich et al. 66743.

\section{Eulophia R. Brown}

Eulophia alta (L.) Fawc. \& Rendle, Fl. Jamaica 1: 112, plate 22. 1910.

Basionym: Limodorum altum L., Syst. Nat., ed. 12, 594. 1767.

Platypus altus (L.) Small, Flora of the S.E. of U.S., $2^{\text {nd }}$ ed., 329. 1913.

Type: Martinique, Plumier s.n. (original illustration at $\mathrm{P})$.

Cyrtopodium woodfordii Sims, Bot. Mag. 43: t. 1814. 1816.

Cyrtopera woodfordii (Sims) Lindl., 189. 1833.

Eulophia woodfordii (Sims) Rolfe, Fl. of Trop. Africa 7: 68. 1897.

Type: Brazil, Sao Paulo, J. Sims s.n., (K).

Dendrobium longifolium Kunth, in Humboldt, Bonpland, \& Kunth, Prodr. Veg. Ind. Occ.1: 360. 1815.

Cyrtopera longifolia (Kunth) Rchb.f., in W.G.Walpers, Ann. Bot. Syst. 6: 669. 1863.

Eulophia longifolia (Kunth) Schltr., Orchideen 347. 1914.

Type: Colombia, Almaguer, Humboldt \& Bonpland 1067 (Holotype P).
Specimens StUdied: HAJB!: Bisse 55150; HOS!: flowers in spirit: Eduard 059.

\section{Eurystyles Wawra}

Eurystyles domingensis Dod, Moscosoa 1: 43. 1977. Type: Dominican Republic: Prov. San Cristóbal, Villa Altagracia, confluencia Río Haina y Río Duey, D. D. Dod 475 (holotype JBSD).

Specimens studied: We did not find any specimens in the reviewed Cuban herbaria.

According to Ackerman (2014) the species that is in Cuba is really $E$. domingensis and not $E$. ananassocomus (Rchb. f.) Schltr. The specimens revised by him, from Monte Verde and La Prenda demonstrated that with certainty. However, in the HAJB there is a specimen from another locality in Sierra del Purial (Bisse et al. 47265) identified as E. ananassocomus that deserves to be studied.

FuERTESIELLA Schlechter

Fuertesiella pterichoides Schltr., in I.Urban Symb. Antill. 7: 493. 1919.

Type: Dominican Republic: Prov. La Vega, between Rosilla and Picos del Yaque, Fuertes 1718 (holotype B, destroyed; lectotype NY).

SPECIMEN STUDIED: HAC!: Acuña 10013.

\section{Galeandra Lindley}

Galeandra bicarinata G.A.Romero \& P.M.Brown, North Amer. Nat. Orchid Jour. 6: 78. 2000.

Type: United States of America: Florida, MiamiDade County, Castellow's Hammock, $R$. Woodbury \& K. Kramer s.n. (holotype FTG).

Specimens studied: HAC!: Alain 6530; HOS!: Mújica et al. 192.

\section{Govenia Lindley ex Loddiges}

Govenia utriculata (Sw.) Lindl., Edward's Bot. Reg. 25 (Misc.): 47. 1839.

BASIONym: Limodorum utriculatum Sw., Prodr. Veg. Ind. Occ. 119. 1788.

Cymbidium utriculatum (Sw.) Sw., Nova Acta

Regiae Soc. Sci. Upsal. 6: 75. 1799.

Type: Jamaica: O. Swartz s.n. (BM ).

Specimens studied: HAJB!: Meyer et al. 36264; HUCLV!: Noa et al. 2779. 


\section{|Habenaria Willdenow}

Habenaria alata Hook., Exot. Fl. 3: t. 169. 1825. Habenella alata (Hook.) Szlach. \& Kras-Lapinski, Richardiana 6: 34. 2006.

Type: St. Vincent, Guilding s.n. (holotype K).

Platantheroides alata (Hook.) Szlachetko, Richardiana 4: 104. 2004.

Habenaria bidentata Steud., Nomencl. Bot. ed. 2, 1: 716.1840.

Type: Cuba, E. Pöppig s.n. (MO).

Habenaria stricta A.Rich. \& Gal. Ann. Sci. Nat., Bot. ser. 3, 3: 23. 1845.

Type: Mexico: Veracruz, Mirador, Linden s.n. (P). Habenaria triptera Rchb.f., Linnaea 22: 814. 1849. Syntypes: Mexico: Mesochiza [Veracruz, Mirador, Mesa Chica], Schiede s.n. (BM); Mexico, [Veracruz], Zacuapan, Leibold s.n. (W).

Habenaria platantheroides Schltr., Beih. Bot. Centralbl., Abt. 2, 36(2): 372. 1918.

Type: Costa Rica: La Palma, 1913, C. Wercklé s.n. (holotype B, destroyed).

Specimens studied: HAC!: Acuña 16443; HUJB!: Urquiola et al. 5795.

Habenaria bicornis Lindl., Gen. Sp. Orchid. Pl. 309. 1835.*

Type: Cuba, Pöppig s.n. (holotype K).

Specimens Studied: We did not find any specimens in the reviewed Cuban herbaria. Reported by Ackerman (2014) from Isla de la Juventud, Pinar del Río (Llanura Aluvial del Sur), Matanzas (Ciénaga de Zapata), Santiago de Cuba, and Villa Clara; Granma (Llanura del Cauto-Guacanayabo), and Artemisa (Sierra del Rosario).

Habenaria brittonae Ames, Torreya 12: 11. 1912.* Type: Cuba: Prov. Pinar del Río, vicinity of Venales (Viñales), N. L. \& E. G. Britton 7540 (holotype AMES).

Specimens STUdied: HAC!: Acuña et al. 18825.

Habenaria distans Griseb., Cat. Pl. Cub. 270. 1866.

Type: Cuba: Monteverde, Wright 1481 (holotype GOET).

Habenaria jamaicensis Fawc. \& Rendle, J. Bot. 47: 126. 1909.

Habenaria distans var. jamaicensis (Fawc. \& Rendle) Cogn., in I.Urban, Symb. Antill. 6: 300. 1909.
Type: Jamaica, Cinchona, Harris 10449 (holotype $\mathrm{BM})$.

Specimens studied: HAJB!: Arias et al. 60086; Borhidi 32861.

Habenaria eustachya Rchb.f., Ber. Deutsch. Bot. Ges. 3: 274.1885 .

Habenella eustachya (Rchb.f.) Szlach. \& KrasLapinski, Richardiana 6: 35. 2006.

Type: Puerto Rico: near Maricao, Sintenis $511 b$ (holotype W).

Habenaria sanbornii Ames, Proc. Biol. Soc. Wash. 16: 117. 1903.

Type: Cuba: Prov. Artemisa, Cayajabos, Ames \& Leavitt 519 (holotype AMES).

Habenaria troyana Fawc. \& Rendle, J. Bot. 47: 264. 1909.

Type: Jamaica, near Troy, Harris 10432 (holotype $\mathrm{BM})$.

Habenaria socialis Fawc. \& Rendle, J. Bot. 47: 263. 1909.

Type: Jamaica, near Mandeville, Manchester, Purdie s.n. (holotype K).

SPecimens studied: HAC!: Alain 6895; HOS!: Sandy 094.

Habenaria floribunda Lindl., Gen. Sp. Orch. Pl. 316. 1835. Habenella floribunda (Lindl.) Szlach. \& KrasLapinski, Richardiana 6: 36. 2006.

Type: Peru: Haenke s.n. (holotype M).

Habenaria autumnalis Poepp. \& Endl., Nov. Gen. Spec. Plant. 1: 44, t. 75. 1836.

Type: Peru: inter praedia Cassapi et Paampayaco (holotype W).

Habenaria odontopetala Rchb.f., Linnaea 18: 407. 1844

Habenella odontopetala (Rchb.f.) Small, Man. SE. Fl. 373. 1933.

Habenaria strictissima var. odontopetala (Rchb.f.) L.O.Williams, Bot. Mus. Leafl. 7: 184. 1939.

Lectotype (designated by Batista et al. 2011): Mexico, F.E. Leibold s.n. (W-R).

Specimens Studied: HAC!: López Figueiras 19845; HOS!: Bocourt 234; flowers in spirit: Bocourt et al. 081.

Habenaria monorrhiza (Sw.) Rchb.f., Ber. Deutsch. Bot. Ges. 3: 274. 1885.

Basionym: Orchis monorrhiza Sw., Prodr. Veg. Ind. Occ. 118. 1788. 
Type: Jamaica, Swartz s.n. (holotype BM).

Habenaria brachyceras Spreng., Syst. Veg. ed. 16, 3: 692.1826.

Orchis setacea Jacq., Enum. Syst. P1. 29. 1760.

Type: Martinique: Jacquin s.n. (BM?).

Specimen studied: HAJB!: Arias et al. 62870.

Habenaria quinqueseta (Michx.) Sw., Adnotat. Bot. 46. 1829.

Basionym: Orchis quinqueseta Michx., F. Bor. Amer. 2: 155. 1803.

Mesicera quinqueseta (Michx.) Raf., Neogenyton 4. 1825.

Type: United States of America: Carolina, Michaux s.n. (holotype P).

Habenaria michauxii Nutt., Gen. N. Amer. P1. 2: 189. 1818.

Mesicera michauxii (Nutt.) Raf., Fl. Tellur. 2: 39. 1836.

Platanthera michauxii (Nutt.) Wood, Class-book 685. 1861.

Orchis michauxii (Nutt.) Wood, Amer. Bot. Fl. 328. 1871.

Specimens STUdied: HAJB!: Bisse \& Díaz 46697; HAC!: Acuña 9774; HOS!: Pérez et al. 037.

Habenaria repens Nutt., Gen. N. Amer. P1. 2: 190. 1818.

Orchis repens (Nutt.) Raf., Neogenyton 4. 1825.

Platanthera repens (Nutt.) Wood, Class-book 685. 1861.

Type: United States of America: Georgia, Savannah, Nuttall s.n. (PH).

Habenaria tricuspis A.Rich. in R.de la Sagra, Hist. Fis. Cuba Bot. 11: 249. 1850.

Type: Cuba: Ramón de la Sagra s.n. (holotype P). Habenaria radicans Griseb., Cat. Pl. Cub. 271. 1866.

Habenaria nuttallii Small, Fl. S.E. U.S. 315. 1903. Type: United States of America: Florida, Sumter Co., A. H. Curtiss 2772 (holotype NY).

Habenaria palustris Acuña, Bol. Estación Exp. Agron. Santiago de las Vegas 60: 15. 1939.

Type: Cuba: Province Villa Clara??, Near Santo Tomás, Ciénaga de Zapata, Roig \& Cremata s.n., herb. J. T. Roig No. 2211 (holotype HAC!).

Specimen STUdied: HAJB!: Arias et al. 51022.
HAPALORCHIS Schlechter

Hapalorchis lineatus (Lindl.) Schltr., Beih. Bot. Centralbl., Abt. 2, 37(2): 363. 1920.

Basionym: Spiranthes lineata Lindl., Gen. Sp. Orchid. P1. 471. 1840.

Gyrostachys lineata (Lindl.) Kuntze, Revis. Gen. P1. 2: 664. 1891.

Cyclopogon lineatus (Lindl.) Pabst, Bradea 1(47): 466. 1974.

Type: Brazil: Sao Paulo, Descourtilz 35 (holotype K-L).

Sauroglossum tenue Lindl., Ann. Mag. Nat. Hist., Ser. 3, 1: 334. 1858.

Spiranthes fawcetii Cogn., Feddes Repert. Spec. Nov. Regni Veg. 7: 123. 1909.

Type: Cuba, Monteverde, C. Wright 622 (holotype K-L).

Spiranthes amabilis Ames, Schedul. Orchid. 2: 8. 1923.

Cyclopogon amabilis (Ames) Acuña, Bol. Estación Exp. Agron. Santiago de las Vegas 60: 35. 1939.

Type: Guatemala, Alta Verapaz, Cobán, H. v. Tuerckheim II 1787 (holotype US).

Specimens StUdied: HAJB!: Bisse et al. 52471; HOS!: Pérez et al. 093.

\section{Homalopetalum Rolfe}

Homalopetalum leochilus (Rchb.f.) Soto Arenas, Neodiversity 2: 8. 2007.

Basionym: Epidendrum leochilus Rchb.f., Flora 48: 277. 1865.

Hormidium leochilus (Rchb.f.) B.D.Jacks., Index Kew. 1: 1174. 1893.

Pinelia leochilus (Rchb.f.) Garay \& H.R.Sweet, J. Arnold Arbor. 53: 394. 1972.

Type: Cuba: Prov. Guantánamo, near Monte Verde, C. Wright 3346 (holotype K).

Specimens studied: HAJB!: Bisse et al. 52381.

Homalopetalum vomeriforme (Sw.) Fawc. \& Rendle, Fl. Jamaica 1: 106. 1910.

Basionym: Epidendrum vomeriforme Sw., Prodr. Veg. Ind. Occ. 124. 1788.

Brassavola vomeriformis (Sw.) Rchb.f. ex Griseb., Fl. Brit. W. I. 621. 1864.

Type: Jamaica: Swartz s.n. (BM). 
Homalopetalum jamaicense Rolfe, Hooker's Icon. Pl. t. 2461. 1896.

Type: Jamaica: Blue Mountains above Abbey Green coffee plantation, W. Harris 6107 (K).

SPECIMENS STUDIED: We did not find any specimens in the reviewed Cuban herbaria. Reported for Sierra Maestra, Santiago de Cuba province (Ackerman, 2014).

\section{IoNOPSIS Kunth}

Ionopsis satyrioides (Sw.) Rchb.f., in W.G.Walpers, Ann. Bot. Syst. 6: 683. 1863.

Basionym: Epidendrum satyrioides Sw., Prodr. Veg. Ind. Occ. 123. 1788.

Type: Jamaica, Swartz s.n. (BM).

SPECIMENS STUdiEd: HAJB!: Alvarez et al. 57070; HOS!: Pérez et al. 083.

Ionopsis utricularioides (Sw.) Lindl., Coll. Bot. t. 39A. 1826.

Basionym: Epidendrum utricularioides Sw., Prodr. Veg. Ind. Occ. 122. 1788.

Type: Jamaica, Swartz s.n. (BM).

Ionopsis utricularioides forma latifolia Urb., in I.Urban, Symb. Antill. 4: 180. 1903.

Type: Puerto Rico, Adjuntas, P. Sintenis 4407 (isotype $\mathrm{BM}$ ).

SPecimens STUdied: HAJB!: Bisse et al. 46320; HOS!: Mújica \& Elaine 196; flowers in spirit: Martínez 005.

\section{IsochILUs R. Brown}

Isochilus linearis (Jacq.) R.Br., in W.T.Aiton, Hort. Kew. 5: 209. 1813.

BAsionym: Epidendrum lineare Jacq., Enum. Syst. P1. 29. 1760.

Cymbidium lineare (Jacq.) Sw., Nova Acta Regiae Soc. Sci. Upsal. 6: 72. 1799.

Type: Martinique: Jacquin s.n. (original illustration at $\mathrm{P}$ ).

Specimens STUdied: HUJB!: Urquiola et al. 5192; HOS!: Mújica \& Bocourt 177.

\section{JACQUinIELLa Schlechter}

Jacquiniella globosa (Jacq.) Schltr., Repert. Spec. Nov. Regni Veg. 7: 124. 1920.

BAsIonym: Epidendrum globosum Jacq., Enum. Syst. P1. 29. 1760.
Isochilus globosus (Jacq.) Lindl., Gen. Sp. Orchid. P1. 112. 1831.

Type: Martinique, Jacquin s.n. (holotype BM).

SPECIMENS STUDIED: HUJB!: Urquiola et al. 6358; HOS!: flowers in spirit: Pérez 050.

Jacquiniella teretifolia (Sw.) Britton \& Wilson, Sci. Surv. Porto Rico \& Virgin Islands 6: 340. 1926.

Basionym: Epidendrum teretifolium Sw., Prodr. Veg. Ind. Occ. 121. 1788.

Cymbidium teretifolium (Sw.) Sw., Nova Acta Regiae Soc. Sci. Upsal. 6: 72. 1799.

Isochilus teretifolius (Sw.) Lindl., Gen. Sp. Orchid. P1. 112. 1831.

Epidendrum teretifolium var. powellianum Schltr., Repert. Spec. Nov. Regni Veg. 17: 41. 1922.

Briegeria teretifolia (Sw.) Senghas, Orchidee 31: 30. 1980.

Type: Jamaica: Swartz s.n. (BM).

SPeCIMENS STUdied: HAJB!: Bisse et al. 49147; HUCLV: Noa \& Castañeda 2856; HOS!: Pérez et al. 092.

\section{KREODAnThus Garay}

Kreodanthus corniculata (Rchb.f.) Garay, Bradea 2: 198. 1977.*

Basionym: Physurus corniculatus Rchb.f., Flora 48: 274. 1865.

Erythrodes corniculatus (Rchb.f.) Carabia, Mem.

Soc. Cub. Hist. Nat. 17: 146. 1943.

Goodyera corniculata (Rchb.f.) Ackerman, in

Acevedo-Rodríguez \& Strong, Catalogue of

Seed Plants of the West Indies 638. 2012.

Type: Cuba: Prov. Guantánamo, Monteverde, Potosí, Mt. Toro, C. Wright 3295 (holotype K). SPeCimen STUdied: HAC!: Roig et al. 13897.

Ackerman (2014) made a new combination and placed this species in Goodyera. However, Gerardo Salazar (pers. comm. 2014) considers that the South American and Caribbean species of Kreodanthus are part of another group and it does not justify their transference to Goodyera.

\section{LAELIA Lindley}

Laelia lyonsii (Lindl.) L.O.Williams, Darwiniana 5: 76. 1941.

BASIOnYM: Schomburgkia lyonsii Lindl., Gard. 
Chron. 1: 615. 1853.

Bletia lyonsii (Lindl.) Rchb.f., in W.G.Walpers, Ann. Bot. Syst. 6: 418. 1861.

Type: country of origin unknown, cultivated by J.

C. Lyons of Ireland who had obtained it from Mr. Clowes (holotype K).

Schomburgkia carinata Griseb., Fl. Brit. W. I. 621. 1864.

Type: Jamaica: St. Ann, Purdie s.n. (holotype K-L).

Specimen studied: HAC!: Casas de Almeida 14621.

\section{LANKeSTERELla Ames}

Lankesterella alainii Nir, Orchid. Antill. 170. 2000. Type: Dominican Republic: Prov. La Vega, Loma del Campanario, La Cuolata, Constanza, Liogier 17114 (holotype NY).

SPecimen STUdied: HAC!: Acuña 10010.

\section{LeOCHILus Knowles \& Westcott}

Leochilus labiatus (Sw.) Kuntze, Revis. Gen. P1. 2: 656. 1891.

Basionym: Epidendrum labiatum Sw., Prodr. Veg. Ind. Occ. 124. 1788.

Liparis labiata (Sw.) Spreng., Syst. Veg. 3: 71. 1826.

Oncidium labiatum (Sw.) Rchb.f., in W.G.Walpers,

Edward's Bot. Reg. 28 (Misc.): 22. 1842.

Type: Haiti: O. Swartz s.n. (lectotype S).

Rodriguezia cochlearis Lindl., Ann. Nat. Hist. 5: 116. 1840

Leochilus cochlearis (Lindl.) Lindl., Edward's

Bot. Reg. 28 (Misc.): 23. 1842.

Type: Dominica: Henslow s.n. (holotype K-L).

Oncidium salvum Rchb.f., Flora 48: 278. 1865.

Leochilus salvus (Rchb.f.) Griseb., Cat. Pl. Cub. 267. 1866.

Type: Cuba: 1860-1864, Wright 3311 (holotype $\mathrm{K})$.

SPeCIMENS STUdied: HAJB!: Bisse et al. 49093; HUCLV: Hernández 5764; HOS!: Mújica et al. 186; flowers in spirit: Martinez 004.

\section{LEPANTHES Swartz}

Lepanthes acunae Hespenh., Brittonia 25: 263. 1973.* Type: Cuba: Prov. Santiago de Cuba: Sierra
Maestra, between Pico de Turquino and La Bayamesa, Morton \& Acuña 3675 (holotype US).

SPeCimENS STUdied: We did not find any specimens in the reviewed Cuban herbaria.

Lepanthes aubryi Luer \& H.P.Jesup, Selbyana 23: 4. 2002.*

Type: Cuba: Santiago de Cuba: Sierra Maestra, Pico Turquino, collected by Yves Aubry, Connecticut, Jesup 8 (holotype MO).

SPECIMEN STUDIED: HAJB: Aubry s.n.

Lepanthes blepharantha Schltr., in I.Urban, Symb. Antill. 9: 61. 1923.*

Type: Cuba: Santiago de Cuba: Sierra Maestra, north slopes of Pico Turquino, E. L. Ekman 5491 (holotype S).

SPECIMEN STUdied: HAC!: Acuña $s / n$.

Lepanthes blepharophylla (Griseb.) Hespenh., Brittonia 25: 260. 1973.*

Basionym: Pleurothallis blepharophylla Griseb., Cat. P1. Cub. 260. 1866.

Lepanthopsis blepharophylla (Griseb.) Garay, J. Arnold Arbor. 50: 464. 1969.

Type: Cuba: Guantánamo: Sierra de Toa (?), near Monte Verde, 1859, C. Wright 1508 (holotype GOET).

Lepanthes ciliata Lindl. ex Griseb., Cat. Pl. Cub., 260. 1866, nomen nudum.

SPeCiMEN STUdied: HAC!: Wright 1508.

Lepanthes caluffii González \& Luer, Orchids (West Palm Beach). 78: 11. 674-675. 2009.*

Type: Cuba. Prov. Santiago de Cuba, between La Mercedita and La Isabelita, La Gran Piedra, Sierra Maestra, (holotype, HOS!: R. Pérez No.172).

Lepanthes chrysostigma Lindl., Ann. Mag. Nat. Hist., series 3, 1: 329. 1858.*

Type: Cuba: Oriente: Sierra de Toa (?), near Monte Verde, 1859, C. Wright s.n. (holotype K).

Lepanthes mandibularis Rchb.f., Flora 48: 275. 1865.

Type: Cuba: Oriente: C. Wright 1342 (holotype K). SPECIMENS STUDIED: We did not find any specimens in the reviewed Cuban herbaria.

Lepanthes comadresina Luer, Lindleyana 13: 138. 1998.*

Type: Cuba: Holguín: Moa, camino a La Melba, Arroyo Las Comadres, Luer et al. 18650 (holotype HAJB!). 
Lepanthes cubensis Hespenh., Brittonia 25: 269. 1973.*

Type: Cuba: Prov. Guantánamo: Sierra de Toa (?), near Monte Verde, 1859-1860, C. Wright 1512 (holotype $\mathrm{PH}$ ).

Specimen Studied: HAC!: Wright 1512.

Lepanthes cyrillicola Luer \& Llamacho, Selbyana 22: 104. 2001.*

Type: Cuba: Prov. Santiago de Cuba: Sierra Maestra, Pico Turquino, J. Llamacho 0014 (holotype HAC!).

Lepanthes decoris Luer \& Llamacho, Selbyana 22: 106. 2001.*

Type: Cuba: Santiago de Cuba: Sierra Maestra, Pico de Turquino, paso de Mono, J. Llamacho 0010A (holotype HAC!).

Lepanthes diaziae Luer, Lindleyana 13: 138. 1998.*

Type: Cuba: Holguín: Mayarí, Sierra de Nipe, Cayo Las Mujeres, Luer et al. 18622 (holotype HAJB!).

Lepanthes dorsalis Lindl., Ann. Mag. Nat. Hist., series 3, 1: 329. 1858.*

Type: Cuba: Santiago de Cuba: Oriente, Sierra Maestra, summit of Nemanima, 1856-57, C. Wright 662 (holotype K).

SPecimens studied: We did not find any specimens in the reviewed Cuban herbaria.

Lepanthes dressleri Hespenh., Brittonia 25: 268. 1973.*

Type: Cuba: Prov. Cienfuegos: Pico Sombrero, northeast of El Naranjo, Sierra Trinidad, $R$. L . Dressler 1341 (holotype US).

Specimens studied: HOS!: Bocourt et al. 145; flowers in spirit: Bocourt et al.080.

Lepanthes ekmanii Schltr., in I.Urban, Symb. Antill. 9: 62. 1923.*

Type: Cuba: Santiago de Cuba: Sierra Maestra, Pico Turquino, E. L. Ekman 5409 (holotype S).

Lepanthes ovata Ames \& C.Schweinf., Schedul. Orchid. 8: 37. 1925.

Type: Cuba: Santiago de Cuba, Sierra Maestra, León 10709 (NY).

SPecimens studied: We did not find any specimens in the reviewed Cuban herbaria.

Lepanthes fractiflexa Ames \& C.Schweinf., Schedul. Orchid. 10: 42. 1930. *

Type: Cuba: Prov. Santiago de Cuba: Sierra
Maestra, Cueva del Aura, Loma Cardero, Pico Turquino, G. C. Rucker 30 (holotype NY).

SPecimen STUdied: HAC!: Roig \& Acuña $s / n$.

Lepanthes fulva Lindl., Ann. Mag. Nat. Hist., series 3, 1: 329. 1858.*

Type: Cuba: Prov. Guantánamo: Sierra de Toa (¿), near Monte Verde, C. Wright s.n. (holotype K).

Lepanthes lindmaniana Schltr., in I.Urban, Symb. Antill. 9: 63. 1923.

Type: Cuba: Prov. Santiago de Cuba: Sierra Maestra, Loma del Gato, E. L. Ekman 6965 (holotype S).

Lepanthes occidentalis Hespenh., Brittonia 25: 276. 1973.

Type: Cuba: Prov. Villa Clara, Manicaragua, Dressler 1294 (holotype US).

Specimens studied: HAC!: Alain 4586; HAC!: Alain \& Figueiras 4586.

Lepanthes grisebachiana Hespenh., Brittonia 25: 272. 1973.*

Type: Cuba: Prov. Guantánamo: Cuchillas del Toa, near Monte Verde, C. Wright 1510 (holotype PH).

SPeCimens STUdied: We did not find any specimens in the revised Cuban herbaria.

Lepanthes llamachoi Luer, Lindleyana 13: 141. 1998.* Type: Cuba: Holguín: camino a La Melba, Arroyo Las Comadres, Luer et al. 18651 (holotype HAJB!).

Lepanthes melanocaulon Schltr., in I.Urban, Symb. Antill. 9: 64. 1923.

Type: Cuba: Prov. Holguín: Sierra de Nipe, Río Piloto, E. L. Ekman 3361 (holotype S).

Specimens studied: HAC!: Alain 1514; Alain 6981. Lepanthes nana Luer \& H.P.Jesup, Selbyana 23: 17. 2002.*

Type: Cuba: Santiago de Cuba: Sierra Maestra, Pico de Santiago, P. Jesup s.n. (holotype MO).

SPecimens Studied: We did not find any specimens in the reviewed Cuban herbaria.

Lepanthes obliquiloba Hespenh., Brittonia 25: 273. 1973. *

Type: Cuba: Prov. Cienfuegos: Sierra de Trinidad, Dressler 1333 (holotype US).

Specimens studied: We did not find any specimens in the revised Cuban herbaria.

Lepanthes palpebralis Luer, Lindleyana 13: 143. 1998.* 
Type: Cuba: Holguín: Moa, camino al Toldo, La Calinga, Luer et al. 18658 (holotype HAJB!).

Lepanthes martae Luer, Selbyana 22(2): 109. 2001.

Type: Cuba: no other collection data available, $M$. A. Díaz 10 (holotype HAC).

Lepanthes pergracilis Schltr., in I.Urban, Symb. Antill. 9: 64. 1923. *

Type: Cuba: Prov. Santiago de Cuba: Sierra Maestra, Pico Turquino, E. L. Ekman 5490 (holotype S).

Specimens studied: We did not find any specimens in the reviewed Cuban herbaria.

Lepanthes silvae H.Dietr., Wiss. Z. Friedrich-SchillerUniv. Jena. Math-Naturwiss. Reihe 37(1): 157. 1988.*

Type: Cuba: Prov. Holguín, Mun. Moa; entre Revuelta de los Chinos y la base del Toldo, Alvarez et al. (holotype HAJB!: 56426 ).

Lepanthes minima-mundana Luer, Lindleyana 13: 142. 1998.

Type: Cuba: Holguín: Moa, camino al Toldo, Alto de La Calinga, Luer et al. 18661 (holotype HAJB!).

Lepanthes trichodactyla Lindl., Ann. Mag. Nat. Hist., series 3, 1: 329. 1858. *

Type: Cuba: Prov. Santiago de Cuba: Sierra Maestra, summit of Nemanima, C. Wright 661 (holotype K).

Lepanthes longicruris Schltr., in I.Urban, Symb. Antill. 9: 63. 1923.

Type: Cuba: Prov. Santiago de Cuba: Sierra Maestra, Loma del Gato, E. L. Ekman 6964 (holotype S).

SPECIMEN STUdied: HAC!: Wright 661.

Lepanthes turquinoensis Schltr., in I.Urban, Symb. Antill. 9: 65. 1923. *

Type: Cuba: Prov. Santiago de Cuba: Sierra Maestra, Pico Turquino, E. L. Ekman 5438 (holotype S).

SPeCimen STUdied: HAC!: Acuña 15076.

Lepanthes woodfredensis Luer, Lindleyana 13: 145. 1998.*

Type: Cuba: Holguín: Mayarí, Sierra de Nipe, Cayo Las Mujeres, Luer et al. 18623 (holotype HAJB!).

Lepanthes wrightii Rchb.f., Flora 48: 275. 1865.*

Type: Cuba: 1860-1864, C. Wright 3340 (holotype K).
Specimens studied: We did not find any specimens in the reviewed Cuban herbaria.

\section{Lepanthopsis (Cogniaux) Ames}

Lepanthopsis anthoctenium (Rchb.f.) Ames, Bot. Mus. Leafl. 1(9): 7. 1933.

Basionym: Pleurothallis anthoctenium Rchb.f., Linnaea 41: 94.1876.

Type: Haiti: Poiteau s.n. (holotype W).

Lepanthopsis domingensis Dod, Moscosoa 1: 44. 1977.

Type: Dominican Republic: Prov. La Vega, Jarabacoa, Río Baiguate, Dod 578 (holotype NY).

SPECIMENS STUDIED: HAC!: Alain 2749 ; Alain 7008.

Lepanthopsis melanantha (Rchb.f.) Ames, Bot. Mus. Leafl. 1(9): 19. 1933.

Basionym: Pleurothallis melanantha Rchb.f., Flora 48: 275.1865.

Type: Cuba: 1860-64, Wright 3342 (holotype K).

Pleurothallis floripicta Lindl. ex Griseb., Pl. Wright. 1: 219. 1860, nomen nudum.

Lepanthes harrisii Fawc. \& Rendle, J. Bot. 48: 5. 1909.

Type: Jamaica: Parish St. Catherine, Holly Mount, Mt. Diablo, Harris s.n. (holotype BM).

Lepanthes brevipetala Fawc. \& Rendle, J. Bot. 48: 5. 1909.

Type: Jamaica: Parish Portland, Silver Hill Woodland, Harris 7539 (BM).

Lepanthopsis quisqueyana Dod, Moscosoa 4: 155. 1986.

Type: Dominican Republic: Prov. Valverde, Cordillera Septentrional, Navarrete, Loma del Murazo, Dod 1186 (holotype JBSD).

Specimens studied: HAJB!: Ruperto 59621; HAC!: Alain et al. 7430; HUJB!: Urquiola et al. 5193; HOS!: Mújica 181.

Lepanthopsis microlepanthes (Griseb.) Ames, Bot. Mus. Leafl. 1(9): 24. 1933.

Basionym: Pleurothallis microlepanthes Griseb., F1. Brit. W. I. 610. 1864.

Trichosalpinx microlepanthes (Griseb.) Luer, Monogr. Syst. Bot. Missouri Bot. Gard. 15: 68. 1986.

Type: Jamaica: Parish Portland, Macfadyen et al. s.n. (holotype K). 
Lepanthes leonii C.Schweinf., in Leon, Fl. Cub. 1: 362. 1946.

Type: Cuba: Prov. Santiago de Cuba: Sierra Maestra, Loma del Gato, León, Clement \& Roca 10548 (holotype AMES).

SPecimens Studied: HAC!: Seen slide without data. Seen plants in flower in the Soroa Orchid Botanical Garden collected by Pérez et al., in March 11, 1998, in La Gran Piedra, Santiago de Cuba province.

Lepanthopsis pygmaea C.Schweinf., Amer. Orchid Soc. Bull. 14: 518. 1946.

Type: Haiti: Dept. Grand'Anse, Riviére Glace, Curtis 52 (holotype AMES).

Specimens STUdied: HAJB: Stenzel 632; HOS!: flowers in spirit: Mújica 126.

\section{LIPARIS L. C. Richard}

Liparis elata Lindl., Bot. Reg. 14: pl. 1175. 1828.

Type: Brazil: Rio de Janiero, Chamberlain s.n. (holotype K-L).

Liparis elata var. latifolia Ridley, J. Linn. Soc., Botany 22: 260. 1886.

Type: Cuba: Prov. Guantánamo, near Monte Verde, C. Wright 1495 (holotype K).

Liparis eggersii Rchb.f., Ber. Deutsch. Bot. Ges. 3: 278.1885.

Type; United States Virgin Islands: St. Thomas, near Bonne Resolution, Eggers s.n. (holotype $\mathrm{W})$.

Specimens Studied: HAC!: López 7012; HUJB!: Luis et al. 4158; HOS!: Padilla \& Alejandro 031; flowers in spirit: Bocourt et al. 094.

Liparis lindeniana (A. Rich. \& Galeotti) Hemsl., Gard. Chron. n.s. 11: 559. 1879.

Basionym: Malaxis lindeniana A.Rich. \& Galeotti, Ann. Sci. Nat. ser. 3,3: 18. 1845.

Type: "tab. 4, f. 1", probably based on Mexico, Terr. fl. blanches en fev., 1840, champs de terre chaude, Cordillera, Veracruz, Galeotti 5138 (P).

Liparis vexillifera (Lex.) Cogn. var. latifolia Cogn., Fl. Bras. 3(4): 289. 1895.

Type: Argentina: Sierra de Tucumán, Cuesta del

Gararatal, P. G. Lorentz \& G. Hieronymus 876 (syntype NY 290. 1895).

Liparis jamaicensis Lindl. ex Griseb., Cat. Pl. Cub. 261. 1866.

Sturmia elliptica Rchb.f., Linnaea 22: 833. 1849.
Liparis elliptica (Rchb.f.) Rchb.f., in W.G.Walpers, Ann. Bot. Syst. 6: 218. 1861, non Wight 1851.

Type: Guyana: Savanna Watu Ticaba, Schomburgk s.n. (lectotype K).

Liparis campestris Barb. Rodr., Gen. Sp. Orchid. 1: 36.1877.

Type: Brazil, Regnell s.n. (illustration by J. Barbosa Rodrigues, Inconografie des Orchidées du Brésil 2: t. 95B, RB).

Specimen Studied: HAC!: Grupo Humboldt 945.

This widespread species from tropical grasslands has been long confused with $L$. vexillifera (Lex.) Cogn., an entity restricted to seasonally cool and dry oak-coniferous forests in high mountains of Mexico, Guatemala, and El Salvador. Morphologically, L. lindeniana differs from L. vexillifera in the arcuate labellum, which is distinctly constricted above the middle and concave near the base (vs. an elliptic, straight labellum without a proximal concavity) (Salazar, pers. comm., 2015).

Liparis saundersiana Rchb.f., Gard. Chron. 1872: 1003. 1872.

Type: Jamaica: Collector unknown 2597 (holotype $\mathrm{W})$.

Specimens studied: Reported by Ackerman (2014) for Sierra de Nipe, province of Holguín and Sierra Maestra in Santiago de Cuba province. We did not find any specimens in the revised Cuban herbaria. Liparis viridipurpurea Griseb., Cat. Pl. Cub., 261. 1866.

Type: Cuba: Prov. Guantánamo, Mt. Toro, near Potosí, C. Wright 1691 (holotype GOET).

Liparis harrisii Fawc. \& Rendle, J. Bot. 46: 7. 1909.

Type: Jamaica: John Crow Peak, W. Harris 9786 (NY).

Liparis adamsii Proctor, J. Arnold Arbor. 63: 212. 1982.

Type: Jamaica: Trelawny: near Crown Lands, Proctor 34165 (holotype IJ).

SPECIMEN STUdied: HAC!: Alain 7028.

Macradenia R. Brown

Macradenia lutescens R.Br., Bot. Reg. 8: t. 612. 1822.

Type: Trinidad: hort. Griffith s.n. (lectotype [Nir, 2000, p. 231], illustration, Botanical Register 8: t. 612. 1822). 
Macradenia triandra Lindl., Edward's Bot. Reg.

21: t. 1815. 1836.

Type: Surinam: Lance s.n. (Illustration K-L).

Rhynchadenia cubensis A.Rich., in R.de la Sagra,

Hist. Fis. Cuba Bot. 11: 248, t. 85. 1850.

Type: Cuba (holotype P).

Specimens StUdied: HAJB!: Bisse et al. 40258; HOS!: Bocourt et al. 153; flowers in spirit: Bocourt et al. 096.

\section{Malaxis Solander ex Swartz}

Malaxis apiculata Dod, Moscosoa 4: 174. 1986.

Type: Dominican Republic: Río Limpio, Loma Nalga de Maco, Dod 1053 (holotype JBSD).

SPECimens Studied: We did not find any specimens in the reviewed Cuban herbaria.

Reported by Ackerman (2014) for the southern side of Pico Turquino (Seifriz 1031, US).

Malaxis hispaniolae (Schltr.) L.O.Williams, Caldasia 5: 14.1942.

BAsionym: Microstylis hispaniolae Schltr., Ark. Bot. 17 (7): 19. 1921.

Type: Haiti, Morne de la Hotte, Ekman 104 (holotype B, destroyed).

Specimens studied: We did not find any specimens in the reviewed Cuban herbaria. The Cuban record is based on Wright 1696 specimens at MO and BR which were previously determined as Malaxis spicata and M. umbelliflora, respectively (Ackerman, 2014).

Malaxis insularis (H.Dietr. \& M.Díaz) Nir, Orchid. Antill. 234. 2000**

Basionym: Malaxis domingensis subsp. insularis H.Dietr. \& M.Díaz, Feddes Repert. Spec. Nov. Regni Veg. 96: 560. 1985.

Type: Cuba: Isla de la Juventud, camino de Cayo Piedra a Punta del Este (holotype HAJB!: Alvarez et al. 45550).

Specimens studied: HAC!: Acuña 19633.

Malaxis labrosa (Rchb.f.) Acuña, Bol. Estación Exp. Agron. Santiago de las Vegas 56. 1939.*

Basionym: Microstylis labrosa Rchb.f., Flora 71: 151. 1888.

Type: Cuba: Potosi, C. Wright s.n. (holotype W). Microstylis confusa Cogn., in I.Urban, Symb. Antill. 6: 370. 1909.
Malaxis confusa (Cogn.) C.Schweinf., Bot. Mus. Leafl. 5: 104. 1938.

Type: Cuba: Orientali, 1856-1857, C. Wright 614 (holotype BR).

Specimens Studied: HAC!: León et al. 7036 and Pérez et al. 35528.

Malaxis spicata Sw., Prodr. Veg. Ind. Occ. 119. 1788. Microstylis spicata (Sw.) Lind., Gen. Sp. Orchid. P1. 19. 1830.

Type: Jamaica: Swartz s.n. (lectotype BM).

Microstylis floridana Chapm., Fl. S. U. S., ed. 1, 454. 1860.

Malaxis floridana (Chapm.) Kuntze, Revis. Gen. P1., pt. 2, 673. 1891.

Achroanthes floridana (Chapm.) Greene, Pittonia 2: 183.1891

Type: Florida, Chapman s.n. (NY?).

Malaxis pusilla Ames \& C.Schweinf., Sched. Orchid. 8: 10. 1925.

Type: Cuba: southern Oriente and Pico Turquino, Sierra Maestra, Leon 10762 (holotype NY).

Malaxis brittonii Acuña, Bol. Estación Exp. Agron.

Santiago de las Vegas 57. 1939.

Type: Cuba: Camagüey, Caobilla, Finca La Ciega, Roig 4026 (holotype HAC?, HAJB?).

Specimens studied: HAC!: León et al. 60438; HUJB!: Luis et al. 4046; HOS!: Mújica et al. 190; flowers in spirit: Mújica et al. 057.

Malaxis umbelliflora Sw., Prodr. Veg. Ind. Occ. 119. 1788.

Basionym: Malaxis umbellulata Sw., Fl. Ind. Occid. 3: 1444. 1806.

Microstylis umbellulata (Sw.) Lindl., Gen. Sp. Orchid. Pl. 19. 1830.

Microstylis umbelliflora (Sw.) Hitch., Ann. Missouri Bot. Gard. 4: 132. 1893.

Type: Jamaica, O. Swartz s.n. (BM).

Specimens studied: HAJB!: Bisse et al. 40599.

Malaxis unifolia Michx., Fl. Bor. Amer. 157. 1803.

Microstylis unifolia (Michx.) Britton, Sterns \& Poggenb., Prelim. Cat. 51.1888.

Type: Carolina, Swamps de la Balle, Michxaux s.n. (lectotype P).

Malaxis ophioglossoides Muhl. ex Willd., Sp. P1., ed. 4, 4: 90. 1805.

Achroanthes ophioglossoides (Muhl. ex Willd.) Raf., Med. Repos., ed. 2, 5: 352. 1808. 
Type: Muller 173 (lectotype PH).

SPECIMENS STUdied: HAJB!: Dietrich et al. 50836; HAC!: Alain 4696.

\section{MaXillaria Ruiz \& Pavón}

Maxillaria adendrobium (Rchb.f.) Dressler, Taxon 13: 248. 1964.

BASIONYM: Ponera adendrobium Rchb.f., Flora 48: 278. 1865.

Pleuranthium adendrobium (Rchb.f.) Benth. \& Hook., J. Linn. Soc., Botany 18: 312. 1881.

Neourbania adendrobium (Rchb.f.) Fawc. \& Rendle, J. Bot. 47: 125. 1909.

Ornithidium adendrobium (Rchb.f.) Blanco \& Ojeda, Lankesteriana 10: 532. 2007.

Type: Cuba: Wright 1697 (holotype K).

SPecimen Studied: HAJB!: Bisse et al. 52937.

Maxillaria alba (Hook.) Lindl., Gen. Sp. Orchid. P1. 143. 1832.

Basionym: Dendrobium album Hook., Exot. Fl. 2: t. 142.1825.

Broughtonia alba (Hook.) Spreng., Syst. Veg. 3: 735. 1826.

Camaridium album (Hook.) Hoehne, Arq. Bot. Estado Sao Paulo, n. s., 2: 72. 1947.

Maxillariella alba (Hook.) M.A.Blanco \& Carnevali, Lankesteriana 7: 528. 2007.

Type: Jamaica: Wiles ex Shepherd s.n. (holotype $\mathrm{K})$.

SPecimen StUdied: HAJB!: Bisse et al. 49670.

Maxillaria conferta (Griseb.) C.Schweinf. ex León, Contr. Ocas. Mus. Hist. Nat. Colegio "De La Salle" 8: 395. 1946.

Ornithidium confertum Griseb., Fl. Britt. W. I. 626. 1864, nomem illegitimum, cited by the earlier name.

Camaridium vestitum (Sw.) Lindl., Fl. Brit. W. I. 626. 1864.

Type: Cuba, Wright 650 (lectotype USF).

Scaphyglottis parviflora Poepp. \& Endl., Nov. Gen. Sp. Pl. 1: 58, t. 1836.

Maxillaria purpurea (Spreng.) Ames \& Correll, Bot. Mus. Leafl. 11: 16. 1943.

Maxillaria parviflora (Poepp. \& Endl.) Garay, Bot. Mus. Leafl. 21: 258. 1967.

Type: Peru: Cuchero: Poeppig s.n. (holotype W!), non Camaridium parviflorum Fawcett.
Camaridium micranthum M.A.Blanco, Lankesteriana 7: 520. 2007.

Type: Brazil: Forests of the Serra do Mar near Palmeiras (holotype Iconografia des orchidées du Bresil 6: pl. 298A).

Specimens Studied: HAC!: León 7138; HUJB!: Luis et al. 4060.

Maxillaria crassifolia (Lindl.) Rchb.f., Bonplandia 2: 16. 1854.

Basionym: Heterotaxis crassifolia Lindl., Bot. Reg. 12: t. 1028. 1826.

Dicrypta crassifolia (Lindl.) Loudon, Suppl. Hort. Brit. Suppl. 3: 536. 1839.

Type: Jamaica, J. Lee s.n. (holotype K).

Epidendrum sessile Sw., Prodr. Veg. Ind. Occ. 122. 1788.

Maxillaria sessilis (Sw.) Fawc. \& Rendle, Fl. Jamaica 1: 120. 1910, not Maxillaria sessilis Lindl., 1845.

Heterotaxis sessilis (Sw.) F.Barros, Hoehnea 29: 112. 2002.

Type: Jamaica (lectotype BM).

SPeCimens STUdied: HUJB!: Luis et al. 4298; HOS!: Mújica \& Elaine 212; flowers in spirit: Mújica \& Elaine 070.

Maxillaria grisebachiana Nir \& Dod, Orchid. Antill. 243. 2000.

Camaridium grisebachianum (Nir \& Dod) M.A.Blanco, Lankesteriana 7(3): 520. 2007.

Type: Haiti: Dept. Nord-Ouest, Massif du Nord, Port-de-Paix, Haut Piton, Ekman H4609, (holotype NY).

Specimen studied: HAJB!: Bisse \& Díaz 37130.

Maxillaria pudica Carnevali \& J.L.Tapia, Brittonia 54: 463. 2001.

Mormolyca pudica (Carnevali \& J.L.Tapia) M.A.Blanco, Lankesteriana 7: 531. 2007.

Type: Jamaica: Parish Portland, Mabess River, Harris 7615 (holotype BM).

Maxillaria rufescens var. minor Fawc. \& Rendle, J. Bot. 48: 108. 1910.

Type: Jamaica: Parish Clarendon, Mt. Moses, J.

P. (Jamaica Plants) 2009; Syme s.n.; Parish Portland, Mabess River, Harris s.n.).

SPeCIMENS StUdied: HAJB!: Bisse \& Dietrich 46234; HAC!: López Figueiras 7072; HOS!: flowers in spirit: Pérez \& Bocourt 075. 
Maxillaria valenzuelana (A.Rich.) Nash, Bull. Torrey Bot. Club 34: 121. 1907.

Basionym: Pleurothallis valenzuelana A.Rich., in R.de la Sagra, Hist. Fis. Cuba Bot. 11: 234. 1850.

Marsupiaria valenzuelana (A.Rich.) Garay, Arq. Jard. Bot. Río de Janeiro 12: 183. 1952.

Heterotaxis valenzuelana (A.Rich.) Ojeda \& Carnevali, Novon 15: 581. 2005.

Type: Cuba, J. M. Valenzuela s.n. (holotype P). Maxillaria valenzuelana subsp. angustata J.T.Atwood, Ic. Plant. Trop. 1373. 1989.

Maxillaria valenzuelana var. angustata (J.T.Atwood) Senghas, Schltr., Orchideen ed. 3, 1/B (29): 1779. 1994.

Type: Nicaragua: Zelaya, Siuna, F. Ortiz 612 (holotype SEL).

Specimens StUdied: HUJB!: Luis et al. 4068 \& 4068-A; HOS!: Martínez 138; flowers in spirit: Martínez 069.

\section{Mesadenus Schlechter}

Mesadenus lucayanus (Britton) Schltr., Beih. Bot. Centralbl., Abt. 2, 37: 368. 1920.

Basionym: Ibidium lucayanum Britton, Bull. New York Bot. Gard. 5: 312. 1907.

Spiranthes lucayana (Britton) Cogn., in I.Urban, Symb. Antill. 6: 238. 1909.

Type: Bahamas: Great Exuma, near Rollstown, Britton \& Millspaugh 3077 (holotype NY).

Spiranthes stahlii Cogn., in I.Urban, Symb. Antill. 6: 341. 1910.

Mesadenus stahlii (Cogn.) Garay, Bot. Mus. Leafl. 28: 336. 1982.

Type: Puerto Rico: near Cangrejos, Santurce, Stahl 1010 (holotype BR).

SPECIMENS STUDIED: HAJB!: Leiva 55425; HUCLV!: Castañeda et al. 3097.

\section{Microchilus Presley}

Microchilus familiaris Ormerod, Harvard Pap. Bot. 14: 121. 2009.

Type: Cuba, Oriente, Sierra Maestra, Río Oro, E. L. Ekman 5103 (holotype, S).

SPecimens STUdied: We did not find any specimens in the reviewed Cuban herbaria. Until recently this species has languished in folders of Microchillus hirtellus. Both species occurs in the same habitats and at times may be sympatric. Reported for Sierra Maestra, Santiago de Cuba y Macizo SaguaBaracoa in Guantánamo province (Ackerman, 2014).

Microchilus hirtellus (Sw.) D.Dietr., Syn. Pl. 5: 166. 1852 .

Basionym: Satyrium hirtellum Sw., Prodr. Veg. Ind. Occ. 118. 1788.

Orchis hirtella Sw., Vet. Handl. Stockh. 21: 207. 1800.

Habenaria hirtella (Sw.) Spreng., Syst. Veg. 3: 690. 1826.

Physurus hirtellus (Sw.) Lindl., Gen. Sp. Orchid. P1. 501. 1840.

Erythrodes hirtella (Sw.) Fawc. \& Rendle, Fl. Jamaica 1: 29. 1910.

Type: Jamaica, O. Swartz s.n. (lectotype BM).

Specimens STUdied: HAJB!: Bisse et al. 52482; HOS!: Mújica et al. 218; flowers in spirit: Mújica et al. 103.

Microchilus plantagineus (L.) D.Dietr., Syn. P1. 5: 166. 1852.

BAsionym: Satyrium plantagineum L., Syst. Nat., ed. 10, 2: 1244. Oct. 1759.

Orchis plantaginea (L.) Sw., Kongl. Vetensk. Acad. Handl. 21: 207. 1800.

Physurus plantagineus (L.) Lindl., Gen. Sp. Orchid. Pl. 503. 1840.

Erythrodes plantaginea (L.) Fawc. \& Rendle, Fl. Jamaica 1: 28. 1910.

Type: "Orchis elatior latifolia, asphodeli radice, spica strigosa" in Sloane, $A$ voyage to the islands Madera, Barbados, Nieves, $S$. Christophers and Jamaica 1: t. 147, f. 2.1707 (lectotype, Cribb in Cafferty and Jarvis, 1999, p. 49).

SPeCimens StUdied: HUJB!: Urquiola et al. 5189 \& 5189-A; HOS!: Urquiola et al. 016; flowers in spirit: Pérez et al. 102.

\section{Nidema Britton \& Millspaugh}

Nidema ottonis (Rchb.f.) Britton \& Millsp., Bahama Fl. 94. 1920.

BASIONYM: Epidendrum ottonis Rchb.f., Hamb. Gartenz. 14: 213. 1858.

Type: Venezuela: Caracas, Otto 1011 (holotype W). 
Nidema boothii var. triandra Schltr., Repert. Spec.

Nov. Regni Veg. 19: 211. 1923.

Type: Panama: foothills east of city, C. W. Powell 131 (lectotype AMES).

Nidema boothii var. australis Schltr., Repert. Spec.

Nov. Regni Veg. 19: 221. 1923.

Type: Costa Rica: San Pedro de San Ramón, Brenes 158 (holotype $\mathrm{B}$, destroyed).

SPECIMENS STUDIED: HAJB!: Bisse et al. 28822; HUCLV!: Sotomayor 4669; HOS!: (not registered): collector unknown 226; flowers in spirit: collector unknown 159.

\section{OCTOMERIA R. Brown}

Octomeria ventii H.Dietr., Fl. Rep. Cuba. Ser. A., 12(2): 17. 2007. *

Type: Cuba: Prov. Guantánamo, Monteverde, López Figueiras 2559 (holotype HAJB!).

\section{Oeceoclades Lindley}

Oeceoclades maculata (Lindl.) Lindl., Gen. Sp. Orch. P1. 237. 1833.

Basionym: Angraecum maculatum Lindl., Coll. Bot. t. 15. 1821.

Aerobium maculatum (Lindl.) Spreng., Syst. Veg. 3: 718.1826.

Eulophidium maculatum (Lindl.) Pfitzer, Entwurf. Anordn. Orch. 88. 1887.

Type: Brazil: ex Hort. Loddiges (holotype destroyed; lectotype K).

Specimen studied: HOS!: Mújica 165.

\section{Oncidium Swartz}

Oncidium ensatum Lindl., Edward's Bot. Reg. 28 (Misc.) 17. 1842.

Type: Guatemala, hort. Loddiges s.n. (holotype $\mathrm{K}-\mathrm{L}$ ).

Cyrtopodium verrucosum Griseb., Cat. Pl. Cub. 265. 1866.

Type: Cuba: 1860-1864, Wright 3324 (holotype GOET).

Oncidium floridanum Ames, Schedul. Orchid. 7: 13. 1924.

Type: United States of America: Florida, Dade County, Black Point Creek, A. A. Eaton 957 (holotype AMES).
Specimens Studied: HAJB!: Bisse et al. 32780-A; HOS!: flowers in spirit: collector unknown 012.

\section{Orthochilus Hochst. ex A.Richard}

Orthochilus ecristatus (Fernald) Bytebier, Taxon 63: 18. 2014.

Basionym: Cyrtopodium ecristatum Fernald, Bot. Gaz. (Crawfordsville) 24: 433. 1887.

Cyrtopodium strictum Griseb., Cat. Pl. Cub. 266. 1866.

Triorchos ecristatus (Fernald) Small, Fl. S.E. U.S. 329. 1903.

Eulophia ecristata (Fernald) Ames, Contr. Knowl. Orch. S. Fl. 19. 1904.

Pteroglossaspis ecristata (Fernald) Rolfe, Orch. Rev.12: 136. 1904.

Type: United States of America: Florida: Duval, near Jacksonville and borders of Indian River, A. H. Curtis 2808 (syntypes AMES).

SPECIMENS STUdied: HUJB!: Urquiola et al. 5509.

The recent molecular phylogenetic analysis of plastid and nuclear DNA sequences by Martos et al. (2014), showed that, as traditionally circumscribed, Eulophia is paraphyletic and consists of two groups of species. One of such groups consists of Eulophia s.s., which includes the type species of the genus (E. guineensis Lindl.) and is sister to Acrolophia Pfitzer, with Oeceoclades Lindl. in turn being sister to them. The other group is sister to the whole above clade, to which the name Orthochilus Hochst. ex A.Rich. was applied by Martos et al. (2014), includes the species formerly known as Eulophia ecristata. Chase et al. (2015) considered the results of the study of Martos et al. (2014) inconclusive because of insufficient taxonomic sampling, and did not recognize Orthochilus. Nevertheless, accepting both Acrolophia and Oeceoclades as distinct genera while including the Orthochilus clade in Eulophia is inconsistent, since these two groups are not sisters of each other. Hence, we prefer to accept Martos et al. (2014) view, accepting Orthochilus and including in it the former E. ecristata.

\section{Pelexia Poiteau ex Lindley}

Pelexia adnata (Sw.) Spreng., Syst. Veg. 3: 704. 1826. Basionym: Satyrium adnatum Sw., Prodr. Veg. Ind. Occ. 118. 1788.

Neottia adnata (Sw.) Sw., Fl. Ind. Occid. 3: 1409. 1805. 
Spiranthes adnata (Sw.) León, Contr. Ocas. Mus. Hist. Nat. Colegio "La Salle" No.8, 358. 1946.

Type: Jamaica: Parish Clarendon, May Pen, O.Swartz s.n. (lectotype UPS).

Pelexia spiranthoides Lindl., Bot. Reg. 12: t. 985. 1826.

Type: St. Vincent 1825, McRae s.n. (holotype K-L).

Pelexia stenorrhynchoides Griseb., Cat. P1. Cub. 269. 1886.

Spiranthes stenorrhynchoides (Griseb.) León, Fl. Cub. 357. 1946.

Type: Cuba: Occidentale (?), C. Wright s.n. (holotype unknown).

Specimens StUdied: HAC!: Acuña 11159; HOS!: Bocourt et al. 160; flowers in spirit: Bocourt et al. 091.

Pelexia maxonii Ames, Proc. Biol. Soc. Wash. 35: 85. 1922. *

Type: Cuba: Prov. Guantánamo, Yateras, southeast of Jaguey, Maxon 4437 (holotype US).

Specimens StUdied: We did not find any specimens in the reviewed Cuban herbaria.

\section{Phaius Loureiro}

Phaius tancarvilleae (Banks ex L'Héritier) Blume, Mus. Bot. Lugduno-Batar., 2:177. 1852.

Basionym: Limodorum tancarvilleae Banks ex L'Héritier, Sert. Angl. 28. 1788.

Bletia tancarvilleae (Banks ex L'Héritier) R.Br., in W.T.Aiton, Hort. Kew., ed. 2, 5: 207. 1813.

Type: China: hort. Tankerville s.n. (holotype BM). Specimens STUdied: HAJB!: Bisse et al. 13560; HOS!: Elaine et al. 242; flowers in spirit: Martinez 010.

This is an introduced species from the Pacific islands that has escaped from cultivation and has become a weed in some places in Cuba.

Phloeophila Hoehne \& Schlechter

Phloeophila nummularia (Rchb.f.) Garay, Orquideología 9: 118. 1974.

BAsionym: Pleurothallis nummularia Rchb.f., Flora 48: 276. 1866.

Specklinia nummularia (Rchb.f.) Luer, Monogr. Syst. Bot. Missouri Bot. Gard. 95: 262. 2004.
Type: Cuba: Prov. Guantánamo, near Monte Verde, C. Wright 1513 (holotype K).

Specimen STUdied: HAC!: Alain 7201.

Phloeophila oricola (H.Stenzel) Luer, Monogr. Syst. Bot. Missouri Bot. Gard. 105: 193. 2006. *

Basionym: Pleurothallis oricola Stenzel, Willdenowia 32: 99. 2002.

Type: Cuba: Pinar del Río: Guanahacabibes Peninsula, near María La Gorda, Urquiola et al. s.n. (holotype HAJB).

SPecimen studied: HOS!: Mújica et al 162.

Platanthera L. C. Richard

Platanthera replicata (A.Rich.) Ackerman, Lindleyana 12: 151. 1997. *

BAsionym: Habenaria replicata A.Rich., in R.de la Sagra, Hist. Fis. Cuba, Bot. 11: 250, t. 86. 1850.

Type: Cuba: Sagra s.n. (holotype P).

Specimens studied: We did not find any specimens in the reviewed Cuban herbaria. A population of this species has been seen in flower by the authors in San Ubaldo, near Cortés, municipality of Guane, Pinar del Río province.

\section{Platystele Schlechter}

Platystele hyalina H.Stenzel, Willdenovia 32: 103. 2002. *

Type: Cuba: Prov. Holguín, Sierra de Moa, El Toldo, H. Stenzel 569 (holotype HAJB!).

Platystele ovalifolia (H.Focke) Garay \& Dunst., Venez. Orchids III. 2: 268. 1961.

Basionym: Stelis ovalifolia H.Focke, Tijdschr. Natuurk. Wetensch. Kunsten 2: 202, 1849.

Pleurothallis ovalifolia (H.Focke) Rchb.f., in W.G.Walpers, Ann. Bot. Syst. 6: 188. 1861.

Type: Guyana: Aruka, E. F. Im Thurn 36 (K).

Pleurothallis rhomboglossa Rchb.f., Flora 48: 276. 1865.

Type: Cuba: Prov. Guantánamo, near Monte Verde, Wright 1506 (holotype K).

SPeCIMEN STUdiEd: HAJB!: Alain \& Figueiras 7316.

\section{Platythelys Garay}

Platythelys querceticola (Lindl.) Garay, Bradea 2: 197. 1977. 
Basionym: Physurus querceticola Lindl., Gen. Sp. Orchid. P1. 505. 1840.

Goodyera querceticola Chapm., Fl. S.E. U.S. 463. 1860.

Erythrodes querceticola (Lindl.) Ames, Orchidaceae 5: 29. 1915.

Type: United States of America: Louisiana, near New Orleans, Ingalls s.n. (holotype K-L).

Physurus sagraeanus A.Rich., in R.de la Sagra, Hist. Fis. Cuba, Bot. 11: 253, pl. 88. 1850.

Erythrodes sagraeana (A.Rich.) Ames, Orchidaceae fasc. 5: 29. 1915.

Platythelys sagraeana (A.Rich.) Garay, Bradea 2: 197. 1977.

Type: Cuba: Sagra s.n. (holotype P).

Specimens studied: HUJB!: Luis et al. 3050 \& 3050-A; HOS!: Mújica et al. 184; flowers in spirit:

Pérez et al. 098.

The genus Platythelys Garay was synonymized with Aspidogyne Garay by Meneguzzo (2012), who argued that the distinguishing characters used by Garay (1978) to separate the genera are inconsistent, and such approach was followed also by Ormerod (2013) and Chase et al. (2015). Nevertheless, ongoing molecular phylogenetic studies of New World Goodyerinae (G. A. Salazar et al., unpubl. manuscript) indicates that relationships in this group are more complex than casual floral comparisons suggests and taxonomic changes should wait until a clear phylogenetic context is available. Hence, we prefer to be conservative and maintain Platythelys.

\section{Pleurothallis R. Brown}

Pleurothallis appendiculata Cogn., in I.Urban, Symb. Antill. 7: 174. 1912.

Antilla appendiculata (Cogn.) Luer, Monogr. Syst. Bot. Missouri Bot. Gard. 95: 255. 2004.

Type: Dominican Republic: Prov. La Vega: shady forest near Constanza, H. von Türckheim 3233 (holotype BR).

SPECIMEN STUdied: HAJB!: Alvarez et al. 63698.

Pleurothallis caymanensis C.D.Adams, Orquideología 6: 146. 1971.

Proctoria caymanensis (C.D.Adams) Luer, Monogr. Syst. Bot. Missouri Bot. Gard. 95: 258. 2004.
Type: Cayman Islands: Grand Cayman, southeast of Old Man Village, G.R. Proctor 27983 (holotype IJ).

SPeCimens StUdied: HUJB!: Urquiola et al. s.n.; HOS!: Pérez et al. 017; flowers in spirit: Pérez et al. 066 .

Pleurothallis denticulata Cogn., in I.Urban, Symb. Antill. 6: 425. 1909.

Specklinia denticulata (Cogn.) Luer, Monogr. Syst. Bot. Missouri Bot. Gard. 95: 260. 2004.

Type: Cuba: Prov. Guantánamo, near Monte Verde, peak of Loma del Gato, C. Wright 657 (holotype BR 843468).

Pleurothallis parvula Ames \& C.Schweinf., Schedul. Orchid. 8: 30. 1925.

Antilla parvula (Ames \& C.Schweinf.) Luer, Monogr. Syst. Bot. Missouri Bot. Gard. 95: 255. 2004.

Type: Cuba: Santiago de Cuba: Sierra Maestra, Pico Turquino, Alta Maestra, Bro. Leon 10790 (holotype NY 59805).

Specimens studied: HAJB!: Dietrich et al. 63434; HAC!: Acuña 21107; HOS!: flowers in spirit: Pérez \& Bocourt 039.

Pleurothallis ekmanii Schltr., in I.Urban, Symb. Antill. 9: 61. 1923. *

Atopoglossum ekmanii (Schltr.) Luer, Monogr. Syst. Bot. Missouri Bot. Gard. 95: 255. 2004.

Type: Holguín: Sierra de Cristal, E. L. Ekman 6831 (holotype S).

Pleurothallis bovilabia C.Schweinf., Amer. Orchid Soc. Bull. 15: 235. 1946.

Type: Cuba: Holguín, Moa, Monte de la Breña, Léon et al. 22586 (holotype AMES).

Specimens studied: HAJB!: Alvarez et al. 56273; HAC!: Clement 7183.

Pleurothallis excentrica (Luer) Luer, Revista Soc. Boliv. Bot. 3: 50. 2001. *

BASIONym: Octomeria excentrica Luer, Lindleyana 14: 106. 1999.

Atopoglossum excentricum (Luer) Luer, Monogr. Syst. Bot. Missouri Bot. Gard. 95: 255. 2004.

Type: Cuba: Holguín, Moa, Río Cayoguan, Alain et al. 896 (holotype AMES).

Specimen STUdied: HAJB!: Genes et al. 58495.

Pleurothallis prostrata Lindl., Ann. Mag. Nat. Hist., ser. $3,1: 327.1858$. * 
Humboltia prostrata (Lindl.) Kuntze, Revis. Gen. P1. 2: 668. 1891.

Octomeria prostrata H.Stenzel, Lindleyana 16: 26. 2001.

Antilla prostrata (Lindl.) Luer, Monogr. Syst. Bot. Missouri Bot. Gard. 95: 255. 2004.

Atopoglossum prostratum (H.Stenzel) Luer, Monogr. Syst. Bot. Missouri Bot. Gard. 95: 255. 2004.

Type: Cuba: Holguín, Sierra de Cristal, Pico Cristal, Río Levisa, Stenzel 516 (holotype HAJB!).

Pleurothallis pruinosa Lindl., Edward's Bot. Reg. 28 (Misc.): 75. 1842.

Humboltia pruinosa (Lindl.) Kuntze, Revis. Gen. Pl. 668. 1891.

Type: Surinam: F. L. Splitgerber 527 (holotype $\mathrm{K}-\mathrm{L}$ ),

Stelis flavida H.Focke, Tijdschr. Natuurk. Wetensch. Kunsten 2: 202. 1849.

Pleurothallis flavida (H.Focke) Lindl., Fol. Orchid., 9: 44. 1859.

Type: Surinam, H. C. Focke 55 (holotype W).

Pleurothallis brachyglottis Rchb.f., Flora 48: 275. 1865.

Type: Cuba: Oriente, C. Wright 3344 (holotype K). Specimens studied: HAJB!: Bisse et al. 37122; HAC!: Alain et al. 5470; HOS!: collector unknown 060; flowers in spirit: collector unknown 078.

Pleurothallis quadrifida (Lex.) Lindl., Edward's Bot. Reg. 28 (Misc.): 70.1842.

Basionym: Dendrobium quadrifidum Lex., in P.de la Llave \& J.M. de Lexarza, Nov. Veg. Descr. 2 (Orchid. Opusc.) 40. 1825.

Humboltia quadrifida (Lex.) Kuntze, Revis. Gen. P1. 2: 668. 1891.

Stelis quadrifida (Lex.) Solano \& Soto Arenas, Icon. Orchid. 5-6: 11. 2003.

Specklinia quadrifida (Lex.) Luer, Monogr. Syst. Bot. Missouri Bot. Gard. 95: 263. 2004.

Loddigesia quadrifida (Lex.) Luer, Monogr. Syst. Bot. Missouri Bot. Gard. 105: 251. 2006.

Type: Mexico, La Llave \& Lexarza s.n. (holotype $\mathrm{G})$.

Pleurothallis racemiflora Lindl. ex Lodd., Bot. Cab. 10: t. 949. 1825, non P. racemiflora (Sw.) Lindl. ex Hook., 1824.
Stelis racemiflora Lodd. ex W.H.Baxter, in J.C.Loudon, Hort. Britton, Suppl. 3: 643. 1850. Type: Jamaica: Loddiges s.n. (holotype K).

Specimen STUdied: HAC!: Morton \& Acuña 12328. Pleurothallis ruscifolia (Jacq.) R.Br., in W.T.Aiton, Hortus Kew. 5: 211. 1813.

BAsionym: Epidendrum ruscifolium Jacq., Enum. Syst. P1. 29. 1760.

Dendrobium ruscifolium (Jacq.) Sw., Nova Acta Regiae Soc. Sci. Upsal. 6: 84. 1799.

Humboltia ruscifolia (Jacq.) Kuntze, Revis. Gen. P1. 668. 1891.

Type: Martinique: Plumier s.n. (lectotype Plumier, Plantarum Americanarum pl. 176, f. 2. 1758).

Specimens studied: HAJB!: Bisse et al. 52185; HAC!: Morton \& Acuña 12327.

Pleurothallis trichophora Lindl., Ann. Mag. Nat. Hist., ser. 3, 1: 326. 1858. *

Antilla trichophora (Lindl.) Luer, Monogr. Syst. Bot. Missouri Bot. Gard. 95: 255. 2004.

Type: Cuba: Oriente, 1856-57, Loma del Gato, $C$. Wright 659 (holotype K-L).

Specimens STUdied: HAJB!: Arias et al. 63208; HAC!: Clemente 7251.

\section{Polystachya Hooker}

Polystachya concreta (Jacq.) Garay \& H.R.Sweet, Orquideologia 9. 206. 1974.

BAsionym: Epidendrum concretum Jacq., Enum. Syst. P1. 30. 1760.

Type: Martinique, Jacquin s.n. (holotype W).

Epidendrum minutum Aubl., Hist. Pl. Guiane 2: 824. 1775.

Polystachya minuta (Aubl.) Britton, in J.Small, Fl. S.E. U.S. 328. 1903

Type: Martinique, Plumier s.n. (holotype original illustration at $\mathrm{P})$.

Polystachya cubensis Schltr., in I.Urban, Symb. Antill. 9: 59. 1923.

Type: Cuba: Prov. Holguín, Sierra de Nipe, Camino Real, Ekman 9987 (holotype B, destroyed).

SPeCimens STUdied: HUJB!: Urquiola et al. 1149; HUCLV!: Noa \& Sotomayor 2458; HOS!: Félix 131; flowers in spirit: Félix 056.

Polystachya foliosa (Hook.) Rchb.f., in W.G.Walpers, Ann. Bot. Syst. 6: 640. 1863. 
Basionym: Stelis foliosa Hook., Ann. Mag. Nat. Hist. 2: 330. 1839.

Dendrorchis foliosa (Hook.) Kuntze, Revis. Gen. Pl. 2: 658. 1891.

Type: Guyana, C. S. Parker s.n. (holotype cited for K, by Garay \& H.R.Sweet (1974)).

Polystachya cerea Lindl., Edward's Bot. Reg. 26 (Misc.): 86, no. 208. 1840.

Type: Mexico: Oaxaca, flowered in cultivation, Loddiges s.n. (holotype K-L).

Polystachya minor Fawc. \& Rendle, J. Bot. 48: 106. 1910.

Type: Jamaica: Parish Manchester, Lancaster, Harris 7777 (holotype BM).

Polystachya foliosa var. triandra Sauleda \& R.M.Adams, Brittonia 31: 294. 1979.

Type: Bahama Islands: Andros, Owens Town, Sauleda et al. 2105 (holotype NY ).

Specimen STUdied: HAJB!: Alvarez et al. 54974.

A plant initially discovered by R. Pérez in 2002 in Topes de Collantes, Scanti Spíritus province, might represent an undescribed species of Polystachya. Flowers in spirit are conserved in the Soroa Orchid Botanical Garden Herbarium. It is vegatively similar to $P$. concreta but the flowers are easily distinguished by the yellowish-red color and the smaller size.

\section{Ponthieva R. Brown}

Ponthieva brittoniae Ames, Torreya 10: 90. 1910.

Type: Bahamas: New Providence, Maidenhead Coppice, E. G. Britton 3297 (holotype NY).

Ponthieva ekmanii Mansf., Ark. Bot. 22A (17): 10. 1929.

Ponthieva racemosa var. brittoniae (Ames) Luer, Native Orchids Florida 82. 1972.

Type: Haiti: Tortue Island, above Source Dupuy, Ekman H 9730 (holotype B, destroyed).

Ponthieva parvula Ames \& C.Schweinf., Bot. Mus. Lafl. 4: 39-40. 1936.

Type: Mexico, Yucatán, Tuxpeña, C.L. Lundell 1213 (MICH).

Ponthieva poitaei Rchb.f. ex Nir, Orchid. Antill. 312. 2000.

Type: "Santo Domingo" (Haiti): Poiteau s.n. (holotype W).

SPeCimen STUdied: HAC!: Acuña 15080.
Ponthieva diptera Lindl. \& Rchb.f., Bonplandia 2: 278. 1854.

Type: Colombia: Santa María, Schlim 987 (holotype W).

Ponthieva dicliptera Rchb.f., Flora 69: 548. 1886.

Type: Colombia: Lehmann 1860 (holotype W).

Ponthieva harrisii Cogn., Repert. Spec. Nov. Regni Veg. 6: 304. 1909.

Type: Jamaica: Parish Portland, Mabess, Harris 7883 (holotype BR).

Ponthieva elata Schltr., Spec. Nov. Regni Veg. Beih. 7: 63. 1920.

Type: Colombia: Cauca, Madero 169 (holotype B). Specimens studied: HAC!: León 11002.

Ponthieva pauciflora (Sw.) Fawc. \& Rendle, Fl. Jamaica 1: 38. 1910.

Basionym: Cranichis pauciflora Sw., Fl. Ind. Occid. 3: 1434. 1806.

Type: Jamaica: O. Swartz S.n. (type ?).

Ponthieva haitiensis Mansf., Ark. Bot. 20A (15): 14. 1926.

Type: Haiti: Dept. Ouest, Morne Tranchant, Ekman H1181 (holotype S).

SPecimen STUdied: HAC!: Acuña 20017.

Ponthieva racemosa (Walter) C.Mohr, Contr. U.S. Natl. Herb. 6: 460. 1901.

Basionym: Arethusa racemosa Walter, Fl. Carol. 222. 1788 .

Type: T. Walter s.n., United States of America: Carolina (photo at BM).

Neottia glandulosa Sims, Bot. Mag. 22: t. 842. 1804. Ponthieva glandulosa (Sims) R.Br., in W.T.Aiton, Hortus Kew., ed. 2, 5: 200. 1813.

Type: United States of America: Virginia, Clayton 318 (holotype BM).

Ponthieva lancifolia A.Rich., in R.de la Sagra, Hist. Fis. Cuba, Bot. 11: 251. 1850.

Type: Cuba: Sagra s.n. (holotype P).

Specimens studied: HUJB!: Ferro et al. 4324; HUCLV!: Noa et al. 782.

Ponthieva ventricosa (Griseb.) Fawc. \& Rendle, Fl. Jamaica 1: 39. 1910.

Basionym: Cranichis ventricosa Griseb., Fl. Brit. W. I. 639. 1864.

Type: Jamaica: Wilson s.n. (holotype K).

Specimens studied: HAC!: León 7321; HOS!: Pérez et al. 168; flowers in spirit: Pérez et al. 097. 


\section{Prescottia Lindley ex Hooker}

Prescottia oligantha (Sw.) Lindl., Gen. Sp. Orchid. Pl. 454. 1840.

Basionym: Cranichis oligantha Sw., Prodr. Veg. Ind. Occ. 120. 1788.

Cranichis micrantha Spreng., Syst. Veg., ed. 16, 3: 700.1826.

Type: Jamaica: Mont. Caerul. Blue Mountains, $O$. Swartz s.n. (lectotype BM).

Specimens Studied: HAC!: Acuña 27138; HOS!: Pérez et al. 090.

Prescottia stachyodes (Sw.) Lindl., Edward's Bot. Reg. 22: sub t. 1915.

Basionym: Cranichis stachyodes Sw., Prodr. Veg. Ind. Occ. 120. 1788.

Type: Jamaica: Blue Mountains, O. Swartz s.n. (lectotype BM).

Prescottia pellucida Lindl., Ann. Mag. Nat. Hist., ser. III, 1: 335. 1858.

Type: Cuba: Prov. Santiago de Cuba, Loma del

Gato, C. Wright s.n. (holotype K-L).

Specimens STUdied: HAJB!: Bisse 1562; HUCLV!: Noa et al. 1443; HOS!: Elaine et al. 244; flowers in spirit: Pérez et al. 100.

Prosthechea Knowles \& Westcott

Prosthechea boothiana (Lindl.) W.E.Higgins, Phytologia 82: 376.1997 [1998].

BASIONYM: Epidendrum boothianum Lindl., Edward's Bot. Reg. 24: (Misc.) 5. 1838.

Epicladium boothianum (Lindl.) Small, Fl. Miami 56. 1913.

Hormidium boothianum (Lindl.) Brieger, Publ. Ci. Inst. Genét. Esc. Super. Agric. Luis de Queiroz 1: 21.1960.

Encyclia boothiana (Lind1.) Dressler, Brittonia 13: 264. 1961.

Type: Cuba: "Havannah," 1835, Sutton s.n. (holotype K-L).

Encyclia bidentata (Lindl.) Hágsater \& Soto Arenas, Orquídea (México) 13: 215. 1993.

Type: Mexico: Pav. s.n., México Herb. Pav., but likely collected in Cuba by Sessé \& Mociño (Hágsater, 1993; holotype BM).

Epicladium boothianum var. erythronioides (Small) Acuña, Bol. Estación Exp. Agron.
Santiago de las Vegas 60: 89.1939.

Prosthechea boothiana var. erythronioides (Small)

W.E.Higgins, North Amer. Nat. Orchid Jour.

5: 18.1999.

Type: United States of America: Florida, Key

Largo, A. H. Curtiss s.n. (holotype NY).

Specimens studied: HUJB!: Ferro et al. 4306; HOS!: Bocourt et al. 127; flowers in spirit: Bocourt et al. 051.

Prosthechea cochleata (L.) W.E.Higgins, Phytologia 82: 377.1997 [1998].

BASIONYM: Epidendrum cochleatum L., Sp. Pl. ed. 2: 1351. 1763.

Anacheilium cochleatum (L.) Hoffmans, Linnaea 16(Litt.): 229. 1842.

Encyclia cochleata (L.) Dressler, Brittonia 13: 264. 1961.

Type: Haiti: Plumier s.n. (Lectotype, Garay \& Sweet, 1974, p. 130, original illustration at P).

Epidendrum triandrum (Ames) House,

Muhlenbergia 1: 129. 1906.

Prosthechea cochleata var. triandra (Ames)

W.E.Higgins, North Amer. Nat. Orchid Jour. 4: 52.1998.

Type: United States of America: Florida, Ft. Lauderdale (lectotype Nir, 2000, p. 407).

Specimens studied: HAC!: Baker 4638; HUJB!: Ferro et al. 1742; HOS!: Agapito et al. 006; flowers in spirit: Pérez 068.

Prosthechea fragrans (Sw.) W.E.Higgins, Phytologia 82: 377.1997 [1998].

Basionym: Epidendrum fragrans Sw., Prodr. Veg. Ind. Occ. 123. 1788.

Anacheilium fragrans (Sw.) Acuña, Bol. Estación

Exp. Agron. Santiago de las Vegas 60: 86. 1939.

Encyclia fragrans (Sw.) Dressler, Brittonia 13:

264. 1961.

Hormidium fragrans (Sw.) Brieger, Publ. Ci. Inst.

Genét. Esc. Super. Agric. Luis de Queiroz 2: 69. 1961.

Type: Jamaica: O. Swartz s.n. (BM).

Specimens studied: HAJB!: Bisse et al. 22448; HOS!: flowers in spirit: Pérez \& Bocourt 046.

Prosthechea fuertesii (Cogn.) E.A.Christenson, Richardiana 8: 29. 2007.

BAsionym: Epidendrum fuertesii Cogn., in I.Urban, Symb. Antill. 7: 180. 1912. 
Type: Dominican Republic: Prov. Barahona, $M$. Fuertes 675 (holotype BR).

Specimens Studied: HAC!: Acuña et al. 19627; HOS!: Pérez \& Bocourt 161; flowers in spirit: Pérez \& Bocourt 092.

Prosthechea fuertesii is the Caribbean representative of the broadly distributed $P$. vespa complex. The Caribbean populations differ consistently from those of the continent primarily by the color of their flowers. The sepals and petals in the Caribbean plants lack the numerous reddish brown spots that are typical of the complex elsewhere and they may also differ vegetatively (Ackerman, 2014).

Prosthechea pygmaea (Hook.) W.E.Higgins, Phytologia 82: 380. 1997 [1998].

Basionym: Epidendrum pygmaeum Hook., J. Bot. 1: 49, t. 118. 1833.

Hormidium pygmaeum (Hook.) Benth. \& Hook.f. ex Hemsl., Biol. Cent.-Amer. Bot. 3: 218. 1883.

Encyclia pygmaea (Hook.) Dressler, Brittonia 13: 265. 1961.

Type: Brazil: Hort. Harrison, (holotype K-L).

Coelogyne triptera Brongn., in L.I.Duperrey, Voy. Monde 201. 1834.

Hormidium tripterum (Brongn.) Cogn., in Martius, Fl. Bras. 3(5): 29. 1898.

Encyclia triptera (Brongn.) Dressler \& G.E. Pollard, Phytologia 21: 438. 1971.

Type: Brazil: Île Sainte-Catherine, J. S. C. Dumont D'urville s.n. (holotype P).

Specimens STUdied: HUJB!: Urquiola et al. 5912; HOS!: Mújica 148; flowers in spirit: collector unknown 160.

\section{Pseudogoodyera Schlechter}

Pseudogoodyera wrightii (Rchb.f.) Schltr., Beih. Bot. Centralbl., Abt. 2, 37(2): 370. 1920. *

BASIONYM: Goodyera wrightii Rchb.f., Flora 27: 274. 1865.

Goodyera erythrosticta Griseb., Cat. Pl. Cub. 268. 1866.

Spiranthes wrightii (Rchb.f.) Schltr., in I.Urban, Symb. Antill. 7: 492. 1912.

Type: Cuba: 1860-1864, Wright 3297 (holotype W). SPECIMEN STUdied: HAC!: Alain et al. 4720.
Recently the authors were able to make perhaps the first color picture of this species with clearly open flowers, the opposite to what happens with the species in Mexico and Belize. It may be that Cuban and Central American populations belong to different species (G. Salazar, pers. comm. 2013).

\section{Psilochilus Barbosa Rodrigues}

Psilochilus macrophyllus (Lind1.) Ames, Orchidaceae 7: 45. 1922.

Basionym: Pogonia macrophylla Lindl., Ann. Mag. Nat. Hist., ser. 3, 1: 335. 1858.

Type: Cuba: C. Wright 615 (holotype K-L).

SPECIMEN STUdiEd: HAJB!: Alvarez et al. 57189.

\section{Sacoila Rafinesque}

Sacoila lanceolata (Aubl.) Garay, Bot. Mus. Leafl. 28: 352. 1982.

Basionym: Limodorum lanceolatum Aubl., Hist. Plant. Guian. Franc. 2: 821. 1775.

Stenorrhynchos lanceolatum (Aubl.) Richard ex Spreng., Syst. Veg. 3: 710. 1826.

Spiranthes lanceolata (Aubl.) León, Fl. Cub. 1: 358. 1946.

Type: Plumier s.n. (holotype, the original illustration $\mathrm{P}$ ).

Satyrium orchioides Sw., Prodr. Veg. Ind. Occ. 118. 1788.

Neottia orchidoides (Sw.) Willd., Spec. Pl. 4: 73. 1805.

Stenorrhynchos orchioides (Sw.) Lindl., Gen. Sp. Orchid. P1. 477. 1840.

Spiranthes orchioides (Sw.) A.Rich., in R.de la Sagra, Hist. Fis. Cuba Bot. 11: 252. 1850.

Gyrostachys orchioides (Sw.) Kuntze, Revis. Gen. P1.2: 664. 1891.

Type: Jamaica: Swartz s.n. (lectotype UPS, as "Type", Garay \& Sweet, 1974, p.75).

SPECIMENS STUdied: HAJB!: Bisse et al. 53677; HOS!: Elaine et al. 245.

Scaphyglottis Poeppig \& Endlicher

Scaphyglottis modesta (Rchb.f.) Schltr., Repert. Spec. Nov. Regni Veg. 23: 46. 1926.

Basionym: Tetragamestus modestus Rchb.f., Bonplandia 2: 21. 1854. 
Ponera modesta (Rchb.f.) Rchb.f., Linnaea 41: 85. 1876.

Type: Tropical America, Joss s.n. (holotype W). Ponera striolata Rchb.f., Linnaea 41: 39. 1876.

Scaphyglottis striolata (Rchb.f.) Correll, Bot. Mus. Leafl. 9: 148. 1941.

Type: Tropical America, von Hüttner (holotype $\mathrm{W})$.

Specimen STUdied: HAJB!: Bassler et al. 60741.

Scaphyglottis reflexa Lindl., Edward's Bot. Reg. 25 (Misc.): 20. 1839.

Reichenbachanthus reflexus (Lindl.) Porto \& Brade, Rodriguesia 1(2): 55. 1935.

Hexisea reflexa Rchb.f. ex Griseb., Fl. Brit. W. I. 623.1864.

Type: Guyana: "Obtained by Messrs. Loddiges from Demerara" Loddiges s.n. (K-L).

Reichenbachanthus emarginatus Garay, Bot. Mus. Leafl. 21: 255. 1967.

Type: Surinam: Mariepaston, Kegel 1422 (W).

SPECIMEN STUdied: HAC!: Alain 7345.

\section{SChiedeELla Schlechter}

Schiedeella amesiana Garay, Bot. Mus. Leafl. 28: 357. 1982; (avowed substitute for Spiranthes wrightii Ames, Orchidaceae 7: 131. 1922, non Spiranthes wrightii (Rchb.f.) Schltr., 1913).

Type: Cuba: Prov. Guantánamo, Monte Verde, Wright 1480 (holotype AMES).

SPECIMENS StUdied: We did not find any specimens in the reviewed Cuban herbaria.

\section{Spathoglottis Blume}

Spathoglottis plicata Blume, Bijdr. Fl. Ned. Ind. 401. 1825.

Type: Java: Blume s.n. (holotype L).

Bletia angustifolia Gaudich., Voy. Uranie 421. 1829.

Spathoglottis angustifolia (Gaudich.) Benth. \& Hook.f., Gen. Pl. 3: 512. 1883.

Type: Moluccas: Freycinet s.n. (holotype P).

SPeCimen STUdied: HOS!: flowers in spirit: Padilla 002 .

Introduced in Cuba from Asia as an ornamental plant and naturalized in the island.

\section{Specklinia Lindley}

Specklinia aristata (Hook.) Pridgeon \& M.W.Chase, Lindleyana 16: 256. 2001.

Basionym: Pleurothallis aristata Hook., Ann. Mag. Nat. Hist., ser. 1(2): 329. 1839.

Humboltia aristata (Hook.) Kuntze, Revis. Gen. P1. 2: 667. 1891.

Muscarella aristata (Hook.) Luer, Monogr. Syst. Bot. Missouri Bot. Gard. 105: 96. 2006.

Type: Guyana: near Demarara, introduced and flowered by Parker s.n. (lectotype [Stenzel, 2007, p.111], Annals and Magazine of Naural History, ser. 2, 11: t. 15. 1839).

Pleurothallis barberiana Rchb.f., Gard. Chron. 2: 6. 1881.

Humboltia barberiana (Rchb.f.) Kuntze, Revis. Gen. P1. 2: 667, 1891.

Type: J. Barber s.n. (holotype W).

Pleurothallis urbaniana Rchb.f., Ber. Deutsch. Bot. Ges. 3: 297. 1885.

Type: Puerto Rico: near Maricao, Indiera Fria, 3 Dec 1884, P. Sintenis 503 (holotype W).

SPecimens Studied: We did not find any specimens in the reviewed Cuban herbaria. Reported by Ackerman (2014) from Sierra Maestra, Santiago de Cuba province.

Specklinia brighamii (S.Watson) Pridgeon \& M.W.Chase, Lindleyana 16: 256. 2001.

Basionym: Pleurothallis brighamii S.Watson, Proc. Amer. Acad. Arts 23: 285. 1888.

Sarcinula brighamii (S.Watson) Luer, Monogr.

Syst. Bot. Missouri Bot. Gard. 105: 206. 2006.

Type: Guatemala: Alta Verapaz, forests of Chocón,

S. Watson s.n. (holotype AMES \#72461).

SPeCimen StUdied: HAJB!: Arias et al. 58740.

Specklinia corniculata (Sw.) Steud., Nomencl. Bot., ed. 2, 2: 431.1841.

Basionym: Epidendrum corniculatum Sw., Prodr. Veg. Ind. Occ. 123. 1788.

Dendrobium corniculatum (Sw.) Sw., Nova Acta Regiae Soc. Sci. Upsal. 6: 83. 1799.

Cymbidium corniculatum (Sw.) Spreng., Syst. Veg. 3: 722. 1826.

Pleurothallis corniculata (Sw.) Lindl., Edward's Bot. Reg. 28 (Misc.): 83. 1842.

Humboltia corniculata (Sw.) Kuntze, Revis. Gen. P1. 2: 667. 1891. 
Sarcinula corniculata (Sw.) Luer, Monogr. Syst.

Bot. Missouri Bot. Gard. 105: 209. 2006.

Type: Jamaica: O. Swartz s.n. (lectotype BM).

Pleurothallis nubigena Lindl., Ann. Mag. Nat.

Hist. ser. 3, 1: 326. 1858, as rubigena.

Type: Cuba orientali, C. Wright 657 (holotype K-L).

Specklinia pyrsodes (Rchb.f.) Pridgeon \& M.W.Chase, Lindleyana 16: 259. 2001.

Type: Mr. William Saunders s.n. (holotype W).

SPECIMENS STUDIED: HUJB!: Luis et al. 3564; HOS!: Bocourt et al. 154.

Specklinia grisebachiana (Cogn.) Luer, Monogr. Syst. Bot. Missouri Bot. Gard. 95: 260. 2004.

Basionym: Pleurothallis grisebachiana Cogn., in I.Urban, Symb. Antill. 6: 409. 1909.

Type: Cuba: Prov. Guantánamo, San André near Monte Verde, C. Wright 1503 (holotype GOET).

Pleurothallis blepharoglossa Luer, Lindleyana 14: 111. 1999.

Specklinia blepharoglossa (Luer) Luer, Monogr. Syst. Bot. Missouri Bot. Gard. 95: 259. 2004.

Type: Cuba: Prov. Holguín, Moa, camino a La

Melba, Arroyo Las Comadres, C. Luer et al. 18654 (holotype HAJB).

Specimens studied: HAJB!: Bisse et al. 53193; HAC!: Alain et al. 4674A; HUJB!: Urquiola et al. 5469.

Specklinia helenae (Fawc. \& Rendle) Pridgeon \& M.W.Chase, Lindleyana 16: 258. 2001.

Basionym: Pleurothallis helenae Fawc. \& Rendle, J. Bot. 47: 4. 1909.

Muscarella helenae (Fawc. \& Rendle) Luer, Monogr. Syst. Bot. Missouri Bot. Gard. 105: 105. 2006.

Type: Jamaica: Parish Portland, Mabess River, $W$. Harris s.n. (holotype BM).

SPECIMENS STUDIED: HAJB!: Dietrich 63418; HAC!: León et al. 7186.

Specklinia lichenicola (Griseb.) Pridgeon \& M.W.Chase, Lindleyana 16: 258. 2001. *

Basionym: Pleurothallis lichenicola Griseb., Cat. P1. Cub. 259. 1866.

Type: Cuba: Prov. Guantánamo, near Monte Verde, C. Wright 1507 (holotype W).

Pleurothallis gemina H.Stenzel, Lindleyana 16: 28.2001.
Type: Cuba: Holguín, Sierra de Nipe, Loma Mensura, Stenzel \& Matos 452 (HAJB!).

SPecimens STUdied: HAJB!: Bisse et al. 18631; HAC!: Alain et al. 7189.

Specklinia llamachoi (Luer) Luer, Monogr. Syst. Bot. Missouri Bot. Gard. 95: 261. 2004. *

BASIONym: Pleurothallis llamachoi Luer, Lindleyana 13: 146. 1998.

Muscarella llamachoi (Luer) Luer, Monogr. Syst. Bot. Missouri Bot. Gard. 105: 109. 2006.

Type: Cuba: Holguín, Mayarí, Sierra de Nipe, $C$. Luer et al. 18631 (holotype HAJB!).

SPECIMENS STUdied: HAJB!: Bisse et al. 52964; HAC!: Alain et al. 7867.

Specklinia longilabris (Lindl.) Luer, Monogr. Syst. Bot. Missouri Bot. Gard. 95: 261. 2004. *

Basionym: Pleurothallis longilabris Lindl., Ann. Mag. Nat. Hist., ser. 3, 1: 328. 1858.

Muscarella longilabris (Lindl.) Luer, Monogr. Syst. Bot. Missouri Bot. Gard. 105: 110. 2006. Type: Cuba: Oriente, $C$. Wright 651 in part (K-L). SPeCiMENS STUdied: HAJB!: Bisse et al. 53396; HAC!: Alain et al. 5463.

Specklinia mucronata (Lindl. ex Cogn.) Luer, Monogr. Syst. Bot. Missouri Bot. Gard. 95: 262. 2004.*

Basionym: Pleurothallis mucronata Lindl. ex Griseb., in I.Urban, Symb. Antill. 6: 424.1909.

Type: Cuba: Prov. Guantánamo, near Monte Verde, C. Wright 1504 (BR).

SPeCimENS STUdied: HAJB!: Alvarez et al. 57350-A; HAC!: Alain et al. 7195.

Specklinia obliquipetala (Acuña \& C.Schweinf.) Luer, Monogr. Syst. Bot. Missouri Bot. Gard. 95: 262. 2004. *

BAsionym: Pleurothallis obliquipetala Acuña \& C.Schweinf., Bot. Mus. Leafl. 6: 31938.

Trichosalpinx acunae (Acuña \& C.Schweinf.) Luer, Phytologia 54: 394. 1983.

Type: Cuba: Prov. Santiago de Cuba, Pico Turquino, Acuña 9540 (holotype AMES).

SPECIMEN STUdied: HAC!: Acuña 10567.

Specklinia schaferi (Ames) Luer, Monogr. Syst. Bot. Missouri Bot. Gard. 95: 263. 2004.

Basionym: Pleurothallis schaferi Ames, Orchidaceae 7: 119. 1922.

Type: Cuba: Prov. Holguín, Sierra de Nipe, near Woodfred, J. A. Schafer 3441 (lectotype AMES). 
Pleurothallis bipapularis Dod, Moscosoa 1: 52. 1976.

Specklinia bipapularis (Dod) Luer, Monogr. Syst. Bot. Missouri Bot. Gard. 95: 259. 2004.

Type: Bayaguana, República Dominicana, Prov. Monte Plata, La Lechuza, Pilancón, Bayaguana, Dod 504 (Holotype JSD; isotypes AMES, SEL, NY).

Pleurothallis haitiensis Dod, Moscosa 3: 113. 1984.

Specklinia haitiensis (Dod) Pridgeon \& M.W.Chase, Lindleyana 16: 258. 2001.

Type: Haiti: Massif de la Hotte, Les Cayes, Dod 890 (holotype JBSD; isotypes AMES, MO, NY, US).

Specimen Studied: HAJB!: Bisse et al. 49283.

Specklinia tribuloides (Sw.) Pridgeon \& M.W.Chase, Lindleyana 16: 259. 2001.

Basionym: Epidendrum tribuloides Sw., Prodr.

Veg. Ind. Occ. 123. 1788.

Dendrobium tribuloides (Sw.) Sw., Nova Acta Regiae Soc. Sci. Upsal. 6: 83. 1799.

Cymbidium tribuloides (Sw.) Spreng., Syst. Veg., ed. 16, 3: 721. 1826.

Pleurothallis tribuloides (Sw.) Lindl., Gen. Sp. Orchid. P1. 6. 1830.

Criptophoranthus tribuloides (Sw.) H.Dietr., Revista Jard. Bot. Nac. Univ. Habana 5: 48. 1984.

Tribulago tribuloides (Sw.) Luer, Monogr. Syst. Bot. Missouri Bot. Gard. 95: 265. 2004.

Type: Jamaica: O. Swartz s.n. (lectotype BM).

SPeCimens StUdied: HUJB!: Luis et al. 3111; HOS!: collector unknown 129; flowers in spirit: collector unknown 053.

Specklinia trichyphis (Rchb.f.) Luer, Monogr. Syst. Bot. Missouri Bot. Gard. 95: 264. 2004. *

Basionym: Pleurothallis trichyphis Rchb.f., Flora 48: 276. 1866.

Type: Cuba: 1860-1864, C. Wright 3345 (holotype $\mathrm{K})$.

SPecimen Studied: HAJB!: Bisse et al. 52961.

Specklinia wrightii (Rchb.f.) Luer, Monogr. Syst. Bot. Missouri Bot. Gard. 95: 265. 2004.*

Basionym: Pleurothallis wrightii Rchb.f., Flora 48: 276. 1865.

Type: Cuba: Prov. Guantánamo, near Monte Verde, C. Wright 1509 (W).
Pleurothallis richteri H.Dietr., Orchidee 35: 223. 1984.

Type: Cuba: Prov. Guantánamo: Baracoa, Vega de la Palma, around Río Duaba, Bisse et al. s.n. (holotype HAJB!).

Specimen StUdied: HAJB!: Alvarez et al. 56347.

\section{SPIRANTHES L. C. Richard}

Spiranthes torta (Thunb.) Garay \& H.R.Sweet, in R.A.Howard, Fl. Lesser Antilles, Orchid. (1): 77. 1974.

Basionym: Ophrys torta Thunb., Mus. Nat. Acad. Ups. 9: 136. 1791.

Type: Jamaica: Swartz s.n. (holotype UPS).

Neottia tortilis Sw., Kongl. Vetensk. Acad. Handl. 21: 226. 1800 .

Spiranthes tortilis (Sw.) L.C.Rich., Orch. Europ. Ann. 37. 1817

Type: Jamaica, Swartz s.n. (BM).

Satyrium spirale Sw., Prodr. Veg. Ind. Occ. 118. 1788.

Triorchis spiralis (Sw.) House, Amer. Midl. Naturalist 6: 206. 1920.

Type: Jamaica: Swartz s.n. (BM).

SPeCimens STUdied: HUJB!: Urquiola et al. 3377; HUCLV!: Expedición Botánica “J. Bisse” 2186; HOS!: Pérez et al. 089.

\section{Stelis Swartz}

Stelis antillensis Pridgeon \& M.W.Chase, Lindleyana 17: 98. 2002.

Pleurothallis domingensis Cogn., in I.Urban, Symb. Antill. 6: 402. 1909.

Stelis domingensis (Cogn.) Pridgeon \& M.W.Chase, Lindleyana 16: 262. 2001.

Crocodeilanthe domingensis (Cogn.) Luer, Monogr. Syst. Bot. Missouri Bot. Gard. 95: 256. 2004.

Type: Dominican Republic: Prov. La Vega, Valle Nuevo, Eggers 2176 (holotype BR).

SPecimen StUdied: HAJB!: Lippold 16133.

Stelis cubensis Schltr., in I.Urban, Symb. Antill. 9: 60. 1923.*

Type: Cuba: Prov. Santiago de Cuba, Sierra Maestra, Loma de Regino, near Pico Turquino, E. L. Ekman 5407 (holotype S).

SPECIMEN STUdied: HAC!: Acuña 15082. 
Stelis ekmanii Schltr., in I.Urban, Symb. Antill. 9: 60. 1923.*

Type: Cuba: Prov. Holguín, Sierra de Nipe, Loma Mensura, E. L. Ekman 3187 (holotype S).

SPecimen Studied: HAJB!: Bisse et al. 40293.

Stelis gelida (Lind1.) Pridgeon \& M.W.Chase, Lindleyana 16: 263. 2001.

Basionym: Pleurothallis gelida Lindl., Edward's Bot. Reg. 27 (Misc.): 91. 1841.

Humboltia gelida (Lindl.) Kuntze, Revis. Gen. Pl. 2: 667. 1891.

Specklinia gelida (Lindl.) Luer, Monogr. Syst. Bot. Missouri Bot. Gard. 95: 260. 2004.

Type: Jamaica: Loddiges s.n. (holotype K-L).

Pleurothallis univaginata Lindl., Ann. Mag. Nat. Hist., ser. 3, 1: 326. 1858.

Humboltia univaginata (Lindl.) Kuntze, Revis. Gen. Pl. 2: 668. 1891.

Type: Cuba: Prov. Guantánamo: near Monte Verde, 1856-57, C. Wright 656 (holotype K-L). Specimens studied: HAJB!: Arias et al. 60050; HAC!: Alain 7172; HOS!: Bocourt et al. 151; flowers in spirit: Bocourt et al. 083.

Stelis multirostris Pridgeon \& M.W.Chase, Lindleyana 17: 100. 2002.

BAsionym: Pleurothallis multirostris Rchb.f., Linnaea 41: 49. 1876.

Type: Jamaica: no collector indicated (holotype K). Pleurothallis oblongifolia Lindl., Bot. Mag. 2: 355. (1836) 1837.

Stelis oblongifolia (Lindl.) Pridgeon \& M.W.Chase, Lindleyana 16: 265. 2001, non Stelis oblongifolia Lindl. 1858.

Dracontia oblongifolia (Lindl.) Luer, Monogr. Syst. Bot. Missouri Bot. Gard. 95: 257. 2004.

Type: Jamaica: Loddiges s.n. (holotype K-L).

Epidendrum racemiflorum Sw., Prodr. Veg. Ind. Occ. 125. 1788.

Dendrobium racemiflorum (Sw.) Sw., Nova Acta Regiae Soc. Sci. Upsal. 6: 83. 1799.

Pleurothallis racemiflora Lindl. ex Hook., Exot. Fl. 2: t. 123. 1825 (1824).

Type: Jamaica, Swartz s.n. (lectotype BM).

Pleurothallis tricostata Cogn., in I.Urban, Symb. Antill. 7: 175. 1912.

Type: Dominican Republic: Prov. La Vega, Constanza: epiphytic in forest near Constanza,
H. von Türckheim 3481 (holotype B, destroyed).

Specimens Studied: HAJB!: Bisse 42262; HOS!: Pérez \& Bocourt 130; flowers in spirit: Pérez \& Bocourt 055.

\section{Stenorrhynchos L. C. Richard ex Sprengel}

Stenorrhynchos speciosum (Jacq.) L.C.Rich. ex Spreng., Mem. Mus. Hist. Nat. 4: 59. 1818.

Basionym: Neottia speciosa Jacq., Collectanea 3: 174. 1791.

Serapias speciosa (Jacq.) Gmel., Syst. Nat., ed. 13, 59. 1791.

Ibidium speciosum (Jacq.) Salisb., Trans. Hort. Soc. London 1: 291. 1812.

Spiranthes speciosa (Jacq.) A.Rich., in R.de la Sagra, Hist. Fis. Cuba Bot. 11: 252. 1850.

Gyrostachys speciosa (Jacq.) Kuntze, Revis. Gen. P1. 664. 1891.

Type: Jacquin s.n. (holotype W).

Specimen Studied: HUJB!: Urquiola et al. 1045.

\section{SudAmerlycaste Archila}

Sudamerlycaste pegueroi Archila, Moscosoa 17: 23. 2011.

Type: Rep. Dominicana: Cordillera septentrional, Prov. Hermanas Mirabal, Municipio de Salcedo, La Jíbara, Holotype JBSD: Rodríguez et al. 463.

Specimen Studied: HAC!: Alain 18640.

Previously assumed to be S. barringtoniae (a species which Ackerman consider to be endemic to Jamaica), the most obvious characteristic that distinguishes the two species is the length of the scape (Ackerman, 2014).

\section{Tetramicra Lindley}

Tetramicra ekmanii Mansf., Ark. Bot. 20A (15): 17. 1926.

Type: Haiti: Dept. d'Artibonite, Ennery, E. L. Ekman H 2474 (holotype S?; K?).

Tetramicra montecristensis H.Dietr., Orchidee 35: 192. 1984.

Type: Cuba: Prov. Guantánamo, Mun. Yateras, Pinar de Monte Cristi, J. Bisse et al. (holotype HAJB!). 
Tetramicra malpighiarum J.A.Hern. \& M.A.Díaz, Harvard Pap. Bot. 5: 189. 2000.*

Type: Cuba: Prov. Granma, El Guafe, Cabo Cruz, Jun 1996, J. A. Hernández 41199 (holotype HAC!).

Tetramicra parviflora Lindl. ex Griseb., Fl. Brit. W. I. 622.1864.

Type: Jamaica: MacFayden s.n. (holotype K).

Basionym: Bletia parviflora Rchb.f., Ann. Bot. Syst. 6: 439. 1862.

Type: Jamaica, D. Morris s.n. (holotype K).

Bletia schoeniana Rchb.f., Ann. Bot. Syst. 6: 439. 1862.

Tetramicra schoeniana (Rchb.f.) Rolfe, Gard. Chron., ser. 3, 6: 623. 1889.

Type: Dominican Republic, Schomburgk 36 (holotype K-L).

Tetramicra urbaniana Cogn., in I.Urban, Symb. Antill. 6: 551. 1910.

Type: Bahamas: New Providence, Curtiss 145 (holotype BM).

Specimens studied: HAJB!: Bisse 21562; HUJB!: Urquiola et al. 6152.

Tetramicra riparia Á.Vale, Sánchez-Abad \& L.Navarro, Syst. Bot. 37(4): 883-892. 2012.*

Type: Cuba: Guantánamo: Baracoa, orillas del río Báez, Mina Amores. Febrero/2010. Á. Vale, N.L. Sánchez-Abad \& J.A. Rodríguez, AVG 2010-04 (Holotype: HAC, Isotypes: BSC, M, SANT).

Tetramicra simplex Ames, Schedul. Orchid. 6: 77. 1923.*

Type: Cuba: Mun. Isla de la Juventud, near Los Indios, O. E. Jennings 644 (NY).

Specimen studied: HAC!: Killip 7459.

Tetramicra tenera Griseb. ex Benth., J. Linn. Soc., Botany 18: 314. 1881.*

Basionym: Bletia tenera A.Rich., in R.de la Sagra, Hist. Fis. Cuba Bot. 11: 242, t. 83. 1850.

Tetramicra eulophiae (Rchb.f.) Rchb.f. ex Griseb., Cat. Pl. Cub. 264. 1866.

Type: Cuba, C. Wright 3334 (holotype W).

Tetramicra erosa Carabia, Mem. Soc. Cub. Hist. Nat. "Felipe Poey" 7: 143. 1943.

Type: Cuba: Prov. Santiago de Cuba, Sagra s.n. (holotype P).

SPECIMENS STUdIED: HAJB!: Moreno 68455; HAC!: León 7454; HUJB!: Urquiola et al. 524; HOS!: Mújica \& Elaine 204; flowers in spirit: collector unknown 109.

\section{Tolumnia Rafinesque}

Tolumnia acunae (M.A.Díaz) Nir, Orchid. Antill. 373. 2000.*

Tolumnia tuerckheimii subsp. acunae M.A.Díaz, Revista Jard. Bot. Nac. Univ. Habana 8(2): 18. 1987.

Type: Cuba: Prov. Guantánamo, Imías, Sierra de Imías, camino entre Tres Piedras y la María, $J$. Bisse et al. (holotype HAJB!).

Tolumnia calochila (Cogn.) Braem, Orchidee 37: 58. 1986.

BAsionym: Oncidium calochilum Cogn., in I.Urban, Symb. Antill. 6: 660. 1910.

Type: Dominican Republic: Prov. Santiago, Llano Rafael, Eggers 1927 (holotype BR).

Specimens Studied: HAJB!: Gutiérrez et al. 68166; HUCLV!: Sotomayor 3868.

Tolumnia guibertiana (A.Rich.) Braem, Orchidee 37: 58. 1986.*

BAsIonym: Oncidium guibertianum A.Rich., in R.de la Sagra, Hist. Fis. Cuba Bot. 11: 244. 1850.

Tolumnia lemoniana subsp. guibertiana (A.Rich.)

Braem, Schlechteriana 1: 40. 1990.

Type: Cuba, Guibert s.n. (holotype P).

Specimens studied: HAJB!: Bisse et al. 46435; HOS!: Pérez et al 099; flowers in spirit: Pérez \& Bocourt 003.

Tolumnia gundlachii (C.Wright ex Griseb.) N.H.Williams \& Ackerman, Orchids (West Palm Beach) 76: 857. 2007.

Basionym: Oncidium gundlachii C.Wright ex Griseb., Cat. Pl. Cub. 266. 1866.

Antillanorchis gundlachii (C.Wright ex Griseb.)

Garay, Bradea 1: 423. 1974.

Type: Cuba: near Hanábana, Gundlach s.n. (holotype GOET).

Rodrigueziopsis antillensis Withner, Amer. Orchid Soc. Bull. 40: 876. 1971.

Type: Dominican Republic: Prov. Pedernales, Baoruco Mountains, Hoya de Pelempito, E of Aceitillar, A. H. Liogier 16724 (holotype NY). SPECimens STUdied: We did not find any specimens in the reviewed Cuban herbaria.

The type comes from Hanábana, locality that Sauget \& Barbier (1946) attribute to Matanzas province. In that province we can find with that name only a river 
that is in lowlands. Ackerman (pers. comm. 2010) suspects that this is an erroneous record because in the Domincan Republic this is a high elevation species, unlike Matanzas.

Tolumnia hawkesiana (Moir) Braem, Orchidee 37: 59. 1986. *

BAsionym: Oncidium hawkesianum Moir, Phytologia 15: 7. 1967.

Type: Cuba: Prov. Holguín, northern coast, Moir s.n. (holotype AMES).

SPECIMENS Studied: We did not find any specimens in the reviewed Cuban herbaria. This species is based on a plant collected from somewhere along the north coast of the old Oriente Province, presumably the current Province of Holguín (Ackerman, 2014).

Tolumnia lemoniana (Lind1.) Braem, Orchidee 37:

58. 1986. *

Basionym: Oncidium lemonianum Lindl., Bot. Reg. 21: t. 1789. 1835.

Type: Cuba, imported by Capt. Sutton and grown by Sir Charles Lemon (holotype original painting of t. 1789, K-L).

SPECIMENS STUDIED: HOS!: Pérez et al. 123; flowers in spirit: Pérez et al. 043.

Tolumnia lucayana (Nash) Braem, Orchidee 37: 58. 1986.

BASIONYM: Oncidium lucayanum Nash, in N.L.Britton \& C.F.Millspaugh, Bahama Fl. 98. 1920.

Type: Bahamas: Andros, Fresh Creek, J. I. and $A$. R. Northrop 647 (holotype NY).

Oncidium lyratum Withner, Amer. Orchid Soc. Bull.36: 400. 1967.

Tolumnia lyrata (Withner) Braem, Orchidee 37: 58. 1986.

Type: Cuba: Isle of Pines, W. Stimson s.n. (holotype AMES).

Oncidium moirianum Osment, Flor. Orch. 15: 147. 1972.

Tolumnia moiriana (Osment) Braem, Orchidee 37: 58. 1986.

Type: Cuba: Prov. Guantánamo, US Navy Base, W. W. G. Moir s.n. (holotype AMES).

Specimens studied: We did not find any specimens in the reviewed Cuban herbaria.

Tolumnia sylvestris (Lindl.) Braem, Orchidee 37: 59. 1986.*
Basionym: Oncidium sylvestre Lindl., Ann. Mag. Nat. Hist., ser. 3, 1: 332. 1858.

Oncidium variegatum subsp. sylvestre (Lindl.) Withner, Orchid Digest 44: 93. 1980.

Type: Cuba: "Oriente", 1856-1857, C. Wright 670 (holotype K-L).

SPecimen STUdied: HOS!: Cabrera 013.

Tolumnia usneoides (Lindl.) Braem, Orchidee 37: 59. 1986.*

Basionym: Oncidium usneoides Lindl., Ann. Mag.

Nat. Hist., ser. 3, 1: 33. 1858.

Type: Cuba: Prov. Guantánamo, Monte Verde, $C$. Wright 669 (holotype K-L).

SPecimens StUdied: We did not find any specimens in the revised Cuban herbaria.

Tolumnia variegata (Sw.) Braem, Orchidee 37: 59. 1986.

Basionym: Epidendrum variegatum Sw., Prodr. Veg. Ind. Occ. 122. 1788.

Cymbidium variegatum (Sw.) Sw., Nova Acta Regiae Soc. Sci. Upsal. 6: 74. 1799.

Oncidium variegatum (Sw.) Sw., Kongl. Vetensk. Acad. Handl. 21: 240. 1800.

Type: Haiti, "Santo Domingo": Swartz s.n. (BM). Epidendrum carinatum Vahl, in H.West, Bidr. Beskr. Ste Croix 303. 1793.

Type: United States Virgin Islands: St. Croix (holotype ?).

Oncidium velutinum Lindl. \& Paxton, in Paxton's Flower Garden 1: 166. 1851.

Tolumnia velutina (Lindl. \& Paxton) Braem, Orchidee 37: 58. 1986.

Type: Cuba: Santiago de Cuba, 1844, J. Linden 1759 (holotype K-L).

Oncidium leiboldii Rchb.f., in W.G.Walpers, Ann. Bot. Syst. 6: 718. 1863.

Tolumnia leiboldii (Rchb.f.) Braem, Orchidee 37: 58. 1986.

Type: Cuba: Wright $668(\mathrm{~W})$.

Oncidium caymanense Moir, Phytologia 17: 427. 1968.

Tolumnia caymanensis (Moir) Braem, Orchidee 37: 58. 1986.

Type: Cayman Islands: Grand Cayman, Moir s.n. (holotype AMES).

Tolumnia borinquinensis Sauleda \& Ragan, Orchid Digest 60: 183. 1996. 
Type: Puerto Rico, Lago Tortuguero, 2.3 km NE of Manatí, Sauleda et al. 6835 (holotype NY). Specimens StUdied: HUJB!: Urquiola 538; HOS!: Martinez et al. 121.

Trichocentrum Poeppig \& Endlicher

Trichocentrum undulatum (Sw.) Ackerman \& M.W.Chase, Lindleyana 16: 225. 2001.

Basionym: Epidendrum undulatum Sw., Prodr. Veg. Ind. Occ. 122. 1788.

Cymbidium undulatum (Sw.) Sw., Nova Acta Regiae Soc. Sci. Upsal. 6: 74. 1799.

Oncidium undulatum (Sw.) Salisb., Trans. Hort. Soc. London 1: 295. 1812.

Type: Jamaica, Swartz s.n. (S).

Epidendrum maculatum Aubl., Hist. Plant. Guian.

Franc. 2: 825. 1775.

Oncidium maculatum (Aubl.) Urb., Repert. Spec. Nov. Regni Veg. 15: 306. 1918.

Lophiaris maculata (Aubl.) Ackerman, Lindleyana 15: 89-95. 2000.

Trichocentrum maculatum (Aubl.) M.W.Chase \& N.H.Wms., Lindleyana 16: 218-219. 2001.

Type: Plumier, Plantarum Americanarum t. 178, fig. 2. 1758 (holotype. the original Burmann plate, reproduction).

Specimens studied: HAJB!: Bisse et al. 15060; HOS!: Mújica \& Elaine 194; flowers in spirit: Martínez 002.

\section{TrichopiLia Lindley}

Trichopilia fragrans (Lindl.) Rchb.f., Hamburger Garten-Blumenzeitung 14: 229. 1858.

Basionym: Pilumna fragrans Lindl., Edward's Bot. Reg. 30 (Misc.): 74. 1844.

Type: Colombia: near Popayan, Hartweg s.n. (holotype K-L).

Trichopilia backhousiana Rchb.f., Gard. Chron. 1: 816. 1876.

Type: New Grenada, Backhouse s.n. (holotype W). SpeCimens studied: HAJB!: Alvarez 64023; HAC!: Acuña 10066.

Trichopilia subulata (Sw.) Rchb.f., Flora 48: 278. 1865.

Basionym: Epidendrum subulatum Sw., Prodr. Veg. Ind. Occ. 123. 1788.
Cymbidium subulatum (Sw.) Sw., Nova Acta Regiae Soc. Sci. Upsal. 4: 73. 1799.

Leucohyle subulata (Sw.) Schltr., Orchideen Beschreib. Kult. Zucht. 469. 1914.

Type: Jamaica: Swartz s.n. (S).

Trichopilia jamaicensis Fawc. \& Rendle, J. Bot. 48: 107. 1910.

Leucohyle jamaicensis (Fawc. \& Rendle) Schltr., Orchideen Beschreib. Kult. Zucht. 469. 1914.

Type: Jamaica: Parish St. Thomas, Mansfield, near Bath, Harris 7697 (holotype BM).

Specimen STUdied: HAC!: Acuña 9795.

\section{Trichosalpinx Luer}

Trichosalpinx dura (Lindl.) Luer, Phytologia 54: 395. 1983.

Basionym: Pleurothallis dura Lindl., Fol. Orchid. 9: 32.1859.

Humboltia dura (Lindl.) Kuntze, Revis. Gen. Pl. 2: 667. 1891.

Type: Ecuador: forests of the Andes, W. Jameson s.n. (holotype K).

Pleurothallis foliata Griseb., F1. Brit. W. I. 610. 1864.

Humboltia foliata (Griseb.) Kuntze, Revis. Gen. P1. 2: 667.1891.

Trichosalpinx foliata (Griseb.) Luer, Phytologia 54: 395. 1983.

Type: Jamaica: 1857, Mr. Wilson 160 (lectotype $\mathrm{K})$.

Specimens STUdied: HAJB!: Alain et al. 5452; HOS!: Bocourt et al. 163.

Trichosalpinx memor (Rchb.f.) Luer, Phytologia 54: 396. 1983.

BAsionym: Pleurothallis memor Rchb.f., Bonplandia 4: 330. 1856.

Humboltia memor (Rchb.f.) Kuntze, Revis. Gen. P1. 2: 668. 1891.

Type: Colombia?, without collection data, Forkel s.n. (holotype W).

SPECIMENS STUdied: We did not find any specimens in the reviewed Cuban herbaria. Reported for Guantánamo (Macizo de Sagua-Baracoa), Sancti Spíritus (Alturas de Trinidad), and Santiago de Cuba (Sierra Maestra) provinces (Ackerman, 2014). 


\section{Triphora Nuttall}

Triphora gentianoides (Sw.) Nutt. ex Ames \& Schltr., in O.Ames, Orchidaceae 7: 5. 1922.

BAsionym: Limodorum gentianoides Sw., Prodr. Veg. Ind. Occ. 119. 1788.

Arethusa gentianoides (Sw.) Sw., Fl. Ind. Occid. 3: 1436.1806.

Pogonia gentianoides (Sw.) Spreng., Syst. Veg. 3: 706. 1826.

Type: Jamaica: Swartz s.n. (lectotype BM).

Pogonia cubensis Rchb.f., Ned. Kruidk. Arch. 4: 322. 1859.

Triphora cubensis (Rchb.f.) Ames, Schedul. Orchid. 7: 35. 1924.

Type: Cuba: Poeppig s.n. (holotype W).

SPECIMEN STUdied: HAC!: Casas de Almeida 15561.

Triphora miserrima (Cogn.) Acuña, Bol. Estación Exp. Agron. Santiago de las Vegas 60: 18. 1938 [1939].

Basionym: Pogonia miserrima Cogn., in I.Urban, Symb. Antill. 6: 316. 1909.

Type: Cuba: Prov. Villa Clara, between La Magdalena and Cayamas, Baker 4943 (holotype ?).

SPeCimens studied: We did not find any specimens in the revised Cuban herbaria.

Triphora surinamensis (Lindl. ex Benth.) Britton, in N.L.Britton \& P.Wilson, Sci. Surv. Porto Rico \& Virg. Isl. 5(2): 184. 1924.

Basionym: Pogonia surinamensis Lindl. ex Benth., London J. Bot. 2: 674. 1843.

Type: Guyana: Berbice River, Christmas Catarats, Schomburgks.n. (holotype K).

Triphora duckei Schltr., Beih. Bot. Centralbl., Abt. 2, 42(2): 75.1925.

Type: Brazil: Amazonas, Lake Salgado, Río Trombetes, Feb 1918, A. Ducke 16987 (holotype B, destroyed).

SPECIMEN STUdIED: HAC: Llamacho 42100.

\section{Tropidia Lindley}

Tropidia polystachya (Sw.) Ames, Orchidaceae 2: 262. 1908 .

Basionym: Serapias polystachya Sw., Prodr. Veg. Ind. Occ. 119. 1788.

Neottia polystachya (Sw.) Sw., Fl. Ind. Occid. 3: 1415. 1806.

Stenorrhynchos polystachyon (Sw.) Spreng., Syst.
Veg. 3: 710. 1826.

Tomotris polystachya (Sw.) Raf., F1. Tellur. 2: 89. 1837.

Chloidia polystachya (Sw.) Rchb.f., in W.G.Walpers, Ann. Bot. Syst. 6: 644. 1863.

Corymborkis polystachya (Sw.) Kuntze, Revis. Gen. P1. 658. 1891.

Corymbis polystachya (Sw.) Benth. ex Fawc.,

Prov. List Ind. Nat. Flow. P1. Jam. 40. 1893.

Type: Jamaica, mountains of Clarendon, Swartz s.n. (lectotype BM).

Specimens STUdied: HUJB!: Luis et al. 4053; HOS!: Mújica \& Elaine 211.

\section{VANilla Plumier ex Miller}

Vanilla bakeri Schltr., Repert. Spec. Nov. Regni Veg. 8: 561 (1910). *

Type: Cuba: Cojimar, Havana province, Baker 5127 (holotype B, destroyed; isotypes HAC, NY).

The holotype of $V$. bakeri was destroyed at B; a duplicate is housed at HAC, but it has only fruits. Vanilla bakeri appears to be closely related to $V$. barbellata, from which it differs by the smaller flowers and a cluster of $1 \mathrm{~mm}$ long fleshy hairs on the midlobe of the labellum. Nir (2000) recorded the existence of an isotype at NY (flowers in spirit), from wich he made a drawing of the flower. Soto Arenas \& Cribb (2010) were unable to see flowering material of $V$. bakeri, and the lectotype they designated was a plant in fruit. They speculated that the original description was based on immature flowers so they regarded $V$. bakeri to be a synonym of $V$. barbellata. They were apparently unaware of the alcohol-preserved specimen at NY (Ackerman, 2014).

Vanilla barbellata Rchb.f., Flora 48: 274 (1865).

Type: Cuba: C. Wright 3352, (holotype K).

Vanilla articulata Northr., Mem. Torrey Bot. Club 12: 31 (1902).

Type: Bahamas: Andros, Deep Creek, Jun 1890, J. I. Northrop \& A. R. Northrop 545 (not located). SPECIMENS STUDIED: HAJB!: Bisse et al. 53822; HAC!: Acuña et al. 16205.

Vanilla bicolor Lindl., Edward's Bot. Reg. 24 (Misc.): 37 (1838).

Type: Guyana, Demerara, watercolor painting of Schomburgk ex Hort. Loddiges s.n. (lectotype $\mathrm{K}-\mathrm{L})$. 
Vanilla wrightii Rchb.f., Flora 48: 273 (1865).

Vanilla gratiosa Griseb., Cat. Pl. Cub. 267. 1866.

Type: Cuba: C. Wright 672 (holotype K).

Specimens studied: HUJB!: Urquiola et al. 6316, 6310-A \& 6310-B.

Vanilla claviculata (W.Wright) Sw., Nova Acta Regiae Soc. Sci. Upsal. 6: 66 (1799).

BASIONYM: Epidendrum claviculatum W.Wright, in London Med. J. 8(3): 254 (1787).

Type: Jamaica: Swartz s.n. (holotype BM).

Specimen Studied: HAC!: León 19346.

Vanilla dilloniana Correll, Amer. Orchid Soc. Bull.15(7): 331 (1946).

Type: United States of America: Florida, Dade County, Brickell Hammock, Humes s.n. (holotype AMES).

Specimens Studied: HAJB!: Berazaín 66142; HAC!: Cano \& Herrera 34798.

Vanilla mexicana Mill., Gard. Dict., ed. 8, No.1 (1768).

Type: Haiti, Plumier s. n. (holotype Plumier's original drawing at $\mathrm{P}$; reproduced in Plumier, Nov. Pl. Amer. Genera 25, pl. 28, 1703).

Epidendrum vanilla L., Sp. Pl., ed. 1, 2: 952 (1753). Vanilla aromatica Sw., Nova Acta Regiae Soc. Sci. Upsal. 6: 66 (1799).

Vanilla vanilla (L.) Huth, in Helios 11(9): 136 (1893).

Vanilla vanilla (L.) Britton, in Britton \& Wilson, Sci. Surv. Porto Rico and Virg. Isl. 5: 185 (1924).

Type: "Volubilis siliquosa plantaginis folio" (lectotype [Cribb in Cafferty \& Jarvis, 1999], p. 47, Catesby, Natural History of Carolina, Florida and the Bahama Islands 2: app. t. 7. 1747).

Vanilla inodora Schiede, Linnaea 4: 574 (1829).

Type: Mexico, Schiede \& Deppe s.n. (holotype BM).

Vanilla anaromatica Griseb., Fl. Brit. W. I. 638 (1864).

Type: Jamaica, Based upon Plumier, Ed. Burm., t. 188.

SPeCimENS STUdied: HUJB!: Urquiola et al. 5275; HOS!: Bocourt et al. 080.

Vanilla palmarum Salzmann ex Lindl., Gen. Spec. Orch. P1. 436. 1840. *
Type: Brazil: Bahia, Salzmann s.n. (holotype K). Vanilla savannarum Britt., Mem. Torr. Club. 6: 61. 1920.

Type: Cuba: near Camagüey, N. L. Britton et al. 13120 (holotype NY).

SPeCimENS STUdied: HAJB!: Dietrich et al. 66652; HAC!: Acuña 13492.

Vanilla phaeantha Rchb.f., Flora 48: 274 (1865).

Type: Cuba: San Cristóbal, C. Wright 3351(holotype K).

Vanilla planifolia var. macrantha Griseb., Cat. P1. Cub. 267 (1866).

Specimen studied: HAC!: Alain 7501.

Vanilla poitaei Rchb.f., Linnaea 41: 66 (1876).

Type: Haiti: "Santo Domingo" Poiteau s.n. (holotype W).

Vanilla eggersii Rolfe, J. Linn. Soc. Bot. 32: 472 (1896).

Type: Dominican Republic: "Llanos de Rafael, Santo Domingo", Eggers 1958 (holotype K).

Vanilla correllii Sauleda \& R.M.Adams, Brittonia 33(2): 192 (1981).

Type: Bahamas: Grand Bahama, Freeport

Hammock off Coral Road, Correll et al. (holotype NY).

Specimen Studied: HAC!: Borhidi et al. 35473.

Wullschlaegelia Reichenbach $\mathrm{f}$.

Wullschlaegelia aphylla (Sw.) Rchb.f., Bot. Zeitung 21: 131. 1863.

Basionym: Cranichis aphylla Sw., Prodr. Veg. Ind. Occ. 120. 1788.

Type: Jamaica: Mountains near Clarendon, Swartz s.n. (lectotype BM).

SPeCiMEn StUdied: HAC!: Acuña 13052.

\section{Xуцовium Lindley}

Xylobium palmifolium (Sw.) Fawc., Prov. List. Pl. Jamaica 39. 1893.

Basionym: Epidendrum palmifolium Sw., Prodr. Veg. Ind. Occ. 123. 1788.

Dendrobium palmifolium (Sw.) Sw., Nova Acta Regiae Soc. Sci. Upsal. 6: 82. 1799.

Colax palmifolius (Sw.) Lindl. ex Spreng., Syst. Veg. 3: 727. 1826.

Maxillaria palmifolia (Sw.) Lindl., Gen. Sp. 
Orchid. P1. 148. 1832.

Type: Jamaica: Swartz s.n. (lectotype BM).

Maxillaria decolor Lindl., Edward's Bot. Reg. 18:

t. 1549.1832.

Xylobium decolor (Lindl.) G.Nicholson, Illust.

Dict. Gard. 4: 225. 1887.

Type: (holotype K-L, without data).

SPECIMEN STUdied: HAC!: Alain 15165.

According to Ackerman (2014) the Cuban specimens he observed annotated as $X$. pallidiflorum actually correspond to $X$. palmifolium.

\section{Zeuxine Lindley}

Zeuxine strateumatica (L.) Schltr., Bot. Jharb. Syst. 45: 394. 1911.

Basionym: Orchis strateumatica L., Sp. Pl. 943. 1753.

Neottia strateumatica (L.) R.Br., Prodr. Fl. Nov. Holl. 319. 1810.

Spiranthes strateumatica (L.) Lindl., Bot. Reg. 10: 823. 1824

Adenostylis strateumatica (L.) Ames, Orchidaceae 2: 58.1908.
Type: Herb. P. Hermann 2: 35, No. 319 (lectotype $\mathrm{BM})$.

Specimens studied: HAJB!: Alvarez et al. 43502; HOS!: Bocourt 235; flowers in spirit: collector unknown 162.

\section{ZOOTROPHION Luer}

Zootrophion atropurpureum (Lindl.) Luer, Selbyana 7: 80. 1982.

BASIONYM: Specklinia atropurpurea Lindl., Edward's Bot. Reg. 21, t. 1797. 1835.

Pleurothallis atropurpurea (Lindl.) Lindl., Edward's Bot. Reg. 28 (Misc.): 81. 1842.

Cryptophoranthus atropurpureus (Lindl.) Rolfe, Gard. Chron., ser. 3, 2: 693. 1887.

Type: Jamaica: cultivated at the Liverpool Botanical Garden (holotype K).

Masdevallia fenestrata Lindl. ex Hook., Bot. Mag. 71: t. 4164. 1845.

Type: Jamaica: Purdie s.n. (lectotype, Stenzel, 2007, p. 48, K).

SPecimens STUdied: HAJB!: Bisse et al. 39455; HAC!: Alain \& Acuña 7556.

\section{EXCLUDED SPECIES}

Cranichis wageneri Rchb.f., Linnaea 41: 19. 1876.

Recorded by Nir (2000) without specific location. The origin of this specimen is unknown, and its presence in Cuba cannot be confirmed (Ackerman, 2014).

Encyclia guanahacabibensis Sauleda \& Esperon, New World Orchid. Nomencl. Notes 7: 2. 2013 [30 Jun 2013].

Sauleda and Esperón (2013) describe Encyclia guanahacabibensis based on a plant cultivated by William Osment allegedly collected in the Guanahacabibes Peninsula, Cuba, in the 1950s. Although we do not definitively deny the presence of this species in Guanahacabibes, it seems improbable that it had escaped collection until Osment came along, and continues to do so after, especially considering that this is a well-documented area. We prefer to be conservative and exclude the species from the list.

Epidendrum vincentinum Lindl., J. Bot. (Hooker) 3: 88. 1840.
Cuba: Prov. Santiago de Cuba; Cordillera de la Gran Piedra. Known from Cuba by only a single collection of C. Wright at K (!). We don't know about the presence of this species in Cuba.

Epidendrum verrucosum Sw., Nova Acta Regiae Soc. Sci. Upsal 6: 68. 1799.

Acuña's (1939) report of E. verrucosum in Cuba is not yet substantiated.

Leochilus scriptus (Scheidweiler) Rchb.f., Xenia Orch.1(1): 15. 1854.

The presence of this species on the island is questionable; however, a Cuban herbarium specimen (Bohmhoff s.n., AMES) has been recorded for La Soledad, near Santa Clara in 1903. Mark Chase (1986) quoted the observation of two sterile specimens of this species that differ vegetatively from $L$. labiatus. In 1946, León recorded it for Cuba based solely on a specimen deposited in AMES, possibly the same mentioned before. The authors have been unable to locate any specimen of this species in the reviewed 
Cuban herbaria. Ackerman (2014) mentions that the specimens he observed were sterile and Chase's description was based on material collected outside of the Greater Antilles.

Myrmecophila thomsoniana var. minor (Strachan ex Fawc.) Dressler, Orquideología 22: 230. 2003.

Different authors recorded this species in Cuba, including Ackerman (2014). We suspect that the specimens observed may be plants that escaped cultivation and later naturalized, although this has yet to be confirmed.

Vanilla planifolia Andrews, Botanist's Repository 8: t. 538. 1808.

Ackerman cites this species for Cuba guided by the report of E. Mújica, (pers. comm. 2005). This report was an erroneous identification of $V$. phaeantha.
Final considerations. It is conceivable that new species and natural hybrids will eventually be collected and described, especially within the highly diverse genera Broughtonia, Encyclia, Lepanthes, and Pleurothallis.

Recently, colonization by Acampe rigida (Buch.Ham. ex Sm.) P.F.Hunt, Cymbidium sp., Dendrobium crumenatum Sw., and Papilionanthe teres (Roxb.) Schltr., was observed in close proximity to the Soroa Orchid Botanical Garden, suggesting that these species are capable of escaping cultivation without human assistance (Bocourt, pers. comm. 2015). Thus, it is plausible that these orchids will eventually become naturalized, adding to the total number.

According to Nir (2000), five additional orchid species are present on the neighboring islands of Jamaica and the Hispaniola that have yet to be reported in Cuba: Cyclopogon bicolor (Ker-Gawl.) Schltr., Liparis cardiophylla Ames, Pseudocentrum minus (Benth.) Hook., Tetramicra pratensis (Rchb.f.) Rolfe and Vanilla pompona Schiede. Due to the close proximity of these islands to Cuban archipelago, it would not be surprising to eventually encounter these species in Cuba
AcKnowledgements. We sincerely thank our Cuban colleagues that offered their time, valuable information and excellent feedback: José L. Bocourt and Esther Liliam Santa Cruz Cabrera from the Soroa Orchid Botanical Garden, Maité Serguera from the Ferns Botanical Garden of Santiago de Cuba and Juan Miguel Díaz from the Institute of Meteorology of Pinar del Río. Also, to Alelí Morales, Curator of the collection of the section Orchidaceae at HAJB, Tomi González from the HAC and Enrique González Pendás from HUJB for the facilities granted for the revision of those herbaria and to our colleagues Aldo Luis Ramos, senior environmental manager from Ecovida, Gilberto Barrera, from the Museum of Natural History and my cousin and namesake Ernesto Mujica, from New York, for their revision and translations of this report. Without their help, we would not have been able to complete this work.

\section{LitERATURE CITED}

Ackerman, J. D. (2012). Orchidaceae. In: G. R. Proctor (Ed.), Flora of the Cayman Islands, 2nd edition (pp. 187-

214). Chicago: University of Chicago Press.

Ackerman, J. D. (2014). The orchid flora of the Greater Antilles. New York: The New York Botanical Garden Press.

Acuña Galé, J. (1938). Catálogo descriptivo de las orquídeas cubanas. Boletín de la Estación Agronómica de Santiago de las Vegas, 60, 1-221.

Blanco, M. (2002). Notes o the natural history of Cyclopogon obliquus (Orchidaceae: Spiranthinae) in Costa Rica.

Lankesteriana, 5, 3-8.

Chase, M. W. (1986). A Monograph of Leochilus (Orchidaceae). Systematic Botany Monographs, 14, 1-97.

Chase, M. W., Cameron, K. M., Feudenstein, J. V., Pridgeon, A. M., Salazar, G., Van den Berg, C. \& Schuiteman, A.

(2015). An updated classification of Orchidaceae. Botanical Journal of the Linnean Society, 177, 151-174.

Cribb, P. J. \& Ormerod, P. (1999). Notes on the Orchidaceae from the Pacific Islands. Kew Bulletin, 55, 231-236.

Díaz, M. A. (1988). Las orquídeas nativas de Cuba. La Habana: Editorial Científico Téenica. 
Dietrich, H. (1984). Listado de especies de orquídeas cubanas y su distribución en la isla. Revista del Jardín Botánico Nacional de La Habana, 5(1), 32-51.

Dietrich, H. \& Stenzel, H. (2007). Flora de la República de Cuba, Orchidaceae I-II. Liechsteinstein: A.R. Gantner Verlag. Fawcett, W. \& Rendle, A. B. (1910). Flora of Jamaica. Vol. 1, Orchidaceae. London: British Museum.

Garay, L. A. (1977). Systematic of the Pgysurinae in the New World. Bradea, 2, 191-208.

Garay, L. A. (1982). A generic revision of the Spiranthinae. Botanical Museum Leaflets (Harvard University), 28, 277-425.

Gloudon, A. \& Tobisch, C. (1995). Orchids of Jamaica. Kingston: The Press University of The West Indies.

León, H. (1946). Orquídeas. In: J. S. H. León \& J. S. Sauget (Eds.), Flora de Cuba. Vol.1. La Habana: Museo de Historia Natural del Colegio La Salle.

Llamacho Olmo, J. A. \& Larramendi J. A. (2005). Las orquídeas de Cuba. España: Greta Editores.

Martos, F., Johnson, S. D., Peter, C. I. \& Bytebier, B. (2014). A molecular phylogeny reveals paraphyly of the large genus Eulophia (Orchidaceae): a case for the reinstatement of Orthochilus. Taxon, 63, 9-23.

Meneguzzo, T. E. C. (2012). Mudancas nomenclaturais em Goodyerinae do Novo Mundo (Orchidaceae). Orquidário, 26, 86-91.

Mújica, B. E., Pérez, R., López Trabanco, P. J., Bocourt, J. L. \& Ramos, T. (2000). Los géneros de orquídeas cubanas. La Habana: Editorial Félix Varela.

Nir, M. A. (2000). Orchidaceae Antillanae. New York: DAG Media Publishing Inc.

de la Sagra, R. D. (1845). Historia Física Política y Natural de la isla de Cuba. Segunda parte. Historia Natural. Tomo 10.

Botánica. Paris: Arthus Bertrand.

Ormerod, P. (2013). Studies of Neotropical Goodyerinae (Orchidaceae) 5. Harvard Papers in Botany, 18, 51-60.

Sauget y Barbier, S.J. (1946). Flora de Cuba, Vol. 1: Gimnospermas, Monocotiledoneas. Contrib. Ocac. Mus. Hist. Nat. Col. La Salle 8. Vedado, La Habana.

Sauleda, R. P. \& Esperón, P. (2013). A new species of Encyclia Hooker from the Guanahacabibes península of western Cuba. New World Orchidaceae. Nomenclatural Notes, 7, 1-15.

Soto Arenas, M. A. \& Cribb, P. (2010). A new infrageneric classification and sinopsis of the genus Vanilla Plum. ex Mill. (Orchidaceae: Vanillinae). Lankesteriana, 9(3), 355-398.

Stenzel, H. (2007). Pleurothallidinae, 1. In: H. Dietrich \& H. Stenzel (eds.). Flora de la Republica de Cuba. Series A. Vol. 15: Orchidaceae. Königstein, Koeltz Scientific Books.

Withner, C. L. (1996). The Bahamian and the Caribbean Species. The Cattleyas and their relatives. Oregon: Timber Press. 
